\title{
Heegaard Floer homology as morphism spaces
}

\author{
Robert Lipshitz, ${ }^{1}$ Peter S. Ozsváth ${ }^{2}$ and Dylan P. Thurston ${ }^{3}$
}

\begin{abstract}
In this paper we prove another pairing theorem for bordered Floer homology. Unlike the original pairing theorem, this one is stated in terms of homomorphisms, not tensor products. The present formulation is closer in spirit to the usual TQFT framework, and allows a more direct comparison with Fukaya-categorical constructions. The result also leads to various dualities in bordered Floer homology.
\end{abstract}

Mathematics Subject Classification (2010). Primary 52R58; Secondary 53D40.

Keywords. Heegaard Floer homology, topological quantum field theories, bordered Floer homology, Hochschild homology.

\section{Contents}

1 Introduction . . . . . . . . . . . . . . . . . 382

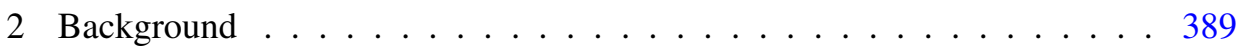

$3 \alpha$ - $\beta$-bordered Heegaard diagrams . . . . . . . . . . . . . . . . . . 397

4 An interpolating piece . . . . . . . . . . . . . . . . . 411

5 Consequences ....................... . 417

6 Gradings . . . . . . . . . . . . . . . . . . 427

7 Examples ......................... . . 428

8 Relation to Koszul duality . . . . . . . . . . . . . . . . . . 435

A User's guide to orientation conventions . . . . . . . . . . . . . . 446

References ....................... . . 448

\footnotetext{
${ }^{1}$ Robert Lipshitz was supported by an NSF Mathematical Sciences Postdoctoral Fellowship, NSF Grant number DMS-0905796 and a Sloan Research Fellowship.

${ }^{2}$ Peter S. Ozsváth was supported by NSF grant number DMS-0804121 and a Guggenheim Fellowship.

${ }^{3}$ Dylan P. Thurston was supported by a Sloan Research Fellowship.
} 


\section{Introduction}

In [9], we introduced extensions of the Heegaard Floer homology group $\widehat{\mathrm{HF}}(Y)$ (with coefficients in $\mathbb{F}_{2}=\mathbb{Z} / 2 \mathbb{Z}$ ) to 3-manifolds with boundary. To a surface $F$, together with a handle decomposition $\mathcal{Z}$ of $F$ and a little extra data (in the form of a basepoint), we associated a differential graded algebra $\mathcal{A}(\mathcal{Z})$. To a 3-manifold $Y$ with boundary parameterized by $\mathcal{Z}$, we associated a right $\mathcal{A}_{\infty}$-module $\widehat{\mathrm{CFA}}(Y)$ over $\mathcal{A}(Z)$ and a left differential graded module $\widehat{\mathrm{CFD}}(Y)$ over $\mathcal{A}(-Z)$ (where - denotes orientation reversal), each of which is well-defined up to homotopy equivalence in the corresponding category. These relate to the closed invariants via the pairing theorem, which states that if $Y_{1}$ and $Y_{2}$ are 3-manifolds with boundaries parameterized by $F$ and $-F$ respectively then

$$
\widehat{\mathrm{HF}}\left(Y_{1} \cup_{F} Y_{2}\right) \cong H_{*}\left(\widehat{\mathrm{CFA}}\left(Y_{1}\right) \widetilde{\otimes} \widehat{\mathrm{CFD}}\left(Y_{2}\right)\right)=: \operatorname{Tor}_{\mathcal{A}(Z)}\left(\widehat{\mathrm{CFA}}\left(Y_{1}\right), \widehat{\mathrm{CFD}}\left(Y_{2}\right)\right) ;
$$

see [9], Theorem 1.3. (We review these constructions a little more thoroughly in Section 2.1.)

In this paper, we prove a different pairing theorem, formulated in terms of the Hom functor rather than the tensor product functor. This version has the advantage that it allows one to work exclusively with $\widehat{\mathrm{CFD}}(Y)$ (or, if one prefers, exclusively with $\widehat{\mathrm{CFA}}(Y)$ ); this is of interest since $\widehat{\mathrm{CFD}}$ is typically easier to compute (see [11]). The present pairing theorem also meshes well with the "Fukaya-categorical" formulation of Lagrangian Floer homology, providing a direct comparison of our pairing result with Auroux's construction of bordered Floer homology [1]. Indeed, our first result is the following, which also appears as Theorem 1.5 in [1]:

Theorem 1. Let $Y_{1}$ and $Y_{2}$ be bordered 3-manifolds with $\partial Y_{1}=\partial Y_{2}=F(\mathcal{Z})$. Then

$$
\begin{aligned}
& \widehat{\mathrm{HF}}\left(-Y_{1} \cup_{\partial} Y_{2}\right) \\
& \quad \cong H_{*}\left(\operatorname{Mor}_{\mathcal{A}(-Z)}\left(\widehat{\mathrm{CFD}}\left(Y_{1}\right), \widehat{\mathrm{CFD}}\left(Y_{2}\right)\right)\right)=: \operatorname{Ext}_{\mathcal{A}(-Z)}\left(\widehat{\mathrm{CFD}}\left(Y_{1}\right), \widehat{\mathrm{CFD}}\left(Y_{2}\right)\right) \\
& \quad \cong H_{*}\left(\operatorname{Mor}_{\mathcal{A}(\mathcal{Z})}\left(\widehat{\mathrm{CFA}}\left(Y_{1}\right), \widehat{\mathrm{CFA}}\left(Y_{2}\right)\right)\right)=: \operatorname{Ext}_{\mathcal{A}(\mathcal{Z})}\left(\widehat{\mathrm{CFA}}\left(Y_{1}\right), \widehat{\mathrm{CFA}}\left(Y_{2}\right)\right) .
\end{aligned}
$$

The Hom pairing theorem (Theorem 1) follows from the behavior of the bordered Floer invariants under orientation reversal, stated as a duality theorem relating $\widehat{\mathrm{CFD}}(Y)$ and $\widehat{\mathrm{CFA}}(-Y)$ (Theorem 2 below). Note that this is a different kind of duality from the relationship between $\widehat{\mathrm{CFD}}(Y)$ and $\widehat{\mathrm{CFA}}(Y)$ from [10], Corollary 1.1 and Theorem 16. In addition to studying orientation reversal, we also a prove duality theorems in two other contexts: one corresponding to conjugation of $\operatorname{spin}^{c}$ structure (Theorem 3) and another corresponding to reversing the Morse function on the surface (Theorem 13). We also prove analogues of Theorems 1 and 2 for bimodules (see Section 1.2).

As a tool for establishing Theorem 2, we use a Heegaard diagram discovered independently by Auroux [1] and Zarev [23] (see Section 4). Studying this diagram gives 
several algebraic results, including an algebraic Serre duality theorem (Theorem 10), and an interpretation of Hochschild cohomology as a knot Floer homology group (Corollary 11). It also leads to an in interpretation of the duality results from [10], Corollary 1.1, in terms of Koszul duality (Sections 5.4 and 8).

In spite of its aesthetic appeal, this Hom version of the pairing theorem is less economical than the original tensor product pairing theorem: the complex $\operatorname{Hom}\left(\widehat{\mathrm{CFD}}\left(Y_{1}\right)\right.$, $\left.\widehat{\mathrm{CFD}}\left(Y_{2}\right)\right)$ is typically much larger than $\widehat{\mathrm{CF}}\left(-Y_{1} \cup_{\partial} Y_{2}\right)$. (By contrast, for any Heegaard diagram respecting the decomposition $Y=-Y_{1} \cup_{\partial} Y_{2}$, the dimensions over $\mathbb{F}_{2}$ of the complex $\widehat{\mathrm{CF}}(Y)$ from [15] and the complex $\widehat{\mathrm{CFA}}\left(-Y_{1}\right) \otimes \widehat{\mathrm{CFD}}\left(Y_{2}\right)$ from [9] are the same.)

We now explain the duality theorems in more detail.

1.1. Dualities for bordered Floer modules. We start with the effect of orientationreversal on the bordered Floer invariants. To state it, it is convenient to work at the level of chain complexes, not homology. In particular, we let $\operatorname{Mor}_{A}(M, N)$ denote the chain complex of $\mathcal{A}_{\infty}$-morphisms from $M$ to $N$, whose homology is $\operatorname{Ext}_{A}(M, N)$ (cf. Section 2.2).

Theorem 2. Let $Y$ be a bordered 3-manifold, with boundary parameterized by $\varphi: F(\mathcal{Z}) \rightarrow \partial Y$. Let $-Y$ denote $Y$ with its orientation reversed and boundary parameterized by the same $\varphi$, viewed as a map $F(-Z) \rightarrow \partial(-Y)$. Then there are homotopy equivalences:

$$
\begin{aligned}
& \operatorname{Mor}_{\mathcal{A}(-Z)}\left({ }_{\mathcal{A}(-Z)} \widehat{\mathrm{CFD}}(Y), \mathcal{A}(-\mathcal{Z})\right) \simeq \widehat{\mathrm{CFA}}(-Y)_{\mathcal{A}(-Z)}, \\
& \operatorname{Mor}_{\mathcal{A}(Z)}\left(\widehat{\mathrm{CFA}}(Y)_{\mathcal{A}(Z)}, \mathcal{A}(Z)\right) \simeq{ }_{\mathcal{A}(Z)} \widehat{\mathrm{CFD}}(-Y) .
\end{aligned}
$$

The above result gives a direct relation between $\widehat{\mathrm{CFD}}$ and $\widehat{\mathrm{CFA}}$, with no orientation reversal, as follows. The algebra $\mathcal{A}(-\mathcal{Z})$ is the opposite algebra to $\mathcal{A}(\mathcal{Z})$. So, we can

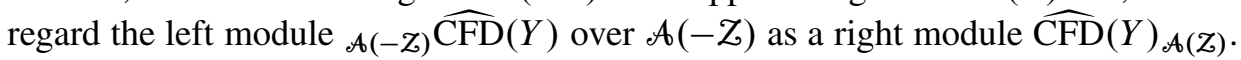
Recall also that the invariants $\widehat{\mathrm{CFD}}(Y)$ and $\widehat{\mathrm{CFA}}(Y)$ decompose according to (absolute) $\operatorname{spin}^{c}$-structures on $Y$,

$$
\widehat{\mathrm{CFD}}(Y)=\bigoplus_{\mathfrak{s} \in \operatorname{spin}^{c}(Y)} \widehat{\mathrm{CFD}}(Y, \mathfrak{s}), \quad \widehat{\mathrm{CFA}}(Y)=\bigoplus_{\mathfrak{s} \in \operatorname{spin}^{c}(Y)} \widehat{\mathrm{CFA}}(Y, \mathfrak{s}) .
$$

There is a $\mathbb{Z} / 2$-action on $\operatorname{spin}^{c}$-structures, called conjugation, and written $\mathfrak{s} \mapsto \overline{\mathfrak{s}}$.

Theorem 3. Let $Y$ be a bordered 3-manifold, with boundary parameterized by $F(\mathcal{Z})$, and let $\widehat{\mathrm{CFD}}(Y, \mathfrak{s})_{\mathcal{A}(\mathcal{Z})}$ denote the $\mathfrak{s}$-summand of ${ }_{\mathcal{A}(-Z)} \widehat{\mathrm{CFD}}(Y)$, viewed as a right module over $\mathcal{A}(Z)$. Then

$$
\widehat{\mathrm{CFD}}(Y, \mathfrak{s})_{\mathcal{A}(Z)} \cong \widehat{\mathrm{CFA}}(Y, \overline{\mathfrak{s}})_{\mathcal{A}(Z)} .
$$


(We are grateful to Denis Auroux for suggesting Theorem 3 to us.)

Heegaard Floer homology for closed three-manifolds satisfies a conjugation invariance property [16], Theorem 2.4. Theorem 3 gives the following version of conjugation invariance in the bordered theory.

Corollary 4. Let $Y$ be a bordered 3-manifold with boundary parameterized by $F(Z)$. Then

$$
\begin{gathered}
\widehat{\mathrm{CFA}}(Y, \overline{\mathfrak{s}})_{\mathcal{A}(\mathcal{Z})} \simeq\left(\widehat{\mathrm{CFA}}(Y, \mathfrak{s}) \bigotimes_{\mathcal{A}(\mathcal{Z})} \widehat{\mathrm{CFDD}}(\mathbb{I})\right)_{\mathcal{A}(Z)}, \\
\mathcal{A}(-\mathcal{Z}) \widehat{\mathrm{CFD}}(Y, \overline{\mathfrak{s}}) \simeq \mathcal{A}_{(-Z)}\left(\widehat{\mathrm{CFAA}}(\mathbb{I}) \bigotimes_{\mathcal{A}(-\mathcal{Z})} \widehat{\mathrm{CFD}}(Y, \mathfrak{s})\right)
\end{gathered}
$$

(Recall that, as in [9] and [10], we use $\otimes$ to denote a particularly convenient model for the $\mathcal{A}_{\infty}$ tensor product. Also, $\mathbb{I}$ denotes the identity map of $F(Z)$, and $\widehat{\mathrm{CFDD}}(\mathbb{I})$ and $\widehat{\mathrm{CFAA}}(\mathbb{I})$ the associated type $D D$ and $A A$ bimodules respectively. See Theorem 5.19 for an explicit description of $\widehat{\mathrm{CFDD}}(\mathbb{I})$.)

1.2. Analogues for bimodules. There are several analogues of these theorems for bimodules. When working with bimodules, many of the theorems require correcting by a boundary Dehn twist. Recall from [10] that to a bordered 3-manifold with two boundary components $F\left(\mathcal{Z}_{L}\right)$ and $F\left(\mathcal{Z}_{R}\right)$, together with a framed arc connecting the boundary components, one can associate a bimodule. We can, thus, talk about performing a boundary Dehn twist on such a strongly bordered 3-manifold, a Dehn twist along a loop surrounding the framed arc. (A boundary Dehn twist $\tau_{\partial}$ decreases the framing on the arc by 1.)

Before we can state the bimodule variants, we need one more algebraic digression. Suppose that $M$ is an $A-B$ bimodule which is free (or projective) as a bimodule, i.e. as a left $A \otimes B^{\text {op }}$ module. Then we can dualize $M$ over either one or both of the actions. That is, we can consider both of the bimodules $\operatorname{Hom}_{A}(M, A)$ and $\operatorname{Hom}_{A \otimes B}(M, A \otimes B)$. These are, in general, different bimodules. Analogous constructions exist in the $d g$ setting; see Section 2.3. These two algebraic operations lead to two different versions of the Hom pairing theorem for bimodules. The first of these is the following theorem.

Theorem 5. Suppose $Y$ is a strongly bordered 3-manifold with two boundary components $F\left(Z_{1}\right)$ and $F\left(Z_{2}\right)$. Then

$$
\begin{gathered}
\left.\operatorname{Mor}_{\mathcal{A}_{1}^{\prime}}\left(\mathcal{A}_{1}^{\prime}, \mathcal{A}_{2}^{\prime} \widehat{\operatorname{CFDD}}(Y), \mathcal{A}_{1}^{\prime}\right)\right) \simeq \widehat{\widehat{C F A A}}(-Y)_{\mathcal{A}_{1}^{\prime}, \mathcal{A}_{2}^{\prime},} \\
\operatorname{Mor}_{\mathcal{A}_{1}}\left(\widehat{\mathcal{A}_{2}^{\prime}} \widehat{\operatorname{CFDA}}(Y)_{\mathcal{A}_{1}}, \mathcal{A}_{1}\right) \simeq \mathscr{A}_{1} \widehat{\operatorname{CFDA}}(-Y)_{\mathcal{A}_{2}^{\prime}}, \\
\operatorname{Mor}_{\mathcal{A}_{1}^{\prime}}\left(\widehat{\mathcal{A}_{1}^{\prime}} \widehat{\operatorname{CFDA}}(Y)_{\mathcal{A}_{2}}, \mathcal{A}_{1}^{\prime}\right) \simeq \mathscr{A}_{2} \widehat{\operatorname{CFDA}}(-Y)_{\mathcal{A}_{1}^{\prime}}, \\
\operatorname{Mor}_{\mathscr{A}_{1}}\left(\widehat{\operatorname{CFAA}}(Y)_{\mathcal{A}_{1}, \mathcal{A}_{2}}, \mathcal{A}_{1}\right) \simeq \mathscr{A}_{1}, \mathcal{A}_{2} \widehat{\operatorname{CFDD}}(-Y) .
\end{gathered}
$$


(As explanation for the strange-looking notation, recall that, as defined in [10], the bimodule $\widehat{\operatorname{CFDD}}(Y)$ has two left actions, while the bimodule $\widehat{\mathrm{CFAA}}(Y)$ has two right actions. For convenience, we have written $\mathcal{A}_{i}$ for $\mathcal{A}\left(\mathcal{Z}_{i}\right)$ and $\mathcal{A}_{i}^{\prime}$ for $\mathcal{A}\left(-\mathcal{Z}_{i}\right)$.)

The second type of dualizing leads to the following theorem.

Theorem 6. Suppose $Y$ is a strongly bordered 3-manifold with two boundary components $F\left(Z_{1}\right)$ and $F\left(Z_{2}\right)$. Then

$$
\begin{aligned}
& \operatorname{Mor}_{\mathcal{A}_{1}^{\prime} \otimes \mathcal{A}_{2}^{\prime}}\left(\mathscr{A}_{1}^{\prime}, \mathcal{A}_{2}^{\prime} \widehat{\operatorname{CFDD}}(Y), \mathscr{A}_{1}^{\prime} \otimes_{\mathbb{F}_{2}} \mathcal{A}_{2}^{\prime}\right) \simeq \widehat{\operatorname{CFAA}}\left(-\tau_{\partial}^{-1}(Y)\right)_{\mathcal{A}_{1}^{\prime}, \mathcal{A}_{2}^{\prime}}, \\
& \left.\operatorname{Mor}_{\mathcal{A}_{1} \otimes \mathcal{A}_{2}}\left(\widehat{\mathrm{CFAA}}(Y)_{\mathcal{A}_{1}, \mathcal{A}_{2}}\right), \mathscr{A}_{1} \otimes_{\mathbb{F}_{2}} \mathcal{A}_{2}\right) \simeq{\mathcal{A}_{1}, \mathcal{A}_{2}}_{\widehat{C F D D}}\left(-\tau_{\partial}^{-1}(Y)\right) \text {. }
\end{aligned}
$$

(Here, in the notation we use the quasi-equivalence of categories between the category of right-right $\mathcal{A}_{\infty} \mathcal{A}_{1}-\mathcal{A}_{2}$-bimodules and the category of right $\mathcal{A}_{\infty} \mathcal{A}_{1} \otimes \mathcal{A}_{2}-$ modules. This equivalence is not as obvious as for ordinary bimodules; see, for instance, [10], Section 2.4.3, for further discussion. Alternately, one could replace Mor $_{\mathcal{A}_{1} \otimes \mathcal{A}_{2}}$ with the chain complex of $\mathcal{A}_{\infty}$-bimodule morphisms, as defined in [10], Section 2.2.4, say.)

One can obtain a version of Theorem 6 for $\widehat{\mathrm{CFDA}}$ by tensoring with $\widehat{\mathrm{CFDD}}(\mathbb{I})$ and using the fact that this gives an equivalence of categories (cf. the proof of (1.7) in Section 5); we leave this to the interested reader.

As in the one boundary component case, Theorems 5 and 6 lead to various pairing theorems. For example we have the following results.

Corollary 7. If $Y_{1}$ and $Y_{2}$ are strongly bordered 3-manifolds, with $\partial Y_{1}$ parameterized by $F\left(Z_{1}\right)$ and $F\left(Z_{2}\right)$ and $\partial Y_{2}$ parameterized by $F\left(Z_{1}\right)$ and $F\left(Z_{3}\right)$, then

$$
\begin{aligned}
& \mathcal{A}_{3}^{\prime} \widehat{\mathrm{CFDA}}\left(-Y_{1} \cup_{F\left(Z_{1}\right)} Y_{2}\right)_{\mathcal{A}_{2}^{\prime}} \\
& \simeq \operatorname{Mor}_{\mathcal{A}_{1}^{\prime}}\left(\mathscr{A}_{2}^{\prime}, \mathcal{A}_{1}^{\prime} \widehat{\operatorname{CFDD}}\left(Y_{1}\right), \mathscr{A}_{1}^{\prime}, \mathcal{A}_{3}^{\prime} \widehat{\operatorname{CFDD}}\left(Y_{2}\right)\right)
\end{aligned}
$$

and

$$
\begin{aligned}
& \mathcal{A}_{2}, \mathcal{A}_{3}^{\prime} \widehat{\mathrm{CFDD}}\left(\tau_{\partial}\left(-Y_{1} \cup_{\partial} Y_{2}\right)\right) \\
& \simeq \operatorname{Mor}_{\mathcal{A}_{1} \otimes \mathcal{A}_{1}^{\prime}}\left(\mathcal{A}_{1}, \mathcal{A}_{1}^{\prime} \widehat{\operatorname{CFDD}}(\mathbb{I}), \mathscr{A}_{1}, \mathcal{A}_{2} \widehat{\operatorname{CFDD}}\left(-Y_{1}\right) \otimes_{\mathcal{A}_{1}^{\prime}, \mathcal{A}_{3}^{\prime}} \widehat{\operatorname{CFDD}}\left(Y_{2}\right)\right) \text {. }
\end{aligned}
$$

(Here, as earlier, $\mathbb{I}$ denotes the identity map of $F(\mathcal{Z})$, and $\widehat{\mathrm{CFDD}}(\mathbb{I})$ the associated type $D D$ module.)

Corollary 8. If $Y_{1}$ is a strongly bordered 3-manifold with boundary parameterized by $F\left(Z_{1}\right)$ and $F\left(Z_{2}\right)$ and $Y_{2}$ is a bordered 3-manifold with boundary parameterized by $F\left(Z_{2}\right)$ then

$$
\begin{aligned}
& \widehat{\mathrm{CFA}}\left(-Y_{1} \cup_{F\left(\mathcal{Z}_{2}\right)} Y_{2}\right)_{\mathcal{A}_{1}^{\prime}} \simeq \operatorname{Mor}_{\mathcal{A}_{2}^{\prime}}\left(\mathcal{A}_{1}^{\prime}, \mathcal{A}_{2}^{\prime} \widehat{\operatorname{CFDD}}\left(Y_{1}\right),{ }_{\mathcal{A}_{2}^{\prime}} \widehat{\operatorname{CFD}}\left(Y_{2}\right)\right), \\
& \widehat{\mathcal{A}_{1}^{\prime}} \widehat{\mathrm{CFD}}\left(-Y_{2} \cup_{F\left(\mathcal{Z}_{2}\right)} Y_{1}\right) \simeq \operatorname{Mor}_{\mathcal{A}_{2}^{\prime}}\left(\mathcal{A}_{2}^{\prime} \widehat{\operatorname{CFD}}\left(Y_{2}\right),{ }_{\mathcal{A}_{1}^{\prime}, \mathcal{A}_{2}^{\prime}} \widehat{\operatorname{CFDD}}\left(Y_{1}\right)\right) .
\end{aligned}
$$


In particular, if $\psi: F\left(\mathcal{Z}_{2}\right) \rightarrow F\left(\mathcal{Z}_{1}\right)$ then

$$
\begin{aligned}
& \widehat{\mathrm{CFA}}\left(\psi\left(Y_{2}\right)\right)_{\mathcal{A}_{1}} \simeq \operatorname{Mor}_{\mathcal{A}_{2}^{\prime}}\left(\mathcal{A}_{1}, \mathcal{A}_{2}^{\prime} \widehat{\operatorname{CFDD}}\left(\psi^{-1}\right),{ }_{\mathcal{A}_{2}^{\prime}} \widehat{\operatorname{CFD}}\left(Y_{2}\right),\right. \\
& \mathcal{A}_{1}^{\prime} \widehat{\mathrm{CFD}}\left(\psi\left(Y_{2}\right)\right) \simeq \operatorname{Mor}_{\mathcal{A}_{2}}\left(\mathscr{A}_{2} \widehat{\operatorname{CFD}}\left(-Y_{2}\right),{ }_{\mathcal{A}_{1}^{\prime}, \mathcal{A}_{2}} \widehat{\operatorname{CFDD}}(\psi)\right) .
\end{aligned}
$$

There are also versions of Theorem 3 for bimodules.

Theorem 9. Suppose $Y$ is a strongly bordered 3-manifold with two boundary components $F\left(Z_{1}\right)$ and $F\left(Z_{2}\right)$. Then viewing ${ }_{\mathcal{A}_{1}^{\prime}, \mathcal{A}_{2}^{\prime}} \widehat{\operatorname{CFDD}}(Y)$ as a right-right module $\widehat{\operatorname{CFDD}}(Y)_{\mathcal{A}_{1}, \mathcal{A}_{2}}$ over $\mathcal{A}_{1}$ and $\mathcal{A}_{2}$,

$$
\widehat{\operatorname{CFDD}}(Y, \mathfrak{s})_{\mathcal{A}_{1}, \mathcal{A}_{2}} \simeq \widehat{\operatorname{CFAA}}\left(\tau_{\partial}(Y), \overline{\mathfrak{s}}\right)_{\mathcal{A}_{1}, \mathcal{A}_{2}} .
$$

Similarly, viewing $\widehat{\mathrm{CFAA}}(Y, \mathfrak{s})$ as a left-left module over $\mathcal{A}_{1}^{\prime}$ and $\mathcal{A}_{2}^{\prime}$, we have:

$$
\mathcal{A}_{1}^{\prime}, \mathcal{A}_{2}^{\prime} \widehat{\mathrm{CFAA}}(Y, \mathfrak{s}) \simeq \mathcal{A}_{1}^{\prime}, \mathcal{A}_{2}^{\prime} \widehat{\operatorname{CFDD}}\left(\tau_{\partial}^{-1}(Y), \overline{\mathfrak{s}}\right) \text {. }
$$

Finally, there are two versions of $\widehat{\mathrm{CFDA}}(Y)$, depending on whether we treat $F\left(Z_{1}\right)$ or $F\left(Z_{2}\right)$ as the type $D$ side. Denote these two modules by ${ }_{\mathcal{A}_{1}^{\prime}} \widehat{\operatorname{CFDA}}(Y, \mathfrak{s})_{\mathcal{A}_{2}}$ and $\mathscr{A}_{2}^{\prime} \widehat{\mathrm{CFDA}}(Y, \overline{\mathfrak{s}})_{\mathcal{A}_{1}}$, respectively. These two options are related by conjugation of the $\operatorname{spin}^{c}$-structure:

$$
\widehat{\mathcal{A}_{1}^{\prime}} \widehat{\mathrm{CFDA}}(Y, \mathfrak{s})_{\mathcal{A}_{2}} \simeq \widehat{\mathcal{A}_{2}^{\prime}} \widehat{\operatorname{CFDA}}(Y, \overline{\mathfrak{s}})_{\mathcal{A}_{1}} .
$$

Here, we mean that the modules are homotopy equivalent if we exchange the sidedness of the actions on either one of the two.

1.3. Algebraic consequences. Finally, these techniques can be used to prove several more algebraic results about the category of $\mathcal{A}(\mathbb{Z})$-modules.

Theorem 10. Given right $\mathcal{A}_{\infty}$-modules $M$ and $N$ over $\mathcal{A}(Z)$,

$$
\operatorname{Mor}_{\mathcal{A}(Z)}\left(N, M \otimes \widehat{\operatorname{CFDA}}\left(\tau_{\partial}^{-1}\right)\right) \simeq \operatorname{Mor}_{\mathcal{A}(\mathcal{Z})}(M, N)^{*},
$$

naturally. Here, * denotes the dual vector space.

In other words, Theorem 10 says that tensoring with $\widehat{\operatorname{CFDA}}\left(\tau_{\partial}^{-1}\right)$ is the Serre functor for the category of $\mathcal{A}(\mathcal{Z})$-modules.

In [10], we identified the Hochschild homology of $\widehat{\text { CFDA }}$ with a certain self-gluing operation. The algebraic results from this paper allow us to translate this result into one about Hochschild cohomology. (The non-specialist is reminded that Hochschild cohomology is typically not dual to Hochschild homology.) 
Corollary 11. Suppose that $Y$ is a strongly bordered 3-manifold with boundary $F(Z) \amalg F(-Z)$. Let $\tau_{\partial}(Y)$ denote the result of decreasing the framing on the arc $\mathbf{z}$ in $Y$ by one and $\tau_{\partial}(Y)^{\circ}$ the manifold obtained by gluing the two boundary components of $\tau_{\partial}(Y)$ together and performing surgery on the framed knot $K$ coming from the arc $\mathbf{z}$. Let $K^{\prime}$ be the knot in $\tau_{\partial}(Y)^{\circ}$ coming from $K$. Then the Hochschild cohomology $\mathrm{HH}^{*}(\widehat{\mathrm{CFDA}}(Y))$ is isomorphic to $\widehat{\mathrm{HFK}}\left(\tau_{\partial}(Y)^{\circ}, K^{\prime}\right)$.

The following theorem was first proved in [10], Theorem 4, using computations of the homology of the algebra associated to a pointed matched circle. The techniques of this paper lead to another proof, which is independent of those calculations.

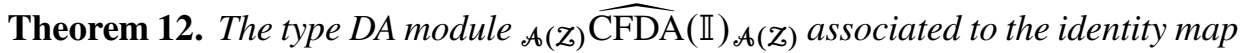

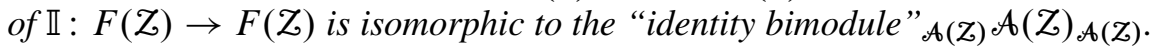

The algebras $\mathcal{A}(\mathcal{Z})$ have yet more symmetries. To state them, we give one more operation on pointed matched circles. Given a pointed matched circle $\mathcal{Z}$, we can form another pointed matched circle $\mathcal{Z}_{*}$ by turning the Morse function inducing $\mathcal{Z}$ upside down; see Construction 8.18 for more details.

Theorem 13. The algebra $\mathcal{A}(\mathcal{Z}, i)$ is Koszul dual to $\mathcal{A}(\mathcal{Z},-i)$ and also to $\mathcal{A}\left(\mathcal{Z}_{*}, i\right)$. In particular, $\mathcal{A}\left(\mathcal{Z}_{,},-i\right)$ is quasi-isomorphic to $\mathcal{A}\left(\mathcal{Z}_{*}, i\right)$.

(Our algebras have differentials and are not strictly quadratic, so the definition of Koszul duality in our setting is a modest extension of the classical one. See Section 8.1.)

Theorem 13 explains some seeming coincidences in $\operatorname{dim}\left(H_{*}(\mathcal{A}(\mathcal{Z}, i))\right)$; see Section 8.2 for some examples.

As mentioned earlier, a key tool for establishing these results is a Heegaard diagram discovered independently by Auroux [1] and Zarev (see Section 4). This is a nice diagram [20], so its holomorphic disks can be understood explicitly; moreover, its combinatorial structure is closely tied to the bordered Floer algebra. This allows us to describe differentials in this diagram in a reasonably conceptual way. Exploiting these properties, we can explicitly describe some modules which play an important role in the theory. For instance, in Theorem 5.19 we give a simple description of the dualizing bimodule $\widehat{\mathrm{CFDD}}(\mathbb{I})$ (which is also computed, by different techniques, in [11]). In a similar vein, we can give a conceptual description of the Serre functor appearing above and a finite dimensional model for the bar complex of $\mathcal{A}$ (see Proposition 5.13 below).

1.4. Gradings. We have stated the results in the introduction without explicitly discussing the gradings on the modules and bimodules. Typically, gradings are somewhat subtle in bordered Floer theory; in particular, the algebras are graded by noncommutative groups and the modules by $G$-sets. It turns out, however, that these issues 
do not introduce any novel features for the results in this paper, beyond those already present in the pairing theorems from [9] and [10]. We review these issues, in Section 6. There, we also give a detailed statement of how the gradings work in Theorem 1; graded statements of the other theorems are similar, and we leave these to the reader.

1.5. Further remarks. It is natural to ask what operation in Heegaard Floer homology corresponds to the composition of homomorphisms. That is, suppose we have bordered 3-manifolds $Y_{1}, Y_{2}$ and $Y_{3}$ with boundaries parametrized by some surface $F$. Then there is a composition map

$$
\operatorname{Ext}\left(\widehat{\mathrm{CFD}}\left(Y_{1}\right), \widehat{\mathrm{CFD}}\left(Y_{2}\right)\right) \otimes \operatorname{Ext}\left(\widehat{\mathrm{CFD}}\left(Y_{2}\right), \widehat{\mathrm{CFD}}\left(Y_{3}\right)\right) \longrightarrow \operatorname{Ext}\left(\widehat{\mathrm{CFD}}\left(Y_{1}\right), \widehat{\mathrm{CFD}}\left(Y_{3}\right)\right.
$$

which corresponds to some homomorphism

$$
\widehat{\mathrm{HF}}\left(-Y_{1} \cup_{\partial} Y_{2}\right) \otimes \widehat{\mathrm{HF}}\left(-Y_{2} \cup_{\partial} Y_{3}\right) \longrightarrow \widehat{\mathrm{HF}}\left(-Y_{1} \cup_{\partial} Y_{3}\right) .
$$

Generalizing a notion from [15], we can use $Y_{1}, Y_{2}$ and $Y_{3}$ to construct a 4-manifold as follows. Let $T$ denote a triangle, with edges $e_{1}, e_{2}$ and $e_{3}$. Then let

$$
W_{Y_{1}, Y_{2}, Y_{3}}=(T \times F) \cup_{e_{1} \times F}\left(e_{1} \times Y_{1}\right) \cup_{e_{2} \times F}\left(e_{2} \times Y_{2}\right) \cup_{e_{3} \times F}\left(e_{3} \times Y_{3}\right) .
$$

Following constructions from [17], this four-manifold induces a map on Floer homology

$$
\hat{F}_{W_{Y_{1}, Y_{2}, Y_{3}}}: \widehat{\mathrm{HF}}\left(-Y_{1} \cup_{\partial} Y_{2}\right) \otimes \widehat{\mathrm{HF}}\left(-Y_{2} \cup_{\partial} Y_{3}\right) \longrightarrow \widehat{\mathrm{HF}}\left(-Y_{1} \cup_{\partial} Y_{3}\right) .
$$

Under the identifications from Theorem 1, one can show that this four-manifold invariant corresponds to the composition map

$$
\operatorname{Ext}\left(\widehat{\mathrm{CFD}}\left(Y_{1}\right), \widehat{\mathrm{CFD}}\left(Y_{2}\right)\right) \otimes \operatorname{Ext}\left(\widehat{\mathrm{CFD}}\left(Y_{2}\right), \widehat{\mathrm{CFD}}\left(Y_{3}\right)\right) \longrightarrow \operatorname{Ext}\left(\widehat{\mathrm{CFD}}\left(Y_{1}\right), \widehat{\mathrm{CFD}}\left(Y_{3}\right)\right)
$$

Presumably a similar story holds for the multiplication operations on Hochschild cohomology. We return to this point in a future paper [7].

The duality results for modules and bimodules can be seen as special cases of results for bordered sutured manifolds [22]. In that, more general, context the presence of boundary Dehn twists can be understood as follows. If $Y$ is a sutured manifold then orientation reversal of $Y$ also reverses the roles of $R_{+}$and $R_{-}$. To accommodate this difference, one must also introduce a "half Dehn twist" along the bordered part of the boundary of $Y_{1}$ (compare Definition 3.6) before gluing it to $Y_{2}$. Though we will generally not discuss this case, see Remark 5.1 for a little more on subtleties in the bordered sutured context.

In a different direction, one must proceed with care in adapting Theorem 1 to the case of bordered sutured manifolds [22]. This extension is developed (in a slightly different language) in [23]; see also Remark 5.1. 
1.6. Outline of the paper. In Section 2 we review some basic facts about bordered Floer theory and homological algebra which are used in the rest of this paper. A key point in the present paper is to generalize the arced bordered Heegaard diagrams from [10] to the case where both $\alpha$ and $\beta$ curves go out to the boundary, and to give a topological meaning to these objects. This is done in Section 3. In Section 4 we describe a particular Heegaard diagram whose associated bimodule is the algebra itself. This lets us give Heegaard-diagrammatic interpretations to some of the algebraic operations on the various modules in bordered Floer theory. In Section 5, we collect the consequences of these interpretations (together with the traditional pairing theorem) to prove the results stated in the introduction. In Section 6 we give a brief discussion of how gradings can be added to the present context. In Section 7 we illustrate some of the above discussion with some examples. Finally, in Section 8, we give a description of Koszul duality relevant for our algebras, and prove Theorem 13.

A summary of some of the conventions employed in the paper can be found in Appendix A.

Acknowledgements. The authors thank Denis Auroux and Rumen Zarev for helpful conversations. In particular, Auroux's paper [1] led us to a dramatically simplified argument. The relevant diagram of Auroux was discovered (and communicated to us) independently by Zarev. As noted above, Theorem 1 was discovered independently by Auroux, and a form of Theorem 3 was also suggested to us by him. The authors also thank MSRI for its hospitality during the completion of this project. Finally, we thank the referees for a detailed reading and many helpful and interesting comments.

\section{Background}

2.1. Basic structure of bordered Floer theory. We start reviewing the key ingredients from [9] and [10].

A matched circle is an oriented circle $Z, 2 k$ points $\left\{a_{1}, \ldots, a_{2 k}\right\}=\mathbf{a} \subset Z$ and a fixed-point free involution $M: \mathbf{a} \rightarrow \mathbf{a}$. A matched circle $(Z, \mathbf{a}, M)$ specifies a surface-with-boundary $F^{\circ}(Z, \mathbf{a}, M)$ by filling in $Z$ with a disk $D_{0}$ and attaching 2-dimensional 1-handles at each pair $\left\{a_{i}, a_{M(i)}\right\} \subset$ a. We shall only be interested in matched circles which specify surfaces with a single boundary component. We can fill in the boundary component of $F^{\circ}(Z, \mathbf{a}, M)$ to give a closed surface $F$, with a distinguished disk $D=F \backslash F^{\circ}$. We orient $F$ so that the orientation of $D_{0}$ induces the orientation of $Z$.

A pointed matched circle is a matched circle together with a basepoint $z \in Z \backslash \mathbf{a}$. We shall use the notation $Z$ to denote a pointed matched circle $(Z, \mathbf{a}, M, z)$. A pointed matched circle $Z$ specifies a closed surface $F(Z)$ together with a distinguished disk $D \subset F(\mathcal{Z})$ and basepoint $z \in \partial D$. (This construction will be expanded slightly in Section 3.1.) 
Bordered Floer homology associates a $d g$ algebra $\mathcal{A}(\mathcal{Z})$ to each pointed matched circle [9]. If $F(\mathbb{Z}) \cong F\left(\mathbb{Z}^{\prime}\right)$ then the algebras $\mathcal{A}(\mathbb{Z})$ and $\mathcal{A}\left(\mathbb{Z}^{\prime}\right)$ are derived equivalent, according to [10], Theorem 1.

A bordered 3-manifold is a quadruple $\left(Y_{1}, \Delta_{1}, z_{1}, \psi_{1}\right)$, where $Y_{1}$ is a threemanifold-with-boundary, $\Delta_{1}$ is a disk in $\partial Y_{1}, z_{1}$ is a point on $\partial \Delta_{1}$, and

$$
\psi:(F(Z), D, z) \longrightarrow\left(\partial Y_{1}, \Delta_{1}, z_{1}\right),
$$

is a homeomorphism from the surface $F(\mathbb{Z})$ (for some pointed matched circle $Z$ ) to $\partial Y_{1}$ sending $D$ to $\Delta_{1}$ and $z$ to $z_{1}$. We will often suppress the preferred disk $D$ and basepoint $z$ from the notation for a bordered 3-manifold, and will sometimes also suppress the homeomorphism $\psi$.

As explained in [9], bordered Floer homology associates to a bordered 3-manifold with boundary parameterized by $F(Z)$ a right $\mathcal{A}_{\infty} \mathcal{A}(Z)$-module $\widehat{\mathrm{CFA}}(Y)$ and a left $d g \mathcal{A}(-Z)$-module $\widehat{\mathrm{CFD}}(Y)$, each well-defined up to quasi-isomorphism. (Here, $-\mathbb{Z}$ denotes $Z$ with its orientation reversed.)

Bordered Floer homology also works for 3-manifolds with more than one boundary component; see [10]. More precisely, a strongly bordered three-manifold with boundary $F\left(Z_{1}\right) \amalg F\left(Z_{2}\right)$ is an oriented three manifold $Y_{12}$ with boundary, equipped with

- preferred disks $\Delta_{1}$ and $\Delta_{2}$ on its two boundary components,

- basepoints $z_{i}^{\prime} \in \partial \Delta_{i}$,

- a homeomorphism $\psi:\left(F\left(Z_{1}\right) \amalg F\left(Z_{2}\right), D_{1} \amalg D_{2}, z_{1} \amalg z_{2}\right) \rightarrow\left(\partial Y_{12}, \Delta_{1} \amalg \Delta_{2}\right.$, $\left.z_{1}^{\prime} \amalg z_{2}^{\prime}\right)$,

- an arc $\gamma$ connecting $z_{1}^{\prime}$ to $z_{2}^{\prime}$, and

- a framing of $\gamma$, pointing into $\Delta_{i}$ at $z_{i}^{\prime}$ for $i=1,2$.

We will often denote the two boundary components of a strongly bordered threemanifold $Y$ by $\partial_{L} Y$ and $\partial_{R} Y$ (for "left" and "right"), but the choice of which boundary component is $\partial_{L}$ and which is $\partial_{R}$ is arbitrary.

For every strongly bordered 3-manifold with boundary $F\left(\mathcal{Z}_{1}\right) \amalg F\left(Z_{2}\right)$, bordered Floer homology associates an $\mathcal{A}_{\infty}$-bimodule $\widehat{\mathrm{CFAA}}(Y)$ with right actions by $\mathcal{A}\left(\mathcal{Z}_{1}\right)$ and $\mathcal{A}\left(Z_{2}\right)$; an $\mathcal{A}_{\infty}$-bimodule $\widehat{\mathrm{CFDA}}(Y)$ with a left action by $\mathcal{A}\left(-Z_{1}\right)$ and a right action by $\mathcal{A}\left(\mathcal{Z}_{2}\right)$; and a $d g$ bimodule $\widehat{\operatorname{CFDD}}(Y)$ with left actions by $\mathcal{A}\left(-Z_{1}\right)$ and $\mathcal{A}\left(-Z_{2}\right)$. Each of $\widehat{\mathrm{CFAA}}(Y), \widehat{\mathrm{CFDA}}(Y)$ and $\widehat{\mathrm{CFDD}}(Y)$ is well-defined up to quasiisomorphism. As a special case, if $\mathbb{Z}$ is a pointed matched circle, $F(\mathbb{Z}) \times[0,1]$ is naturally a strongly bordered three-manifold, which we will denote $\mathbb{I}_{\mathcal{Z}}$ or just $\mathbb{I}$.

The bordered Floer modules relate to each other and to the closed invariant $\widehat{\mathrm{CF}}(Y)$ by pairing theorems. The prototypical pairing theorem (see [9], Theorem 1.3) states that if $Y_{1}$ and $Y_{2}$ are bordered 3-manifolds with $\partial Y_{1}=F(\mathcal{Z})=-\partial Y_{2}$ then

$$
\widehat{\mathrm{CF}}\left(Y_{1} \cup_{F} Y_{2}\right) \simeq \widehat{\mathrm{CFA}}\left(Y_{1}\right) \widetilde{\otimes}_{\mathcal{A}(Z)} \widehat{\mathrm{CFD}}\left(Y_{2}\right)
$$


Here, $\widetilde{\otimes}$ denotes the derived (or $\mathcal{A}_{\infty}$ ) tensor product. The analogues for bimodules are listed in [10]; the mnemonic is that one can cancel expressions of the form $A \widetilde{\otimes} D$. For instance, if $Y_{1}$ is a bordered 3-manifold with boundary $F\left(Z_{1}\right)$ and $Y_{12}$ and $Y_{23}$ are strongly bordered 3-manifolds with boundaries $-F\left(\mathcal{Z}_{1}\right) \amalg F\left(\mathcal{Z}_{2}\right)$ and $-F\left(Z_{2}\right) \amalg F\left(Z_{3}\right)$ respectively then

$$
\begin{aligned}
\widehat{\operatorname{CFD}}\left(Y_{1} \cup_{F\left(Z_{1}\right)} Y_{12}\right) & \simeq \widehat{\operatorname{CFA}}\left(Y_{1}\right) \tilde{\otimes}_{\mathcal{A}\left(Z_{1}\right)} \widehat{\operatorname{CFDD}}\left(Y_{12}\right), \\
\widehat{\operatorname{CFDA}}\left(Y_{12} \cup_{F\left(Z_{2}\right)} Y_{23}\right) & \simeq \widehat{\operatorname{CFA}}\left(Y_{12}\right) \widetilde{\otimes}_{\mathcal{A}\left(Z_{2}\right)} \widehat{\operatorname{CFDD}}\left(Y_{23}\right),
\end{aligned}
$$

and so on.

The details of the construction of the algebras $\mathcal{A}(F)$ and the modules $\widehat{\mathrm{CFD}}(Y)$ and $\widehat{\mathrm{CFA}}(Y)$ can be found in [9], and the generalization to the case of more boundary components is in [10]. Much of this paper can be read with merely a cursory understanding of [9] and [10], keeping the following points in mind.

- Suppose that $\mathbb{Z}$ is a pointed matched circle and $-\mathbb{Z}$ denotes the same data except with the orientation of the circle reversed. Then $F(-\mathbb{Z})=-F(\mathbb{Z})$, and the algebras are related by:

$$
\mathcal{A}(Z)^{\mathrm{op}} \cong \mathcal{A}(-Z) .
$$

- The modules $\widehat{\mathrm{CFD}}(Y)$ and $\widehat{\mathrm{CFA}}(Y)$ are not associated directly to the 3-manifold $Y$, but rather to a bordered Heegaard diagram for $Y$, i.e. a Heegaard diagram

$$
\left(\Sigma_{g}^{\prime}, \alpha^{c}=\left\{\alpha_{1}^{c}, \ldots, \alpha_{g-k}^{c}\right\}, \beta^{c}=\left\{\beta_{1}, \ldots, \beta_{g}\right\}, z\right)
$$

for $Y$ together with $2 k$ disjoint, embedded $\operatorname{arcs} \alpha^{a}=\left\{\alpha_{1}^{a}, \ldots, \alpha_{2 k}^{a}\right\}$ in $\bar{\Sigma}=$ $\Sigma^{\prime} \backslash \mathbb{D}^{2}$ with boundary on $\partial \bar{\Sigma}$ giving a basis for $\pi_{1}(\partial Y)$; and a basepoint $z \in \partial \bar{\Sigma}$ not lying on any $\alpha_{i}^{a}$. The boundary of such a diagram is a pointed matched circle.

Similarly, the bimodules $\widehat{\mathrm{CFDD}}(Y), \widehat{\mathrm{CFDA}}(Y)$ and $\widehat{\mathrm{CFAA}}(Y)$ associated to a strongly bordered 3-manifold with two boundary components are associated to arced bordered Heegaard diagrams with two boundary components; see [10] and also Section 3.3 below.

- The module $\widehat{\operatorname{CFD}}(Y)$ has a special form: it is a type $D$ structure, as defined below.

Definition 2.2. Let $\mathcal{A}$ be a $d g$ algebra over a ring $\mathbb{k}=\bigoplus_{i=1}^{N} \mathbb{F}_{2}$. A (left) type $D$ structure over $\mathcal{A}$ is a $\mathbb{k}$-module $X$ equipped with a map $\delta^{1}: X \rightarrow \mathcal{A} \otimes_{\mathbb{k}} X$ satisfying the structure equations which ensure that $\delta^{1}$ extends via the Leibniz rule to give $\mathcal{A} \otimes_{\mathbb{k}} X$ the structure of a differential $\mathcal{A}$-module.

(Type $D$ structures can be thought of as differential comodules or twisted complexes; see [10], Remark 2.2.36, and [10], Remark 2.2.37. respectively.) 
There is a convenient model $\square$ for the $\mathcal{A}_{\infty}$-tensor product of an $\mathscr{A}_{\infty}$-module $L$ and a type $D$ structure $M=\left(X, \delta^{1}\right)$, with the property that the vector space underlying $L \otimes M$ is just $L \otimes_{\mathbb{k}} X$; see [9], Definition 2.26. (In the case that $L$ is an ordinary module, $L \otimes M$ agrees with the naïve tensor product $L \otimes_{\mathcal{A}} M$. In particular, when $L$ is $\mathcal{A}$ viewed as a bimodule, $\mathcal{A} \otimes M$ is the module associated to $X$.) There are analogues of the operation $\otimes$ for bimodules, as well; see [10], Section 2.3.2. (Although $\otimes$ has a purely algebraic definition, it arises naturally in the analysis of pseudoholomorphic curves, as seen in the proof of the pairing theorem; see [9], Chapter 9.)

Similarly, the bimodule $\widehat{\operatorname{CFDD}}(Y)$ is a type $D D$ structure, i.e. a type $D$ structure over $\mathcal{A}\left(Z_{L}\right) \otimes \mathcal{A}\left(Z_{R}\right)$; and the bimodule $\widehat{\mathrm{CFDA}}(Y)$ is a type $D A$ structure, as defined in [10], Definition 2.2.43. The operation $\triangle$ works when tensoring bimodules, as long as one tensors a type $D$ side with a type $A$ (i.e. $\left.\mathcal{A}_{\infty}\right)$ side.

We sometimes blur the distinction between a type $D$ structure $\left(X, \delta^{1}\right)$ and its induced differential module $\mathcal{A} \otimes X$. When it is important to distinguish them, we include a superscript in the notation for a type $D$ structure, ${ }^{\mathcal{A}} X$. Ordinary modules are indicated with a subscript; so we sometimes use the notation ${ }_{\mathcal{A}} X$ to denote the associated module $\mathcal{A} \otimes{ }^{\mathcal{A}} X$. This operation has an inverse ("raising the subscript") which associates to a module $M_{\mathcal{A}}$ the type $D$ structure $M_{\mathcal{A}} \otimes{ }^{\mathcal{A}} \operatorname{Bar}(\mathcal{A})^{\mathcal{A}}$, the $b a r$ resolution of $M$. These are inverses in the derived category of modules satisfying suitable boundedness conditions; see [10], Proposition 1.3.18.

2.2. Review of Mor and Ext. Suppose that $C_{*}$ and $D_{*}$ are chain complexes (or differential modules) over an algebra $A$ (possibly with differential) which we assume to have characteristic 2. Two ways to compute $\operatorname{Ext}_{R}\left(C_{*}, D_{*}\right)$ are:

(1) find a complex $C_{*}^{\prime}$ of projective modules quasi-isomorphic to $C_{*}$ and compute the homology of the complex $\operatorname{Hom}_{A}\left(C_{*}^{\prime}, D_{*}\right)_{k}=\bigoplus_{i} \operatorname{Hom}\left(C_{i}^{\prime}, D_{k+i}\right)$ of maps respecting the module structure (but not necessarily the differential) from $C_{*}^{\prime}$ to $D_{*}$, or

(2) take the homology of the chain complex $\operatorname{Mor}_{A}\left(C_{*}, D_{*}\right)$ of $\mathcal{A}_{\infty}$-morphisms from $C_{*}$ to $D_{*}$.

(The second option is, under some finite-dimensionality assumptions, a special case of the first: the complex of $\mathcal{A}_{\infty}$-morphisms from $C_{*}$ to $D_{*}$ is exactly the chain complex of homomorphisms from the bar resolution of $C_{*}$ to $D_{*}$; see Section 2.3.)

Of course, if $C_{*}$ is already projective, one can take $C_{*}^{\prime}$ to just be $C_{*}$ itself. Given type $D$ structures ${ }^{\mathcal{A}} M$ and ${ }^{\mathcal{A}} N$, define

$$
\operatorname{Mor}^{\mathcal{A}}\left({ }^{\mathcal{A}} M,{ }^{\mathcal{A}} N\right)_{k}=\bigoplus_{i} \operatorname{Hom}\left({ }_{\mathcal{A}} M_{i}, A_{\mathcal{A}} N_{k+i}\right),
$$


with its obvious differential. Type $D$ structures over our algebras correspond to projective modules (compare [10], Corollary 2.3.25), so

$$
\operatorname{Ext}_{*}\left({ }_{\mathscr{A}} M,{ }_{\mathcal{A}} N\right) \cong H_{*}\left(\operatorname{Mor}^{\mathcal{A}}\left({ }^{\mathcal{A}} M,{ }^{\mathcal{A}} N\right)\right) \text {. }
$$

Again, the notation Mor $_{\mathcal{A}}$, with a subscript, denotes the complex of $\mathcal{A}_{\infty}$-morphisms between $\mathcal{A}_{\infty}$-modules, while the notation $\operatorname{Mor}^{\mathcal{A}}$, with a superscript, denotes the complex of module maps of type $D$ structures. In either case, the homology of the Mor complex computes Ext.

The following result of [10] (an easy consequence of [10], Theorems 4 and 12, see [10], Lemma 9.1) will reduce our work by roughly half.

Theorem 2.3. Fix a pointed matched circle Z. Then the functors

$$
\begin{aligned}
& \cdot \otimes \widehat{\mathrm{CFDD}}(\mathbb{I}): H_{*}\left(\operatorname{Mod}_{\mathcal{A}(\mathcal{Z})}\right) \longrightarrow H_{*}\left({ }^{\mathcal{A}(-\mathcal{Z})} \operatorname{Mod}\right) \\
& \widehat{\mathrm{CFAA}}(\mathbb{I}) \otimes \cdot: H_{*}\left({ }^{\mathcal{A}(-\mathcal{Z})} \operatorname{Mod}\right) \longrightarrow H_{*}\left(\operatorname{Mod}_{\mathcal{A}(\mathcal{Z})}\right)
\end{aligned}
$$

are inverse equivalences of categories, exchanging $\widehat{\mathrm{CFA}}(Y)$ and $\widehat{\mathrm{CFD}}(Y)$.

Here, $H_{*}\left(\operatorname{Mod}_{\mathscr{A}(\mathcal{Z})}\right)\left(\right.$ respectively $\left.H_{*}\left({ }^{\mathcal{A}(-\mathcal{Z})} \operatorname{Mod}\right)\right)$ denotes the homotopy category of right, $\mathcal{A}_{\infty}$ (respectively left, type $D$ ) modules over $\mathcal{A}(Z)$ (respectively $\mathcal{A}(-Z)$ ). Recall also that the homotopy categories of $\mathcal{A}_{\infty}$-modules and projective modules are both equivalent to the derived category; see [10], Section 2.4.1.

Corollary 2.4. Fix bordered 3-manifolds $Y_{1}$ and $Y_{2}$ with $\partial Y_{1}=F=\partial Y_{2}$. Then there is a quasi-isomorphism

$$
\operatorname{Mor}_{\mathcal{A}(F)}\left(\widehat{\mathrm{CFA}}\left(Y_{1}\right), \widehat{\mathrm{CFA}}\left(Y_{2}\right)\right) \simeq \operatorname{Mor}^{\mathcal{A}(-F)}\left(\widehat{\mathrm{CFD}}\left(Y_{1}\right), \widehat{\mathrm{CFD}}\left(Y_{2}\right)\right) .
$$

Although we do not need it for our present purposes, the module $\widehat{\operatorname{CFDD}}(\mathbb{I})$ is described explicitly in Section 5.4 or, via a different method, in [11].

2.3. Duals of modules and type $\boldsymbol{D}$ structures. As for finite-dimensional vector spaces, where $\operatorname{Hom}(V, W) \cong W \otimes V^{*}$, we can interpret our Mor complexes in terms of tensor products and duals. We spell this out explicitly.

Definition 2.5. Let $\mathcal{A}$ be a finite-dimensional $d g$ algebra over $\mathbb{k}=\bigoplus_{i=1}^{N} \mathbb{F}_{2}$ and $M=\left(X, \delta^{1}\right)$ a left type $D$ structure over $\mathcal{A}$. Let $\bar{X}=\operatorname{Hom}_{\mathbb{F}_{2}}\left(X, \mathbb{F}_{2}\right)$ denote the dual of $X$. The transpose of $\delta^{1}$ is a map

$$
\left(\delta^{1}\right)^{T}: \bar{X} \otimes \operatorname{Hom}_{\mathbb{F}_{2}}\left(\mathcal{A}, \mathbb{F}_{2}\right) \longrightarrow \bar{X} .
$$

We can interpret this instead as a map

$$
\bar{\delta}^{1}: \bar{X} \longrightarrow \bar{X} \otimes \mathcal{A} .
$$

The dual type $D$ structure to $M, \bar{M}$, is the right type $D$ structure induced by $\left(\bar{X}, \bar{\delta}^{1}\right)$. 
If we draw the operation on a type $D$ structure $M$ like this:

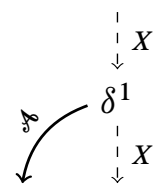

(compare [10], Section 2) then the type $D$ structure $\bar{M}$ is

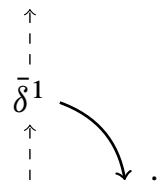

(As is standard in such graphical calculus, arrows pointing up represent the dual modules of arrows pointing down.)

Lemma 2.6. If $M=\left(X, \delta^{1}\right)$ is a type $D$ structure then $\bar{M}=\left(\bar{X}, \bar{\delta}^{1}\right)$ is also a type D structure.

Proof. This is a straightforward exercise in the properties of duals; alternately, it is clear from the graphical description.

Proposition 2.7. Suppose that $M$ and $N$ are type $D$ structures and at least one of $M$ or $N$ is finite-dimensional. Then the chain complex of module homomorphisms from $M$ to $N, \operatorname{Mor}^{\mathcal{A}}(M, N)$, is isomorphic to $\bar{M} \otimes_{\mathcal{A}} \mathcal{A}_{\mathbb{A}_{\mathcal{A}}} N$. In particular, $\operatorname{Mor}_{\mathcal{A}}\left({ }_{\mathcal{A}} M, \mathcal{A}\right) \simeq \bar{M} \otimes_{\mathcal{A}} \mathcal{A}$.

Proof. The first part is straightforward from the definitions. For the last statement, consider the type $D$ structure ${ }^{\mathcal{A}} T=(\mathbb{k}, 0)$. (That is, $T$ is rank one and $\left.\delta_{T}^{1}=0\right)$. Then ${ }_{\mathcal{A}} T=\mathcal{A}$ and $\bar{M} \otimes_{\mathcal{A}} \mathcal{A} \cong \operatorname{Mor}^{\mathcal{A}}\left({ }^{\mathcal{A}} M, T\right) \simeq \operatorname{Mor}_{\mathcal{A}}\left(\mathcal{A}_{\mathcal{A}} M, \mathcal{A}\right)$.

We next turn to duals of $\mathcal{A}_{\infty}$-modules.

Definition 2.8. Let $\mathcal{A}$ be a finite-dimensional $d g$ algebra over $\mathbb{k}=\bigoplus_{i=1}^{N} \mathbb{F}_{2}$ and $M$ a right $\mathcal{A}_{\infty}$-module over $\mathcal{A}$. Let $\bar{M}=\operatorname{Hom}_{\mathbb{F}_{2}}\left(M, \mathbb{F}_{2}\right)$. The higher multiplications $m_{i+1}: M \otimes \mathcal{A}^{\otimes i} \rightarrow M$ dualize to give maps $m_{i+1}^{T}: \bar{M} \rightarrow \operatorname{Hom}\left(\mathcal{A}, \mathbb{F}_{2}\right)^{\otimes i} \otimes \bar{M}$, which we can interpret as maps

$$
\bar{m}_{i+1}: \mathcal{A}^{\otimes i} \otimes \bar{M} \longrightarrow \bar{M}
$$

Then the data $\left(\bar{M},\left\{\bar{m}_{i+1}\right\}\right)$ is the dual $\mathcal{A}_{\infty}$-module to $M$. 
Lemma 2.9. If $\left(M,\left\{m_{i+1}\right\}\right)$ is a right $\mathcal{A}_{\infty}$-module then $\left(\bar{M},\left\{\bar{m}_{i+1}\right\}\right)$ also satisfies the $\mathcal{A}_{\infty}$-module relation to make $\bar{M}$ a left $\mathcal{A}_{\infty}$-module.

Proof. Again, this is a straightforward exercise in the properties of duals.

Morphism spaces of type $A$ modules can also be described in terms of $\otimes$. Before giving the definition, we recall some notation. An augmentation of a $d g$ algebra $\mathcal{A}$ is a homomorphism $\varepsilon: \mathcal{A} \rightarrow \mathbb{k}$ from the algebra to the ground ring. Given an augmentation $\varepsilon$ of a $d g$ algebra $\mathcal{A}=(A, \mu, d)$, let $A_{+}=\operatorname{ker}(\varepsilon)$ denote the augmentation ideal. There is a type $D D$ bimodule ${ }^{\mathcal{A}} \operatorname{Bar}_{r}(\mathcal{A})^{\mathcal{A}}$ with underlying $\mathbb{k}$ module $\mathcal{T}^{*}\left(A_{+}\right)$, with basis written $\left[a_{1}|\cdots| a_{k}\right]$ for $k \geq 0$, and structure maps

$$
\begin{aligned}
\delta^{1}\left[a_{1}|\cdots| a_{k}\right]:= & a_{1} \otimes\left[a_{2}|\cdots| a_{k}\right] \otimes 1+1 \otimes\left[a_{1}|\cdots| a_{k-1}\right] \otimes a_{k} \\
& +\sum_{1 \leq i \leq k} 1 \otimes\left[a_{1}|\cdots| d\left(a_{i}\right)|\cdots| a_{k}\right] \otimes 1 \\
& +\sum_{1 \leq i \leq k-1} 1 \otimes\left[a_{1}|\cdots| \mu\left(a_{i}, a_{i+1}\right)|\cdots| a_{k}\right] \otimes 1 .
\end{aligned}
$$

The bimodule ${ }^{\mathcal{A}} \mathrm{Bar}_{r}(\mathcal{A})^{\mathscr{A}}$ is called the reduced bar complex of $\mathcal{A}$. (In [10], we typically worked with the unreduced bar complex $\operatorname{Bar}(\mathcal{A})$. The canonical inclusion $\operatorname{Bar}_{r}(\mathcal{A}) \rightarrow \operatorname{Bar}(\mathcal{A})$ is a homotopy equivalence.)

With this terminology in hand, we have the following reformulation of the complex of $\mathcal{A}_{\infty}$-module homomorphisms.

Proposition 2.10. For finite-dimensional right $\mathcal{A}_{\infty}$-modules $M$ and $N$, the chain complex of $\mathcal{A}_{\infty}$-module homomorphisms from $M$ to $N$, $\operatorname{Mor}_{\mathcal{A}}(M, N)$, is isomorphic to $N \otimes_{\mathcal{A}} \overline{\operatorname{Bar}_{r}(\mathcal{A})} \otimes_{\mathcal{A}} \bar{M}$.

Proof. Recall that an $\mathcal{A}_{\infty}$-morphism $f: M \rightarrow N$ consists of maps $f_{i+1}: M \otimes A[1]^{i}$ $\rightarrow N$. By unitality we can restrict the algebra inputs to lie in $A_{+}$, and because $M$ and $N$ are finite-dimensional, the space of such maps (as a vector space) is isomorphic to $N \otimes \operatorname{Hom}\left(T^{*}\left(A_{+}[1]\right), \mathbb{F}_{2}\right) \otimes \operatorname{Hom}\left(M, \mathbb{F}_{2}\right)$, which is the underlying space of $N \otimes_{\mathcal{A}}$ $\overline{\operatorname{Bar}_{r}(\mathcal{A})} \bigotimes_{\mathcal{A}} \bar{M}$. Checking that the differentials on the two complexes agree is again elementary.

A key property of the bar complex is that it can be used to give resolutions of modules. In the present language, this boils down to the following identity [10], Lemma 2.3.19:

$$
{ }^{\mathcal{A}} \mathrm{Bar}_{r}{ }^{\mathcal{A}} \otimes \mathcal{A} \simeq{ }^{\mathcal{A}}[\mathbb{I}]_{\mathcal{A}} .
$$

Here, ${ }^{\mathcal{A}}[\mathbb{I}]_{\mathcal{A}}$ is the type $D A$ bimodule whose modulification is $\mathcal{A}$; see [10], Definition 2.2.48. In particular, ${ }^{\mathcal{A}}[\mathbb{I}]_{\mathscr{A}}$ is the identity for $\otimes$.

We extend the definitions of duals to bimodules as follows. 
Definition 2.12. Let $\mathcal{A}$ and $\mathcal{B}$ be finite-dimensional $d g$ algebras over $\mathbb{k}=\bigoplus_{i=1}^{N} \mathbb{F}_{2}$ and $\mathrm{D}=\bigoplus_{i=1}^{M} \mathbb{F}_{2}$.

Suppose that ${ }^{\mathscr{A}, \mathscr{B}} M$ is a left-left type $D D$ module over $\mathcal{A}$ and $\mathscr{B}$. That is, ${ }^{\mathcal{A}, \mathscr{B}} M$

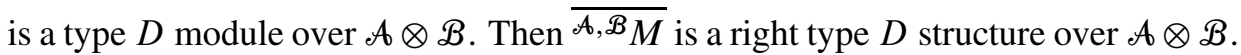
Interpreting this as a right-right type $D D$ structure $\bar{M}^{\mathcal{A}, \mathscr{B}}$, we call $\bar{M}^{\mathcal{A}, \mathscr{B}}$ the dual type DD structure to ${ }^{\mathcal{A}, \mathscr{B}} M$.

Now, suppose that $N_{\mathcal{A}, \mathscr{B}}$ is a right-right $\mathcal{A}_{\infty}$-bimodule over $\mathcal{A}$ and $\mathscr{B}$. Let $\bar{N}=$ $\operatorname{Hom}\left(M, \mathbb{F}_{2}\right)$. The transpose of the structure maps $m_{1, i, j}: N \otimes \mathcal{A}^{\otimes i} \otimes \mathcal{B}^{\otimes j} \rightarrow N$ are maps $m_{1, i, j}^{T}: \bar{N} \rightarrow \operatorname{Hom}\left(\mathscr{B}, \mathbb{F}_{2}\right)^{\otimes j} \otimes \operatorname{Hom}\left(\mathcal{A}, \mathbb{F}_{2}\right)^{\otimes i} \otimes \bar{N}$, which we interpret as maps

$$
\bar{m}_{j, i, 1}: \mathscr{B}^{\otimes j} \otimes \mathcal{A}^{\otimes i} \otimes \bar{N} \longrightarrow \bar{N} .
$$

We call $\left(\bar{N},\left\{\bar{m}_{j, i, 1}\right)\right.$ the dual $\mathcal{A}_{\infty}$-bimodule to $N_{\mathcal{A}, \mathcal{B}}$.

Finally, suppose ${ }^{\mathscr{A}} N_{\mathscr{B}}=\left(N, \delta^{1}\right)$ is a type $D A$ structure. Let $\bar{N}=\operatorname{Hom}\left(N, \mathbb{F}_{2}\right)$. The transpose of the map $\delta_{1+n}^{1}: N \otimes \mathcal{B}^{\otimes n} \rightarrow \mathcal{A} \otimes M$ is a map

$$
\left(\delta_{1+n}^{1}\right)^{T}: \bar{N} \otimes \operatorname{Hom}_{\mathbb{F}_{2}}\left(\mathcal{A}, \mathbb{F}_{2}\right) \longrightarrow \operatorname{Hom}_{\mathbb{F}_{2}}\left(\mathcal{B}, \mathbb{F}_{2}\right)^{\otimes n} \bar{N}
$$

We can interpret these as maps

$$
\bar{\delta}_{1+n}^{1}: \mathscr{B}^{\otimes n} \otimes \bar{N} \longrightarrow \bar{N} \otimes \mathcal{A} .
$$

We call $\left(\bar{N}, \bar{\delta}_{1+n}^{1}\right)$ the dual type DA structure to ${ }^{\mathcal{A}} N_{\mathcal{B}}$.

The following is an easy exercise.

Lemma 2.13. The dual type DD structure to a type DD structure satisfies the type $D D$ structure equation. The dual $\mathcal{A}_{\infty}$-bimodule to an $\mathcal{A}_{\infty}$-bimodule satisfies the $\mathcal{A}_{\infty}$-structure equation. The dual type DA structure to a DA structure satisfies the DA structure equation.

For bimodules, we can consider the space of morphisms over either one or both of the actions. So, Proposition 2.7 corresponds to two different statements for bimodules:

Proposition 2.14. Let $^{\mathcal{A}, \mathscr{B}} M$ and ${ }^{\mathcal{A}, \mathscr{B}} N$ be type DD structures, at least one of which is finite-dimensional. Then

$$
\operatorname{Mor}^{\mathcal{A} \otimes \mathscr{B}}(M, N) \cong \bar{M} \bigotimes_{\mathcal{A} \otimes \mathscr{B}}(\mathcal{A} \otimes \mathscr{B}) \bigotimes_{\mathcal{A} \otimes \mathscr{B}} N .
$$

Similarly, let ${ }^{\mathcal{A}, \mathscr{B}} M$ be a type $D D$ structure and let ${ }^{\mathscr{B}} N$ a type $D$ module. Then

$$
\operatorname{Mor}^{\mathscr{B}}(M, N) \cong \bar{M} \otimes_{\mathscr{B}} \mathfrak{B} \otimes_{\mathscr{B}} N,
$$

as type $D$ structures. A corresponding statement holds if $N$ is a type DD or DA module. 
Proof. Like Proposition 2.7, this is immediate from the definitions. (In fact, the first half also follows from Proposition 2.7.)

Lemma 2.15. Taking duals respects the operation $\otimes$ in the following sense: if $M_{\mathcal{A}}$ is an $\mathcal{A}_{\infty}$-module and ${ }^{\mathscr{A}} N$ is a type $D$ structure then

$$
\overline{M \otimes_{\mathcal{A}} N} \cong \bar{N} \otimes_{\mathscr{A}} \bar{M} .
$$

Moreover, if $M$ is an $\mathcal{A}_{\infty}$-bimodule or type DA structure and $N$ is a type $D A$ or DD structure, then the isomorphism in Equation (2.16) is an isomorphism of AA, DA, or DD structures (as appropriate).

Proof sketch. To get the structure maps on $\overline{M \otimes N}$, take the appropriate diagram from [10], Figure 4, rotate it $180^{\circ}$, and modify the diagram so all the algebra arrows point down. Then the module arrows are pointing up, as appropriate for diagrams involving the dual bimodule, and the diagrams are the same as those for the $\bar{N} \otimes \bar{M}$, as desired.

In this paper, we will pass freely between left modules over an algebra and right modules over the opposite algebra. Specifically, a right module $N_{\mathcal{A}}$ over $\mathcal{A}$ can be viewed as a left module over $\mathcal{A}^{\text {op }}$, which we write as ${ }_{\mathcal{A}^{\mathrm{op}}} N$, and a left type $D$ structure ${ }^{\mathcal{A}} M$ can be naturally viewed as a right type $D$ structure over $\mathcal{A}^{\text {op }}$, which we will write as $M^{\mathcal{A}^{\mathrm{op}}}$. The following is straightforward.

Lemma 2.17. There is an isomorphism

$$
\left(\mathcal{A} \otimes{ }^{\mathcal{A}} M\right)_{\mathcal{A}^{\mathrm{op}}} \cong\left(M^{\mathcal{A}^{\mathrm{op}}} \otimes \mathcal{A}^{\mathrm{op}}\right) .
$$

\section{3. $\alpha$ - $\beta$-bordered Heegaard diagrams}

3.1. $\boldsymbol{\beta}$ pointed matched circles. In [9] (as reviewed in Section 2.1), we gave a convention for how a pointed matched circle specifies a surface. In that paper, we considered exclusively the case where it was the $\alpha$-curves which ran out into the boundary. In the present paper, we will need to place the $\alpha$ - and $\beta$-curves on a more equal footing. Consequently, we would like to give a less biased construction, in the spirit of Zarev's work on sutured manifolds [22].

In this more symmetric construction, we make the following cosmetic revision to the notion of a pointed matched circle.

Definition 3.1. A decorated pointed matched circle consists of the following data:

- a circle $Z$;

- a decomposition of $Z$ into two closed oriented intervals, $Z_{\alpha}$ and $Z_{\beta}$, whose intersection consists of two points, and with $Z_{\alpha}$ and $Z_{\beta}$ oriented opposingly; 
- a collection of $4 k$ points $\mathbf{p}=\left\{p_{1}, \ldots, p_{4 k}\right\}$ in $Z$, so that either $\mathbf{p} \subset Z_{\alpha}$ or $\mathbf{p} \subset Z_{\beta}$

- a fixed-point-free involution $M$ on the points in $\mathbf{p}$; and

- a decoration by the letter $\alpha$ or the letter $\beta$, which indicates whether the points $\mathbf{p}$ lie in $Z_{\alpha}$ or $Z_{\beta}$.

We require that the points $\mathbf{p}$ and involution $M$ satisfy the condition that performing surgery on $Z$ along the zero-spheres specified by $\mathbf{p} / M$ gives a connected 1manifold. We abbreviate the data $\left(Z=Z_{\alpha} \cup Z_{\beta}, \mathbf{p}, M\right)$ by $\mathcal{Z}^{\alpha}$ or $Z^{\beta}$ (depending on the decoration); we will call the resulting object an $\alpha$-pointed matched circle or a $\beta$-pointed matched circle.

Construction 3.2. A decorated pointed matched circle $\mathbb{Z}^{\varepsilon}$ (where $\varepsilon$ is $\alpha$ or $\beta$ ) gives rise to a surface $F$ as follows. Consider the disk $D_{0}$ with boundary $Z$. We orient $D_{0}$ so that the specified orientation of $Z_{\alpha}$ agrees with its induced orientation from $\partial D_{0}$. Add a one-handle $s$ along the pair of points $\{h, t\}$; then add one-handles along all the $M$-matched points in $\mathbf{p}$; and finally attach two two-handles to fill the two remaining boundary components. Call the resulting surface $F\left(Z^{\varepsilon}\right)$. Each of the two disks attached at the last step meets exactly one of $Z_{\alpha}$ or $Z_{\beta}$ : we call these disks $D_{\alpha}$ and $D_{\beta}$, respectively. This surface has a preferred embedded disk $D_{\alpha} \cup s \cup D_{\beta}$, which is decomposed into three pieces. The surface inherits an orientation from $D_{0}$. (See Figure 1 for an illustration.)

The data of a decorated pointed matched circle $Z^{\alpha}$ is equivalent to the earlier data of a pointed matched circle: we contract the interval $Z^{\beta}$ to give the basepoint. The underlying surfaces can also be identified.
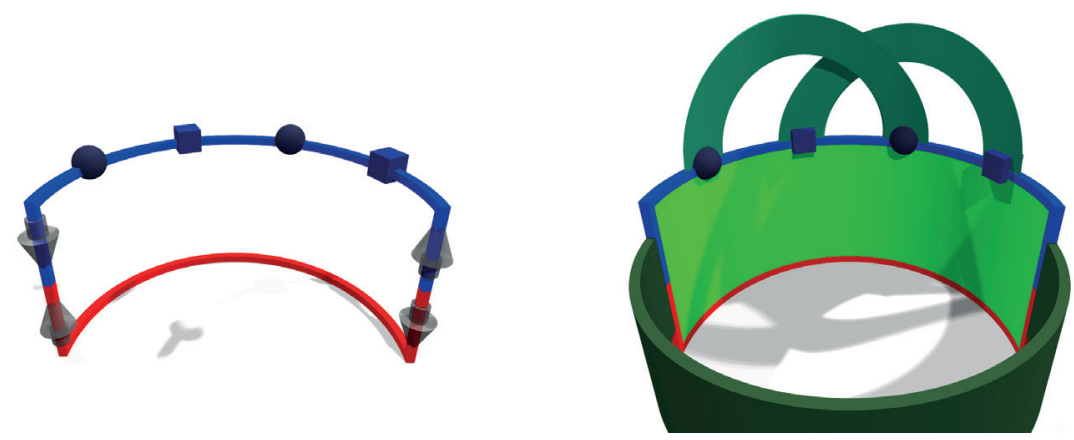

Figure 1. Constructing a surface from a $\beta$-pointed matched circle. Left: a $\beta$-pointed matched circle $Z^{\beta}$. The bottom half is the arc $Z_{\alpha}$ and the top half is the arc $Z_{\beta}$; translucent arrows indicate the orientations of $Z_{\alpha}$ and $Z_{\beta}$. Here, $k=1$, and the matching is indicated by the different shapes along the top. Right: part of the resulting surface $F\left(Z^{\beta}\right)$. The disk $D_{0}$ is in the back, and the 1-handle $s$ wraps around the front. $D_{\alpha}$ and $D_{\beta}$ are not shown. 
Definition 3.3. The strongly based $\alpha-\beta$ mapping class groupoid is the groupoid whose objects are decorated pointed matched circles. The morphisms from $Z_{1}^{\varepsilon_{1}}$ to $\mathcal{Z}_{2}^{\varepsilon_{2}}$ are isotopy classes of homeomorphisms

$$
\varphi: F\left(\mathcal{Z}_{1}^{\varepsilon_{1}}\right) \longrightarrow F\left(\mathcal{Z}_{2}^{\varepsilon_{2}}\right)
$$

so that

$$
\varphi\left(D_{\alpha}\left(\mathcal{Z}_{1}^{\varepsilon_{1}}\right)\right)=D_{\alpha}\left(\mathcal{Z}_{2}^{\varepsilon_{2}}\right), \quad \varphi\left(D_{\beta}\left(\mathcal{Z}_{1}^{\varepsilon_{1}}\right)\right)=D_{\beta}\left(\mathcal{Z}_{2}^{\varepsilon_{2}}\right), \quad \varphi\left(s\left(\mathcal{Z}_{1}\right)\right)=s\left(\mathcal{Z}_{2}\right) .
$$

In this paper, we sometimes consider homeomorphisms which are not in the mapping class groupoid as above. Specifically, we will sometimes be interested in homeomorphisms $\varphi: F\left(\mathcal{Z}_{1}^{\varepsilon}\right) \rightarrow F\left(\mathcal{Z}_{2}^{\varepsilon}\right)$ which preserve the preferred disk $D_{\alpha} \cup$ $s \cup D_{\beta}$, but which switch $D_{\alpha}$ and $D_{\beta}$. It is equivalent to consider the surface $F^{\circ}=F \backslash \operatorname{int}\left(D_{\alpha} \cup s \cup D_{\beta}\right)$, and think of the induced map on $F^{\circ}$, which exchanges $Z_{\alpha}=\left(\partial F^{\circ}\right) \cap D_{\alpha}$ and $Z_{\beta}=\left(\partial F^{\circ}\right) \cap D_{\beta}$.

One relevant class of such homeomorphisms is the following.

Definition 3.4. Let $Z^{\alpha}$ and $Z^{\beta}$ be two pointed matched circles which differ only in the $\alpha$ - or $\beta$-labels on the two intervals in the decomposition of $Z$. That is, $Z_{\alpha}$ for $Z^{\alpha}$ coincides with $Z_{\beta}$ for $\mathcal{Z}^{\beta}$. In this case, we say that $\mathcal{Z}^{\alpha}$ and $\mathcal{Z}^{\beta}$ are twin pointed matched circles. For twin pointed matched circles, there are canonical orientationreversing homeomorphisms

$$
K_{\alpha, \beta}: F\left(\mathcal{Z}^{\alpha}\right) \longrightarrow F\left(\mathcal{Z}^{\beta}\right), \quad K_{\beta, \alpha}: F\left(\mathcal{Z}^{\beta}\right) \longrightarrow F\left(\mathcal{Z}^{\alpha}\right) .
$$

Note that although these homeomorphisms send preferred disks to preferred disks, they do not preserve the decorations on those disks: $K_{\alpha, \beta}$ and $K_{\beta, \alpha}$ map $D_{\alpha}$ to $D_{\beta}$ and vice versa.

Another relevant class of such homeomorphisms are the "half boundary Dehn twists" defined below. Before defining them, we introduce some more terminology.

Definition 3.5. Let $A$ be an (oriented) annulus with one boundary component marked as the "inside boundary" and the other as the "outside boundary." A radial curve is any embedded curve in $A$ which connects the inside and outside boundary of $A$. Suppose that $r$ and $r^{\prime}$ are two oriented, radial curves which intersect the inside boundary of $A$ at the same point, but which are otherwise disjoint. We say that $r^{\prime}$ is to the right of $r$ if $r$ has a regular neighborhood $U$ with an orientation-preserving identification with $(-\varepsilon, \varepsilon) \times[0,1]$, so that $r$ is identified with $\{0\} \times[0,1]$, the inside boundary meets $U$ in $(-\varepsilon, \varepsilon) \times\{0\}$, and $r^{\prime} \cap U$ is contained in $[0, \varepsilon) \times[0,1]$.

Definition 3.6. Let $\left(F, D_{\alpha} \cup s \cup D_{\beta}\right)$ be a surface with a preferred disk decomposed into three parts. A positive half Dehn twist along the boundary, denoted $\tau_{\partial}^{1 / 2}$, is a homeomorphism with the following properties: 
- there is a disk neighborhood $N$ of $D_{\alpha} \cup s \cup D_{\beta}$ so that $\tau_{\partial}^{1 / 2}$ fixes the complement of $N$;

- $\tau_{\partial}^{1 / 2}$ maps the preferred disk to itself, but switches $D_{\alpha}$ and $D_{\beta}$; and

- there is a radial arc $r$ in the annulus $A=N \backslash \operatorname{int}\left(D_{\alpha} \cup s \cup D_{\beta}\right)$ (oriented so that it terminates at $\left.\partial\left(D_{\alpha} \cup s \cup D_{\beta}\right)\right)$ which is mapped under $\tau_{\partial}^{1 / 2}$ to a new arc $r^{\prime}$, which is to the right of $r$. Here, we view $\partial\left(D_{\alpha} \cup s \cup D_{\beta}\right)$ as the outside boundary of $A$.

See Figure 2 for an illustration. A negative half Dehn twist along the boundary, denoted $\tau_{\partial}^{-1 / 2}$, is the inverse to a positive half Dehn twist along the boundary. For a surface $F$ with one boundary component, the isotopy class of the surface homeomorphism specified by a half (positive or negative) Dehn twist (among homeomorphisms preserving the division of the disk) is uniquely specified by the above properties. Therefore we sometimes refer to "the" (rather than "a") positive (respectively negative) half Dehn twist along the boundary.

Similarly, a full Dehn twist along the boundary, $\tau_{\partial}$, is the composite of two positive half Dehn twists.

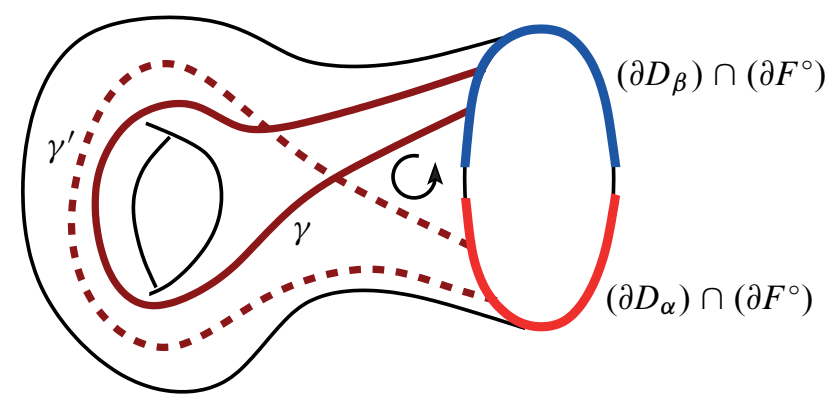

Figure 2. An example of a positive half Dehn twist along the boundary. The boundary of the genus one surface $F^{\circ}=F \backslash\left(D_{\alpha} \cup s \cup D_{\beta}\right)$ has a pair of distinguished $\operatorname{arcs}\left(\partial D_{\alpha} \cap \partial F^{\circ}\right) \subset Z_{\alpha}$ and $\left(\partial D_{\alpha} \cap \partial F^{\circ}\right) \subset Z_{\beta}$. We have illustrated an $\operatorname{arc} \gamma$ with boundary on $\partial D_{\beta}$ whose image is (up to isotopy relative to the boundary) the curve $\gamma^{\prime}$ represented by the dashed line.

Lemma 3.7. Let $f: F \rightarrow F$ be a homeomorphism preserving $D_{\alpha} \cup s \cup D_{\beta}$, and let $F^{\circ}=F \backslash\left(D_{\alpha} \cup s \cup D_{\beta}\right)$. Suppose that the following conditions hold.

(1) The induced automorphism $\bar{f}$ of the closed surface $F^{\circ} / \partial F^{\circ}$ is isotopic, relative to the basepoint $\left[\partial F^{\circ}\right]$, to the identity map.

(2) The map $f$ exchanges $D_{\alpha}$ and $D_{\beta}$.

(3) There is a curve $\gamma \subset F^{\circ}$ and a curve $\gamma^{\prime}$ isotopic relative to endpoints to $f(\gamma)$, so that:

(a) either the boundary of $\gamma$ is contained in $\partial D_{\beta} \cap \partial F^{\circ}$ or the boundary of $\gamma$ is contained in $\partial D_{\alpha} \cap \partial F^{\circ}$, 
(b) $[\gamma] \neq 0 \in H_{1}\left(F^{\circ}, \partial F^{\circ}\right)$,

(c) $\gamma$ intersects $\gamma^{\prime}$ transversely in exactly two points,

(d) $\gamma \cup \gamma^{\prime}$ has one component $T$ which is a disk, and

(e) in the cyclic order induced by the orientation on $F^{\circ}$, the boundary of $T$ consists of an arc in $\gamma$, an arc in $\gamma^{\prime}$, and an arc in $\partial F^{\circ}$.

(See Figure 2.) Then, $f$ is isotopic (as maps preserving $D_{\alpha} \cup s \cup D_{\beta}$ but switching $D_{\alpha}$ and $D_{\beta}$ ) to a positive half Dehn twist along the boundary.

Proof. Recall that the mapping class group of a surface $F$ fixing a disk $D \subset F$ is a $\mathbb{Z}$-central extension of the mapping class group of $F$ preserving $D$ set-wise (but not point-wise); and the Dehn twist $\tau_{\partial}$ along $\partial(F \backslash D)$ is a generator for this distinguished central $\mathbb{Z}$. Thus, Conditions (1) and (2) imply that $f$ is isotopic to $\tau_{\partial}^{m+1 / 2}$, for some $m \in \mathbb{Z}$. If $m \notin\{-1,0\}$ then the minimal number of intersection points between $\gamma$ and $f(\gamma)$ is greater than two, contradicting Condition (3c). The condition on the boundary of the triangle $T$ ensures that, in fact, $m$ is 0 , not -1 .

3.2. $\boldsymbol{\beta}$-bordered Heegaard diagrams. In previous work, [9] and [10], the $\alpha$-curves consisted of arcs and circles, while the $\beta$-curves were always circles. To prove the Hom pairing theorem, we will also want to work with diagrams where $\beta$ 's, instead of $\alpha$ 's, go out to the boundary.

Definition 3.8. A $\beta$-bordered Heegaard diagram is a quadruple $\mathscr{H}^{\beta}=(\bar{\Sigma}, \boldsymbol{\alpha}, \overline{\boldsymbol{\beta}}, \mathbf{z})$ where:

- $\bar{\Sigma}$ is a compact surface of genus $g$ with one boundary component;

- $\alpha$ is a $g$-tuple of pairwise disjoint circles in the interior $\Sigma$ of $\bar{\Sigma}$;

- $\overline{\boldsymbol{\beta}}$ is

$$
\overline{\boldsymbol{\beta}}=\{\overbrace{\bar{\beta}_{1}^{a}, \ldots, \bar{\beta}_{2 k}^{a}}^{\overline{\boldsymbol{\beta}}^{a}}, \overbrace{\beta_{1}^{c}, \ldots, \beta_{g-k}^{c}}^{\boldsymbol{\beta}^{c}},
$$

a collection of pairwise disjoint embedded arcs (the $\bar{\beta}_{i}^{a}$ ) with boundary on $\partial \bar{\Sigma}$ and circles (the $\beta_{i}^{c}$ ) in the interior $\Sigma$ of $\bar{\Sigma}$; and

- $\mathbf{z}$ is an arc in $\partial \bar{\Sigma} \backslash \overline{\boldsymbol{\beta}}^{a}$.

We require that $\Sigma \backslash \boldsymbol{\beta}$ and $\Sigma \backslash \boldsymbol{\alpha}$ both be connected; this translates to the condition that the $\beta$ - (respectively $\alpha$-) curves be linearly independent in $H_{1}(\bar{\Sigma}, \partial \bar{\Sigma})$.

The diagram $\mathscr{H}^{\beta}$ gives a natural $\beta$-pointed matched circle in the following way. Take two copies of $\partial \bar{\Sigma} \backslash \mathbf{z}$, call them $Z_{\alpha}$ and $Z_{\beta}$, and orient them both with the orientation they inherit from $\partial \bar{\Sigma}$. Let $Z$ be the result of gluing $Z_{\alpha}$ to $Z_{\beta}$ head-tohead and tail-to-tail. Let $\mathbf{p}=\overline{\boldsymbol{\beta}}^{a} \cap \partial \bar{\Sigma}$, thought of as a subset of $Z_{\beta}$. Let $M$ be the involution exchanging the endpoints of each $\bar{\beta}_{i}^{a}$. We call this pointed matched circle $\mathcal{Z}\left(\mathscr{H}^{\beta}\right)$. 
We will sometimes call bordered Heegaard diagrams as defined in [9] $\alpha$-bordered Heegaard diagrams, and denote them $\mathscr{H}^{\alpha}$, to distinguish them from $\beta$-bordered Heegaard diagrams. Note that we have a slight shift in point of view from [9]: we now think of $\mathbf{z}$ as an interval, rather than just a point $z$, and our circle $Z$ is no longer $\partial \bar{\Sigma}$, but rather two copies of an interval in $\bar{\Sigma}$. An $\alpha$-bordered Heegaard diagram specifies an $\alpha$-pointed matched circle in exactly the same way as a $\beta$-bordered Heegaard diagram specifies a $\beta$-pointed matched circle, but placing the points in $Z_{\alpha}$ rather than $Z_{\beta}$.

Construction 3.9. Let $Z^{\beta}$ denote the pointed matched circle specified by $\mathscr{H}^{\beta}$. There is an associated bordered three-manifold $Y\left(\mathscr{H}^{\beta}\right)$, constructed in the following four steps; see Figure 3.

(1) Glue $[0,1] \times \bar{\Sigma}$ to $[0,1] \times F\left(\mathcal{Z}^{\beta}\right)$, by identifying $[0,1] \times(\partial \bar{\Sigma})$ with $\{0\} \times\left(D_{0} \cup s\right)$ so that

- $(\mathbf{z} \subset \partial \bar{\Sigma}) \times[0,1]$ is identified with the one-handle $\{0\} \times\left(s \subset F\left(Z^{\beta}\right)\right)$,

- $[0,1] \times(\partial \bar{\Sigma} \backslash \mathbf{z})$ is identified with $\{0\} \times D_{0}$, and

- $\{1\} \times \mathbf{p} \subset[0,1] \times \bar{\Sigma}$ is identified with $\{0\} \times \mathbf{p} \subset[0,1] \times F\left(Z^{\beta}\right)$.

(The result is naturally a manifold with corners.)

(2) Attach 3-dimensional 2-handles to the $\{0\} \times \alpha_{i} \subset[0,1] \times \bar{\Sigma}$ and to the $\{1\} \times \beta_{i}^{c} \subset$ $[0,1] \times \bar{\Sigma}$.

(3) Let $\eta_{i}$ denote the core of the 1-handle in $F\left(\mathcal{Z}^{\beta}\right)$ attached along $\beta_{i}^{a} \cap \partial \bar{\Sigma}$. Then $\left(\{1\} \times \beta_{i}^{a}\right) \cup\left(\{0\} \times \eta_{i}\right)$ is a closed circle; attach thickened disks (3-dimensional 2-handles) along these circles.

The result of the attaching so far is a 3-manifold with three boundary components: two copies of $S^{2}$ (one containing $D_{\alpha}$ and the other containing $D_{\beta}$ ) and a copy of $F\left(Z^{\beta}\right)$.

(4) Fill in the two $S^{2}$ boundary components with 3-balls.

The boundary of $Y\left(\mathscr{H}^{\beta}\right)$ is naturally (orientation-preserving) identified with $F\left(Z^{\beta}\right)$.

Remark 3.10. The construction of $Y\left(\mathscr{H}^{\beta}\right)$ is convenient for describing the effect of gluing Heegaard diagrams. For the $\alpha$-bordered case (which is exactly analogous), a shorter description of an equivalent bordered three-manifold is given in [10], Construction 5.3; see also Construction 3.19 below. In particular, this construction identifies a regular neighborhood of the $\alpha$-arcs union the boundary in $\mathscr{H}^{\alpha}$ with $F^{\circ}(\partial \mathscr{H})$, in an orientation-reversing way. By contrast, for a $\beta$-bordered Heegaard diagram, the corresponding identification is orientation-preserving. 

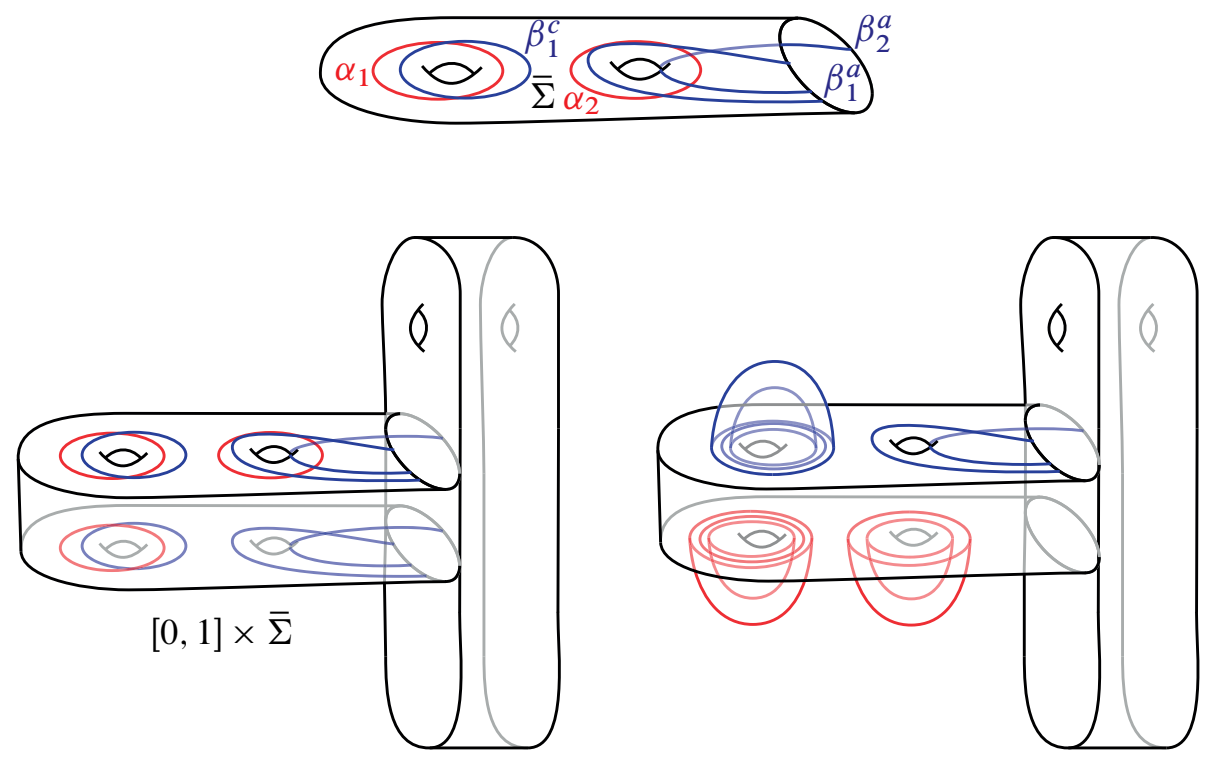

$[0,1] \times F\left(\mathcal{Z}^{\beta}\right)$
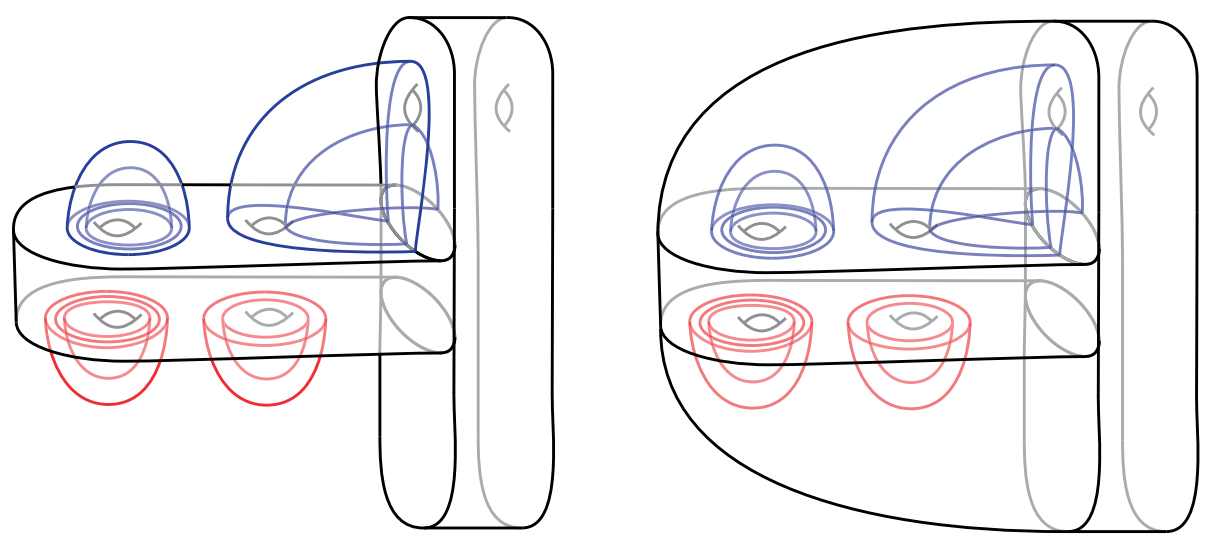

Figure 3. Building a bordered 3-manifold from a $\boldsymbol{\beta}$-bordered Heegaard diagram. The analogous figure in the $\alpha$-bordered case is [9], Figure 4.1. 
We now wish to define $\widehat{\mathrm{CFD}}\left(\mathscr{H}^{\beta}\right)$ and $\widehat{\mathrm{CFA}}\left(\mathscr{H}^{\beta}\right)$. As for $\alpha$-bordered diagrams, these will be defined by counting moduli spaces $\mathcal{M}^{B}(\mathbf{x}, \mathbf{y} ; \overrightarrow{\boldsymbol{\rho}})$ between two generators $\mathbf{x}$ and $\mathbf{y}$ asymptotic to certain sequences of sets of Reeb chords on $\mathcal{Z}^{\beta}$; see [9], Definition 5.61. However, the fact the diagram is $\beta$-bordered leads to some reversals. It is easiest to see what happens by reference to a corresponding $\alpha$-bordered diagram.

Definition 3.11. Given an $\alpha$-bordered Heegaard diagram $\mathscr{H}^{\alpha}=(\Sigma, \boldsymbol{\alpha}, \boldsymbol{\beta}, \mathbf{z})$ there is an associated $\beta$-bordered Heegaard diagram $\overline{\mathscr{H}}^{\beta}=\left(\Sigma, \boldsymbol{\alpha}^{\beta}, \boldsymbol{\beta}^{\beta}, \mathbf{z}\right)$ obtained by $\operatorname{setting} \beta_{i}^{\beta, c}=\alpha_{i}^{c}, \beta_{i}^{\beta, a}=\alpha_{i}^{a}, \alpha_{i}^{\beta}=\beta_{i}$.

Lemma 3.12. Let $Y$ be a 3-manifold and $\varphi: F\left(Z^{\alpha}\right) \rightarrow \partial Y$ a parameterization of its boundary. Let $\mathscr{H}^{\alpha}$ be an $\alpha$-bordered Heegaard diagram for $(Y, \varphi)$. Then $\overline{\mathscr{H}}^{\beta}$ is a $\beta$-bordered Heegaard diagram for $\left(-Y, \varphi \circ K_{\beta, \alpha}: F\left(Z^{\beta}\right) \rightarrow-\partial Y\right)$.

Proof. Recall from [9], Construction 4.6, that if $\mathscr{H}^{\alpha}$ is an $\alpha$-bordered Heegaard diagram then to construct $Y\left(\mathscr{H}^{\alpha}\right)$ one thickens $\bar{\Sigma}$ to $\bar{\Sigma} \times[0,1]$; glues the boundary $(\partial \bar{\Sigma}) \times[0,1]$ to $\left(D_{0} \cup s\right) \subset F\left(\mathcal{Z}^{\alpha}\right)$, and then one glues thickened disks to the following objects:

- the $\alpha$-circles in $\bar{\Sigma} \times\{0\}$,

- $\beta$-circles in $\bar{\Sigma} \times\{1\}$, and

- the unions of the $\alpha$-arcs in $\bar{\Sigma} \times\{0\}$ and the cores of the 1-handles of $F\left(\mathcal{Z}^{\alpha}\right)$.

Finally, one caps off the two $S^{2}$ boundary components with 3-balls.

This process results in a manifold $Y\left(\mathscr{H}^{\alpha}\right)$ which is the mirror image, in an obvious sense, of the manifold $Y\left(\overline{\mathscr{H}}^{\beta}\right)$ from Construction 3.9; reflecting across $\Sigma \times\{1 / 2\}$ gives an orientation-reversing homeomorphism between the two bordered manifolds.

For each generator $\mathbf{x}$ of $\mathscr{H}^{\alpha}$, there is an obvious corresponding generator $\overline{\mathbf{x}}$ of $\overline{\mathscr{H}}^{\beta}$. Similarly, for a homology class $B \in \pi_{2}(\mathbf{x}, \mathbf{y})$ let $\bar{B} \in \pi_{2}(\overline{\mathbf{y}}, \overline{\mathbf{x}})$ denote the homology class with the same local multiplicities in $\Sigma$ as $B$.

Lemma 3.13. For $\mathscr{H}$ an $\alpha$-bordered Heegaard diagram, $\mathbf{x}$ and $\mathbf{y}$ generators of $\mathcal{H}$, $B \in \pi_{2}(\mathbf{x}, \mathbf{y})$, and $\vec{\rho}$ any sequence of sets of Reeb chords, there is a homeomorphism

$$
\mathcal{M}^{B}(\mathbf{x}, \mathbf{y} ; \overrightarrow{\boldsymbol{\rho}}) \cong \mathcal{M}^{\bar{B}}\left(\overline{\mathbf{y}}, \overline{\mathbf{x}}, \overrightarrow{\boldsymbol{\rho}}^{\mathrm{op}}\right)
$$

where $\overrightarrow{\boldsymbol{\rho}}^{\mathrm{op}}$ is $\overrightarrow{\boldsymbol{\rho}}$ read in the opposite order.

Proof. Both moduli spaces are defined by counting pseudoholomorphic curves in $\Sigma \times[0,1] \times \mathbb{R}$. Reflecting in both the $[0,1]$ and $\mathbb{R}$ directions gives an identification between the two moduli spaces. 
For a $\beta$-bordered Heegaard diagram $\mathscr{H}^{\beta}$, we can therefore use all the techniques of [9] to define $\widehat{\mathrm{CFD}}\left(\mathscr{H}^{\beta}\right)$ and $\widehat{\mathrm{CFA}}\left(\mathscr{H}^{\beta}\right)$, except that the order of Reeb chords on the boundary is reversed. We can achieve this algebraically either by reversing all the chords or (as we prefer) by viewing our modules as defined over the opposite algebra. (These are equivalent, by Equation (2.1).) Thus we view $\widehat{\mathrm{CFD}}\left(\mathscr{H}^{\beta}\right)$ as a (left) type $D$ structure over $\mathcal{A}(-\mathcal{Z})^{\text {op }}$, i.e. a right type $D$ structure over $\mathcal{A}(-\mathcal{Z})$, and $\widehat{\mathrm{CFA}}\left(\mathscr{H}^{\beta}\right)$ as a left $\mathcal{A}_{\infty}$-module over $\mathcal{A}(\mathcal{Z})$.

We digress briefly to discuss the identification between $\operatorname{spin}^{c}$-structures on $Y$ and $-Y$. As usual, we view a spin ${ }^{c}$-structure on $Y$ as a homology class of non-vanishing vector fields. Given a $\operatorname{spin}^{c}$-structure $\mathfrak{s}$ on $Y$, induced by a vector field $v$, let $-\mathfrak{s}$ be the $\operatorname{spin}^{c}$-structure on $-Y$ induced by the vector field $-v$. (Note that the oriented 2-plane fields $v^{\perp}$ on $Y$ and $(-v)^{\perp}$ on $-Y$ are the same.) To avoid confusion, recall that the conjugate $\operatorname{spin}^{c}$-structure $\overline{\mathfrak{s}}$ is also represented by $-v$, but viewed as a vector field on $Y$. Thus, $-\overline{\mathfrak{s}}$ is represented by $v$ as a vector field on $-Y$.

Proposition 3.14. Let $\mathscr{H}^{\alpha}$ be an $\alpha$-bordered Heegaard diagram with boundary $\mathcal{Z}=$ $Z^{\alpha}$. Then $\widehat{\mathrm{CFD}}\left(\overline{\mathscr{H}}^{\beta}\right)$ and $\widehat{\mathrm{CFA}}\left(\overline{\mathcal{H}}^{\beta}\right)$ are duals (in the senses of Definitions 2.5 and 2.8 ) of the corresponding structures for $\mathscr{H}^{\alpha}$ :

$$
\begin{aligned}
\widehat{\mathrm{CFD}}\left(\overline{\mathscr{H}}^{\beta},-\mathfrak{s}\right)^{\mathcal{A}(-Z)} \cong \mathcal{A A}^{(-Z)} \overline{\widehat{\mathrm{CFD}}\left(\mathscr{H}^{\alpha}, \mathfrak{s}\right)}, \\
\mathcal{A}(\mathcal{Z}) \widehat{\mathrm{CFA}}\left(\overline{\mathscr{H}}^{\beta},-\mathfrak{s}\right) \cong \overline{\widehat{\mathrm{CFA}}\left(\mathscr{H}^{\alpha}, \mathfrak{s}\right)} \\
\mathcal{A}(\mathcal{Z})
\end{aligned}
$$

Proof. We will prove the duality result for $\widehat{\mathrm{CFD}}$; the proof for $\widehat{\mathrm{CFA}}$ is analogous. Let $X\left(\mathscr{H}^{\alpha}\right)$ (respectively $X\left(\overline{\mathscr{H}}^{\beta}\right)$ ) denote the $\mathbb{k}$-module generated by $\mathfrak{S}\left(\mathscr{H}^{\alpha}\right)$ (respectively $\left.\mathfrak{S}\left(\overline{\mathscr{H}}^{\beta}\right)\right)$. We have an isomorphism $X\left(\overline{\mathscr{H}}^{\beta}\right)=\operatorname{Hom}\left(X\left(\mathscr{H}^{\alpha}\right)\right.$, $\left.\mathbb{k}\right)$ by setting, for generators $\mathbf{x}, \mathbf{y} \in \mathfrak{S}\left(\mathscr{H}^{\alpha}\right)$,

$$
\overline{\mathbf{x}}(\mathbf{y})= \begin{cases}\iota_{\mathbf{x}} & \text { if } \mathbf{x}=\mathbf{y}, \\ 0 & \text { otherwise }\end{cases}
$$

where $l_{\mathbf{x}} \in \mathbb{k}$ is the primitive idempotent corresponding to $\mathbf{x}$, so $l_{\mathbf{x}} \mathbf{x}=\mathbf{x}$.

The type $D$ structure on $\widehat{\mathrm{CFD}}\left(\mathscr{H}^{\alpha}\right)$ is given by

$$
\begin{aligned}
& \delta^{1}: X\left(\mathcal{H}^{\alpha}\right) \longrightarrow \mathcal{A}(-\mathcal{Z}) \otimes X\left(\mathscr{H}^{\alpha}\right), \\
& \delta^{1}(\mathbf{x})=\sum_{\mathbf{y}} \sum_{\substack{\vec{\beta} \in \in \pi_{2}(\mathbf{x}, \mathbf{y}) \\
\text { compatithe with } B \\
\text { ind }(B, \vec{\rho})=1}} a(-\vec{\rho}) \otimes \mathbf{y} \cdot \#\left(\mathcal{M}^{B}(\mathbf{x}, \mathbf{y} ; \vec{\rho})\right) .
\end{aligned}
$$

The operation $\delta_{\beta}^{1}: X\left(\overline{\mathscr{H}}^{\beta}\right) \rightarrow X\left(\overline{\mathscr{H}}^{\beta}\right) \otimes \mathcal{A}(-\mathcal{Z})$ is defined similarly, but using the moduli spaces on $\overline{\mathscr{H}}^{\beta}$. By Lemma 3.13 , terms of the form $a(-\vec{\rho}) \otimes \mathbf{y}$ in $\delta^{1}(\mathbf{x})$ 
correspond to terms of the form $\overline{\mathbf{x}} \otimes a(-\vec{\rho})$ in $\delta_{\beta}^{1}(\overline{\mathbf{y}})$. (We are considering the moduli space in which the chords appear in reverse order, but we also multiply the algebra elements in the reverse order, so it is again $a(-\vec{\rho})$ that is relevant.) This is exactly the statement that $\widehat{\mathrm{CFD}}\left(\overline{\mathscr{H}}^{\beta}\right)$ is the dual of $\widehat{\mathrm{CFD}}\left(\mathscr{H}^{\alpha}\right)$.

The behavior on the $\operatorname{spin}^{c}$ structures comes from the observation that, when $\mathbf{x}$ is viewed as a generator for the bordered Floer homology of $\mathscr{H}$, its corresponding vector field points in the opposite direction from that of $\overline{\mathbf{x}}$ when viewed as a generator for the bordered Floer homology of $\overline{\mathscr{H}}$.

Note that $\overline{\mathscr{H}}^{\beta}$ represents $-Y\left(\mathscr{H}^{\alpha}\right)$. There is another way of creating a Heegaard diagram for $-Y\left(\mathscr{H}^{\alpha}\right)$, namely by considering $-\mathscr{H}^{\alpha}$, which is the same Heegaard diagram but with the orientation on the underlying surface $\Sigma$ reversed. This operation also has the effect of dualizing modules:

Proposition 3.15. Suppose that $\mathscr{H}$ is an $\alpha$-bordered Heegaard diagram with boundary Z. Let $-\mathscr{H}$ denote the same Heegaard diagram but with the orientation of $\Sigma$ reversed. Then $\widehat{\mathrm{CFD}}(-\mathcal{H})$ is a left $\mathcal{A}(\mathcal{Z})$-module. If we view $\widehat{\mathrm{CFD}}(-\mathscr{H})$ as a right $\mathcal{A}(-\mathcal{Z})$-module, then $\widehat{\mathrm{CFD}}(-\mathcal{H},-\overline{\mathfrak{s}})$ is dual to $\widehat{\mathrm{CFD}}(\mathscr{H}, \mathfrak{s})$. Similarly, $\widehat{\mathrm{CFA}}(-\mathcal{H},-\overline{\mathfrak{s}})$ is dual to $\widehat{\mathrm{CFA}}(\mathcal{H}, \mathfrak{s})$.

Proof. This follows from a similar argument to Proposition 3.14.

3.3. $\boldsymbol{\alpha}-\boldsymbol{\beta}$-bordered Heegaard diagrams. We will need to generalize the notion of arced bordered Heegaard diagrams with two components and the associated 3-manifolds to cases where the boundaries meet $\alpha$ - or $\beta$-circles. The following is an extension of [10], Definition 5.1, to our more symmetric language.

Definition 3.16. A strongly bordered three-manifold with two boundary components is specified by the following data:

- A 3-manifold $Y$ with two boundary components $\partial_{L} Y$ and $\partial_{R} Y$,

- homeomorphisms $\varphi_{L}: F\left(\mathcal{Z}_{L}^{\varepsilon_{L}}\right) \rightarrow \partial_{L} Y$ and $\varphi_{R}: F\left(\mathcal{Z}_{R}^{\varepsilon_{R}}\right) \rightarrow \partial_{R} Y$ for some pointed matched circles $\mathcal{Z}_{L}^{\varepsilon_{L}}$ and $\mathfrak{Z}_{R}^{\varepsilon_{R}}$, both preserving orientation, and

- a tunnel $[0,1] \times D$ connecting the two boundary components, which is divided into three balls

$$
\left([0,1] \times D_{\alpha}\right) \cup([0,1] \times s) \cup\left([0,1] \times D_{\beta}\right)
$$

where $\{0\} \times\left(D_{\alpha} \cup s \cup D_{\beta}\right)$ coincides with the corresponding part of $\partial_{L} Y=$ $\varphi_{L}\left(F\left(Z_{L}^{\varepsilon_{L}}\right)\right)$ and $\{1\} \times\left(D_{\alpha} \cup s \cup D_{\beta}\right)$ coincides with the corresponding part of $\partial_{R} Y=\varphi_{R}\left(F\left(\mathcal{Z}_{R}^{\varepsilon R}\right)\right)$.

For 3-manifolds with two boundary components, one can consider arced $(\alpha, \alpha)$ bordered Heegaard diagrams (which are the only kind we considered in [10]), arced 
$(\alpha, \beta)$-bordered Heegaard diagrams, arced $(\beta, \alpha)$-bordered Heegaard diagrams, and arced $(\beta, \beta)$-bordered Heegaard diagrams. We call all of these types of diagrams, collectively, arced bordered Heegaard diagrams. The definitions of the latter three types are trivial adaptations of the definition of an arced bordered Heegaard diagram from [10], Section 5; except that in keeping with our present conventions (where we thicken basepoints), rather than drawing simply an arc connecting the two basepoints on the boundary, now $\mathbf{z}$ denotes a rectangle in the Heegaard surface which connects the arc of basepoints on one boundary component with the arc of basepoints in the other. For $(\beta, \beta)$-bordered Heegaard diagrams the $\beta$-curves but not the $\alpha$-curves go out to the boundaries. For $(\alpha, \beta)$-bordered diagrams, the $\alpha$-curves go out to $\partial_{L} \bar{\Sigma}$ and the $\beta$-curves go out to $\partial_{R} \bar{\Sigma}$. For $(\beta, \alpha)$-bordered Heegaard diagrams, the $\beta$-curves go out to $\partial_{L} \bar{\Sigma}$ and the $\alpha$-curves go out to $\partial_{R} \bar{\Sigma}$.

More explicitly, if $\mathscr{H}$ is an arced bordered Heegaard diagram, then there are two intervals $Z_{L}$ and $Z_{R}$ in $\partial \bar{\Sigma}$, with the property that $\mathbf{p}=(\partial \bar{\Sigma}) \cap\left(\overline{\boldsymbol{\alpha}}^{a} \cup \overline{\boldsymbol{\beta}}^{a}\right)$ is contained in $Z_{L} \cup Z_{R}$, so that all points $\mathbf{p} \cap Z_{L}$ are all of the same type (i.e. all are either boundary points of $\alpha$-arcs or $\beta$-arcs) and $\mathbf{p} \cap Z_{R}$ are also of the same type. Correspondingly we let $\partial_{L} \mathscr{H}$ denote the pointed matched circle gotten by doubling $Z_{L}$ and marking it with $\alpha$ or $\beta$, according to the type of points in $\mathbf{p} \cap Z_{L}$, and we obtain $\partial_{R} \mathscr{H}$ analogously.

An arced bordered diagram gives rise to a strongly bordered three-manifold via the following generalization of Construction 3.9.

Construction 3.17. Let $Z_{L}$ and $Z_{R}$ denote the pointed matched circles specified by $\partial_{L} \mathscr{H}$ and $\partial_{R} \mathscr{H}$. There is an associated strongly bordered three-manifold $Y(\mathscr{H})$ with two boundary components constructed in the following four steps.

(1) Glue $[0,1] \times \bar{\Sigma}$ to $[0,1] \times F\left(\mathcal{Z}_{L}\right)$, by gluing $[0,1] \times \partial_{L} \bar{\Sigma}$ to $\{0\} \times F\left(\mathcal{Z}_{L}\right)$ and $[0,1] \times \partial_{R} \bar{\Sigma}$ to $\{0\} \times F\left(Z_{R}\right)$ following Construction 3.9.

(2) Next, glue 2-handles along $\{0\} \times \alpha_{i}^{c} \subset \overline{[}[0,1] \times \Sigma$ and to $\{1\} \times \beta_{i}^{c} \subset[0,1] \times \bar{\Sigma}$.

(3) Consider the $\operatorname{arcs} \alpha_{i}^{a}$ and $\beta_{i}^{a}$, thought of as supported in $\{0\} \times \bar{\Sigma}$ and $\{1\} \times \bar{\Sigma}$ respectively. These are completed into closed circles by following the endpoints through the one-handles in $F\left(Z_{L}\right)$ or $F\left(Z_{R}\right)$ (wherever they go). Add twohandles along these circles.

We end up with a three-manifold with four boundary components: $F\left(\mathcal{Z}_{L}\right)$, $F\left(Z_{R}\right)$ and a pair of two-spheres.

(4) Fill in the two-spheres with three-balls $B_{\alpha}$ and $B_{\beta}$, to obtain the desired threemanifold $Y(\mathscr{H})$.

Morally, the tunnel $[0,1] \times D$ needed to make $Y(\mathscr{H})$ into a strongly bordered 3-manifold is given by $B_{\alpha} \cup[0,1] \times \mathbf{z} \cup B_{\beta}$. In fact, since we have attached copies of $[0,1] \times F\left(\mathcal{Z}_{L}\right)$ and $[0,1] \times F\left(Z_{R}\right)$ at the boundary, this tunnel is actually given by

$$
\begin{aligned}
\left(\left([0,1] \times D_{\alpha}\right) \cup B_{\alpha} \cup\left([0,1] \times D_{\alpha}\right)\right) & \cup(([0,1] \times s) \cup([0,1] \times \mathbf{z}) \cup([0,1] \times s)) \\
& \cup\left(\left([0,1] \times D_{\beta}\right) \cup B_{\beta} \cup\left([0,1] \times D_{\beta}\right)\right) .
\end{aligned}
$$


Lemma 3.18. If $\mathscr{H}$ and $\mathscr{H}^{\prime}$ are two arced bordered Heegaard diagrams with $\partial_{R} \mathscr{H}=$ $-\partial_{L} \mathscr{H}^{\prime}=\mathcal{Z}$ (agreeing as pointed matched circles, including decoration), then

$$
Y\left(\mathscr{H}_{\partial_{R}} \cup_{\partial_{L}} \mathscr{H}^{\prime}\right)=Y(\mathscr{H}) \cup_{F(\mathcal{Z})} Y\left(\mathscr{H}^{\prime}\right) .
$$

Proof. This is immediate from the definitions.

We give the following simpler description in the $\alpha-\beta$-bordered case which will be used in the proof of Proposition 4.2.

Construction 3.19. An arced $(\alpha, \beta)$-bordered Heegaard diagram $\mathcal{H}$ specifies a strongly bordered three-manifold as follows. Attach disks $D_{L}$ and $D_{R}$ to $\partial_{L} \bar{\Sigma}$ and $\partial_{R} \bar{\Sigma}$ respectively obtain a closed surface $\Sigma$. Let $Y$ be $[0,1] \times \Sigma$ with three-dimensional two-handles attached along the $\{0\} \times \alpha_{i}^{c}$ and $\{1\} \times \beta_{i}^{c}$.

$F\left(\mathcal{Z}_{L}\right)$ is identified with $\partial_{L} Y$ as follows.

- Identify $s$ with $\{0\} \times \mathbf{z}$.

- Identify $D_{\beta}$ with $\{0\} \times D_{L}$.

- Identify $D_{\alpha}$ with $\{0\} \times D_{R}$.

- Identify $F^{\circ}\left(\mathcal{Z}_{L}\right)$ with the complement of $\{0\} \times D_{L} \cup\{0\} \times \mathbf{z} \cup\{0\} \times D_{R} \subset \partial_{L} Y$ using the $\alpha$-arcs.

$F\left(Z_{R}\right)$ is identified with $\partial_{R} Y$ as follows.

- Identify $s$ with $\{1\} \times \mathbf{z}$.

- Identify $D_{\beta}$ with $\{1\} \times D_{L}$.

- Identify $D_{\alpha}$ with $\{1\} \times D_{R}$

- Identify $F^{\circ}\left(\mathcal{Z}_{R}\right)$ with the complement of $\{1\} \times D_{L} \cup\{1\} \times \mathbf{z} \cup\{1\} \times D_{R} \subset \partial_{L} Y$ using the $\beta$-arcs.

The tunnel is, of course, $[0,1] \times\left(D_{L} \cup \mathbf{z} \cup D_{R}\right)$.

Lemma 3.20. If $\mathscr{H}$ is an $\alpha$ - $\beta$-bordered diagram, the strongly bordered three-manifolds specified in Constructions 3.19 and 3.17 are canonically isomorphic.

Proof. Let $Y_{1}(\mathscr{H})$ (respectively $Y_{2}(\mathscr{H})$ ) be the strongly bordered 3-manifold given by Construction 3.17 (respectively Construction 3.19). Observe that $Y_{2}(\mathscr{H})$ is a subspace of $Y_{1}(\mathscr{H})$ in an obvious way. The two-handles attached in Step (3) of Construction 3.17 specify a deformation retraction of $Y_{1}(\mathscr{H})$ to $Y_{2}(\mathscr{H})$ (by folding the boundary along the 2-handles to the Heegaard surface), respecting the strong bordering. 
Construction 3.21. Let $Y$ be a strongly bordered 3-manifold with boundary components parameterized by $F\left(\mathcal{Z}_{L}\right)$ and $F\left(\mathcal{Z}_{R}\right)$. Suppose that $Y$ is homeomorphic to the product of an interval with a surface. Then we can define a map

$$
\varphi_{Y}:-F\left(\mathcal{Z}_{L}\right) \longrightarrow F\left(\mathcal{Z}_{R}\right)
$$

in the strongly based mapping class groupoid as follows.

First, fix a homeomorphism $\Phi:[0,1] \times F\left(\mathcal{Z}_{R}\right) \rightarrow Y$ so that

- $\left.\Phi\right|_{\{1\} \times F\left(\mathcal{Z}_{R}\right)}=\varphi_{R}$ and

- the images under $\Phi$ of $[0,1]$ times the three distinguished regions $D_{\alpha}, s$, and $D_{\beta}$ (in $F\left(\mathcal{Z}_{R}\right)$ ) are mapped to the three corresponding distinguished regions in $Y$.

Then, let

$$
\varphi_{Y}=\left(\left.\Phi\right|_{\{0\} \times F\left(\mathcal{Z}_{R}\right)}\right)^{-1} \circ\left(-\varphi_{L}\right): F\left(\mathcal{Z}_{L}\right) \longrightarrow F\left(\mathcal{Z}_{R}\right) .
$$

We call the strongly bordered manifold $Y$ the mapping cylinder of $\varphi$.

An adaptation of the argument from [10], Lemma 5.29, shows that the above construction gives a well-defined element of the mapping class groupoid in the sense of Definition 3.3.

Definition 3.22. Given a strongly based homeomorphism $\varphi: F\left(Z_{1}\right) \rightarrow F\left(Z_{2}\right)$ and a bordered 3-manifold $\left(Y, \psi: F\left(\mathcal{Z}_{1}\right) \rightarrow \partial Y\right)$ we can twist the parameterization of $Y$ by $\varphi$ to give a new bordered 3-manifold

$$
\varphi(Y)=\left(Y, \psi \circ \varphi^{-1}: F\left(Z_{2}\right) \longrightarrow \partial Y\right) .
$$

Equivalently, we can define $\varphi(Y)$ by gluing the mapping cylinder of $\varphi$ to $Y$ :

$$
\varphi(Y)=Y \cup_{F\left(Z_{1}\right)} M_{\varphi}
$$

For arced bordered Heegaard diagrams, one can define bimodules as in [10]. The case of $(\alpha, \alpha)$-bordered diagrams is discussed there, and the story for $(\beta, \beta)$-bordered diagrams is an entirely straightforward adaptation (although with reversed algebras), analogous to the relation between $\alpha$-bordered and $\beta$-bordered diagrams as discussed in Section 3.2. Some aspects of the $(\beta, \alpha)$-bordered case are new, however, and we discuss them now.

For a $(\beta, \alpha)$-bordered Heegaard diagram ${ }^{\beta} \mathcal{H}^{\alpha}$, a generator is a tuple $\mathbf{x}=\left\{x_{i}\right\}$ of intersection points between $\alpha$-and $\beta$-curves, such that there is exactly one $x_{i}$ on each $\alpha$ - or $\beta$-circle, and no $\alpha$ - or $\beta$-curve contains more than one $x_{i}$. Note that, unlike the case of an $(\alpha, \alpha)$ - or $(\beta, \beta)$-bordered Heegaard diagram, for a fixed diagram ${ }^{\beta} \mathcal{H}^{\alpha}$, different generators can have different cardinalities.

The type $A A$ module $\widehat{\mathrm{CFAA}}\left({ }^{\beta} \mathcal{H}^{\alpha}\right)$ associated to ${ }^{\beta} \mathcal{H}^{\alpha}$ is generated over $\mathbb{k}$ by the generators $\mathbf{x}$. The boundary of $\beta_{\mathcal{H}^{\alpha}}$ consists of pointed matched circles $Z_{L}^{\beta}$ (coming from the $\beta$-arcs) and $Z_{R}^{\alpha}$ (coming from the $\alpha$-arcs). We define an action of the 
idempotents of $\mathcal{A}\left(\mathcal{Z}_{L}\right)$ and $\mathcal{A}\left(\mathcal{Z}_{R}\right)$ on $\widehat{\operatorname{CFAA}}\left(\beta^{\beta} \mathscr{H}^{\alpha}\right)$ as follows. Let $s$ be a subset of the $\beta$-arcs and $t$ a subset of the $\alpha$-arcs. Then define

$$
I(s) \cdot \mathbf{x} \cdot I(t)= \begin{cases}\mathbf{x} & \text { if } s \text { (resp. } t) \text { are the } \beta \text {-arcs (resp. } \alpha \text {-arcs) occupied by } \mathbf{x}, \\ 0 & \text { otherwise. }\end{cases}
$$

This action extends to actions of the rest of $\mathcal{A}\left(Z_{L}\right)$ and $\mathcal{A}\left(Z_{R}\right)$ by counting holomorphic curves in the usual way.

The type $D D$ and $D A$ modules associated to $\beta \mathscr{H}^{\alpha}$ are defined similarly.

For bimodules as for modules, there are two different geometric versions of algebraic duality.

Definition 3.23. Given a bordered Heegaard diagram with two boundary components $\mathscr{H}=(\Sigma, \boldsymbol{\alpha}, \boldsymbol{\beta}, \mathbf{z})$, let $-\mathscr{H}$ denote the bordered Heegaard diagram obtained from $\mathscr{H}$ by reversing the orientation on $\Sigma$, and $\overline{\mathscr{H}}$ the bordered Heegaard diagram obtained from $\mathscr{H}$ by calling the old $\alpha$-curves the new $\beta$ curves, and the old $\beta$-curves the new $\alpha$-curves.

(Compare Definition 3.11.) Lemma 3.12 has obvious analogues for bordered Heegaard diagrams with two boundary components:

Lemma 3.24. Let $\left(Y, \varphi_{L}: F\left(\mathcal{Z}_{L}^{\alpha}\right) \rightarrow \partial_{L} Y, \varphi_{R}: F\left(\mathcal{Z}_{R}^{\alpha}\right) \rightarrow \partial_{R} Y\right)$ be a strongly bordered 3-manifold with two boundary components. Let ${ }^{\alpha} \mathscr{H}^{\alpha}$ be an $\alpha$ - $\alpha$-bordered Heegaard diagram for $\left(Y, \varphi_{L}, \varphi_{R}\right)$. Then $\beta \mathscr{H}^{\beta}$ is a $\beta$-bordered Heegaard diagram for $\left(-Y, \varphi_{L} \circ K_{\beta, \alpha}, \varphi_{R} \circ K_{\beta, \alpha}\right)$. Similar statements hold in the cases that $Y$ is $\alpha-\beta$ bordered or $\beta$ - $\beta$-bordered.

Proof. This follows similarly to Lemma 3.12.

Proposition 3.25. If $\mathscr{H}$ is a bordered Heegaard diagram with two boundary components then we have the following dualities:

$$
\begin{aligned}
& \widehat{\operatorname{CFDD}}(\overline{\mathscr{H}},-\mathfrak{s}) \stackrel{\text { dual }}{\longleftrightarrow} \widehat{\operatorname{CFDD}}(\mathscr{H}, \mathfrak{s}), \\
& \widehat{\operatorname{CFAA}}(\overline{\mathscr{H}},-\mathfrak{s}) \stackrel{\stackrel{\text { dual }}{\longleftrightarrow} \widehat{\operatorname{CFAA}}(\mathscr{H}, \mathfrak{s}),}{\widehat{\operatorname{CFAD}}(\overline{\mathscr{H}},-\mathfrak{s}) \stackrel{\text { dual }}{\longleftrightarrow} \widehat{\operatorname{CFDA}}(\mathcal{H}, \mathfrak{s}) .}
\end{aligned}
$$

Proposition 3.26. If $\mathcal{H}$ is a bordered Heegaard diagram with two boundary components then we have the following dualities:

$$
\begin{aligned}
& \widehat{\mathrm{CFDD}}(-\mathcal{H},-\overline{\mathfrak{s}}) \stackrel{\text { dual }}{\longleftrightarrow} \widehat{\operatorname{CFDD}}(\mathscr{H}, \mathfrak{s}), \\
& \widehat{\mathrm{CFAA}}(-\mathcal{H},-\overline{\mathfrak{s}}) \stackrel{\stackrel{\text { dual }}{\longleftrightarrow} \widehat{\mathrm{CFAA}}(\mathscr{H}, \mathfrak{s}),}{\widehat{\mathrm{CFAD}}(-\mathcal{H},-\overline{\mathfrak{s}}) \stackrel{\text { dual }}{\longleftrightarrow} \widehat{\operatorname{CFDA}}(\mathscr{H}, \mathfrak{s}) .}
\end{aligned}
$$


(See Definition 2.12 for the definitions of the duals of various kinds of bimodules.)

Proof of Propositions 3.25 and 3.26. These propositions follow from similar arguments to Proposition 3.14. See also Proposition 3.15.

\section{An interpolating piece}

Fix a pointed matched circle $Z$. Our discussion of orientation reversal and proof of the Hom pairing theorem will rely on a particular arced $\alpha$ - $\beta$-bordered Heegaard diagram associated to $Z$, first introduced by Auroux [1]. The diagram, which we will denote $A Z(Z)$, is constructed as illustrated in Figure 4 and described below.

Let $k$ denote the genus of $F(Z)$. Let $T$ denote the triangle in $\mathbb{R}^{2}$ bounded by the $x$-axis, the $y$-axis, and the line $y+x=4 k+1$. Let $e_{y}$ (respectively $e_{x}$ ) denote the edge of $T$ along the $y$-axis (respectively $x$-axis) and $e_{D}$ the edge of $T$ along the line $x+y=4 k+1$. Let $\Sigma^{\prime}$ denote the quotient space of $T$ in which one identifies a small neighborhoods in $e_{D}$ of the points $(i, 4 k+1-i)$ and $(j, 4 k+1-j)$ if $i$ and $j$ are matched in $\mathcal{Z}$, in such a way that $\Sigma^{\prime}$ is an orientable surface of genus $k$ with one boundary component.

If $i$ and $j$ are matched in $\mathcal{Z}$ then the two vertical line segments $T \cap\{x=i\}$ and $T \cap\{x=j\}$ descend to give a single arc in $\Sigma^{\prime}$. Similarly, if $i$ and $j$ are matched then the horizontal line segments $T \cap\{y=4 k+1-i\}$ and $T \cap\{y=4 k+1-j\}$ descend to give a single arc in $\Sigma^{\prime}$. Let

$$
\boldsymbol{\beta}=\bigcup_{i=1}^{4 k}\{x=-i\} \subset \Sigma^{\prime}, \quad \boldsymbol{\alpha}=\bigcup_{i=1}^{4 k}\{y=i-4 k-1\} \subset \Sigma^{\prime}
$$

Finally, attach a 1 -handle to $\partial \Sigma^{\prime}$ between the points $(0,0)$ and $(4 k+1,0)$. Call the result $\Sigma$. Let $\mathbf{z}$ denote a neighborhood of the core of this 1 -handle. So, $\mathbf{z}$ is a rectangle in $\Sigma$ connecting the two boundary components.

Let $\mathrm{AZ}(\boldsymbol{Z})$ denote the diagram $(\Sigma, \boldsymbol{\alpha}, \boldsymbol{\beta}, \mathbf{z})$. We let $\partial_{R} \mathrm{AZ}(\boldsymbol{Z})$ denote the boundary component of $\mathrm{AZ}(\mathcal{Z})$ which intersects the $\beta$-arcs (so $\mathrm{AZ}(\mathcal{Z})$ is a $(\alpha, \beta)$-bordered Heegaard diagram). Note that $\partial_{L} A Z(Z)$ and $\partial_{R} A Z(Z)$ are twin pointed matched circles (in the sense of Definition 3.4). Sometimes, we denote this diagram by ${ }^{\alpha} A Z(Z){ }^{\beta}$ to call attention to the fact that it is $(\alpha, \beta)$-bordered, to distinguish it from ${ }^{\beta} \mathrm{AZ}(\mathcal{Z})^{\alpha}$, which is the same diagram but with the roles of $\partial_{L}$ and $\partial_{R}$ reversed. In particular, just as for ${ }^{\alpha} \mathrm{AZ}(\mathcal{Z})^{\beta}, \partial\left({ }^{\beta} \mathrm{AZ}(\mathcal{Z})^{\alpha}\right)=\mathcal{Z}^{\beta} \amalg \mathcal{Z}^{\alpha}$; and the bimodules $\widehat{\operatorname{CFAA}}\left({ }^{\alpha} \mathrm{AZ}(\mathcal{Z})^{\beta}\right)$ and $\widehat{\mathrm{CFAA}}\left({ }^{\beta} \mathrm{AZ}(\mathcal{Z})^{\alpha}\right)$ are canonically isomorphic. 

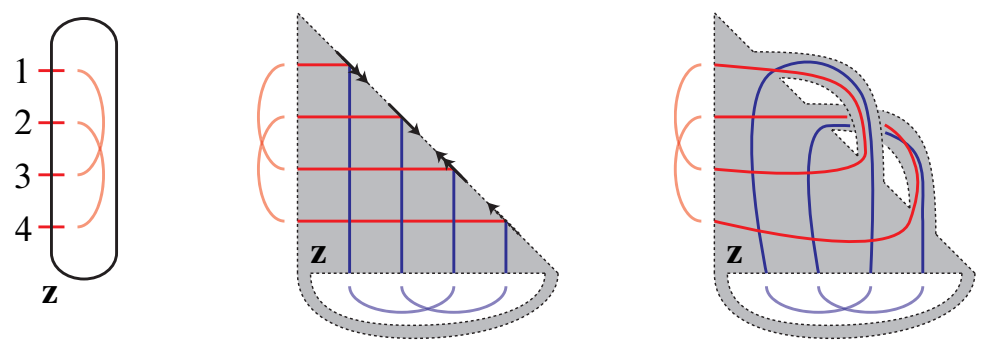

Figure 4. An example of $\mathbf{A Z}(\mathscr{Z})$. The example shown is for the genus 1 pointed matched circle. Left: the pointed matched circle $\mathcal{Z}$. Center and Right: two different depictions of $A Z(Z)$.

The following was proved by Auroux [1]. We recall the proof briefly here.

Proposition 4.1. The type $A A$ module $\widehat{\mathrm{CFAA}}(\mathrm{AZ}(\mathcal{Z}))$ associated to the diagram $A Z(Z)$, viewed as a left-right $\mathcal{A}(\mathcal{Z})-\mathcal{A}(Z)$-bimodule, is isomorphic to the bimodule $\mathcal{A}(\mathcal{Z})$.

Proof sketch. First, observe that the generators $\mathfrak{S}(\mathrm{AZ}(\mathbb{Z}))$ are in one-to-one correspondence with the standard basis for $\mathcal{A}(\mathcal{Z})$ by strand diagrams. Indeed, numbering the $\alpha$-circles from the bottom and the $\beta$-circles from the left, notice that the number of points in $\alpha_{i} \cap \beta_{j}$ is 2 if $i=j$, and otherwise the number of points is exactly the number of Reeb chords in $\mathbb{Z}$ starting at an endpoint of $\alpha_{i}$ and ending at an endpoint of $\alpha_{j}$. These intersections correspond to individual strands in a strand diagram: the intersection of $\alpha_{i} \cap \beta_{i}$ on the diagonal $e_{D}$ corresponds to a smeared horizontal strand, and other intersections correspond to Reeb chords or upward-sloping strands. An arbitrary generator of $\widehat{\mathrm{CFAA}}(\mathrm{AZ}(\mathbb{Z}))$ is a set of such intersection points, which thus correspond naturally to a strand diagram in the standard basis for $\mathcal{A}(\mathbb{Z})$.

To compute the $\mathcal{A}_{\infty}$-bimodule structure, one observes that $A Z(Z)$ is nice [20], so the differential on $\widehat{\mathrm{CFAA}}(\mathrm{AZ}(\mathcal{Z}))$ comes entirely from counting rectangles (there are no interior bigons), the only multiplications are $m_{2}$ 's, and these multiplications count half-strips. With this explicit description, it is straightforward to identify the differential and algebra actions on $\widehat{\mathrm{CFAA}}(\mathrm{AZ}(\mathcal{Z}))$ with those on $\mathcal{A}(\mathcal{Z})$. We refer the reader to [1] for more details.

Note that, since $\mathrm{AZ}(\mathcal{Z})$ has no closed $\alpha$ - or $\beta$-circles, $Y(\mathrm{AZ}(\mathcal{Z}))$ as an unparametrized 3-manifold is $F(Z) \times[0,1]$. Consequently, $A Z(Z)$ determines a homeomorphism from $F(\mathcal{Z})$ to itself preserving the preferred disk $D_{\alpha} \cup s \cup D_{\beta}$, well-defined up to isotopy fixing $D_{\alpha} \cup s \cup D_{\beta}$. We understand this map as follows.

Proposition 4.2. The diagram $\mathrm{AZ}(\mathcal{Z})$ represents a positive half Dehn twist, in the following sense. Let $\varphi_{\alpha} \mathrm{AZ}^{\beta}:-F\left(\mathcal{Z}^{\alpha}\right) \rightarrow F\left(Z^{\beta}\right)$ denote the homeomorphism associated (as in Construction 3.21) to the diagram ${ }^{\alpha} \mathrm{AZ}(\mathcal{Z})^{\beta}$, and $K_{\beta, \alpha}: F\left(Z^{\beta}\right) \rightarrow$ 
$-F\left(Z^{\alpha}\right)$ denote the canonical homeomorphism of twins (as in Definition 3.4). Then $K_{\beta, \alpha} \circ \varphi_{\alpha} Z^{\beta}=\tau_{\partial}^{1 / 2}:-F\left(Z^{\alpha}\right) \rightarrow-F\left(Z^{\alpha}\right)$. Likewise, $\varphi_{\alpha} \mathrm{AZ}^{\beta} \circ K_{\beta, \alpha}: F\left(\mathcal{Z}^{\beta}\right) \rightarrow$ $F\left(\mathcal{Z}^{\beta}\right), K_{\alpha, \beta} \circ \varphi_{\beta_{\mathrm{A}} Z^{\alpha}}:-F\left(Z^{\beta}\right) \rightarrow-F\left(\mathcal{Z}^{\beta}\right)$, and $\varphi_{\beta_{A} Z^{\alpha}} \circ K_{\alpha, \beta}: F\left(\mathcal{Z}^{\alpha}\right) \rightarrow F\left(Z^{\alpha}\right)$ all represent positive half Dehn twists on the respective surfaces.

Proof. We concentrate on the first case, $K_{\beta, \alpha} \circ \varphi_{\alpha} \mathrm{Z}^{\beta}$. Let $Y$ be the result of applying Construction 3.19 to the diagram $\operatorname{AZ}(Z)=(\bar{\Sigma}, \boldsymbol{\alpha}, \boldsymbol{\beta}, \mathbf{z})$. Since there are no closed $\alpha$ - or $\beta$-circles, $Y$ is given by

$$
[0,1] \times\left(D_{\alpha} \cup_{\partial_{L} \bar{\Sigma}} \cup \bar{\Sigma} \cup_{\partial_{R} \bar{\Sigma}} D_{\beta}\right) .
$$

With notation as in Construction 3.21,

(1) the map $\varphi_{L}: F\left(\mathcal{Z}^{\alpha}\right) \rightarrow\{0\} \times\left(D_{\alpha} \cup \bar{\Sigma} \cup D_{\beta}\right)$ sends $D_{\alpha}$ to $D_{\alpha} ; D_{\beta}$ to $D_{\beta}$; and $F\left(\mathcal{Z}^{\alpha}\right)$ to $\Sigma$, sending the cores of the 1-handles in $F\left(\mathcal{Z}^{\alpha}\right)$ to the $\alpha$-arcs;

(2) the map $\varphi_{R}: F\left(\mathcal{Z}^{\beta}\right) \rightarrow\{1\} \times\left(D_{\alpha} \cup \bar{\Sigma} \cup D_{\beta}\right)$ sends $D_{\alpha}$ to $D_{\alpha} ; D_{\beta}$ to $D_{\beta}$; and $F^{\circ}\left(\mathcal{Z}^{\beta}\right)$ to $\Sigma$, sending the cores of the 1-handles in $F^{\circ}\left(\mathcal{Z}^{\beta}\right)$ to the $\beta$-arcs;

(3) the map $\Phi:[0,1] \times F\left(\mathcal{Z}^{\beta}\right) \rightarrow[0,1] \times\left(D_{\alpha} \cup \bar{\Sigma} \cup D_{\beta}\right)$ is given by $\Phi(t, x)=$ $\left(t, \varphi_{R}(x)\right)$.

See Figure 5. In particular, by (3), the map $\varphi_{\mathrm{AZ}}$ is given by $\varphi_{R}^{-1} \circ\left(-\varphi_{L}\right):-F\left(\mathcal{Z}^{\alpha}\right) \rightarrow$ $F\left(\mathcal{Z}^{\beta}\right)$.

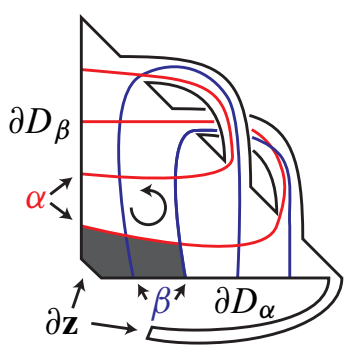

Figure 5. Identification of the homeomorphism associated to $A Z(Z)$. The diagram $A Z(Z) \backslash Z$ for $Z$ the pointed matched circle of genus 1 is shown. The small triangle identifying the homeomorphism as a positive half boundary Dehn twist is shaded.

So, we have a commutative diagram:

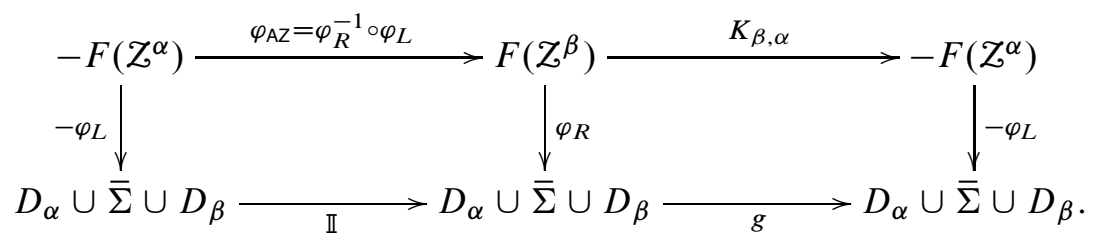



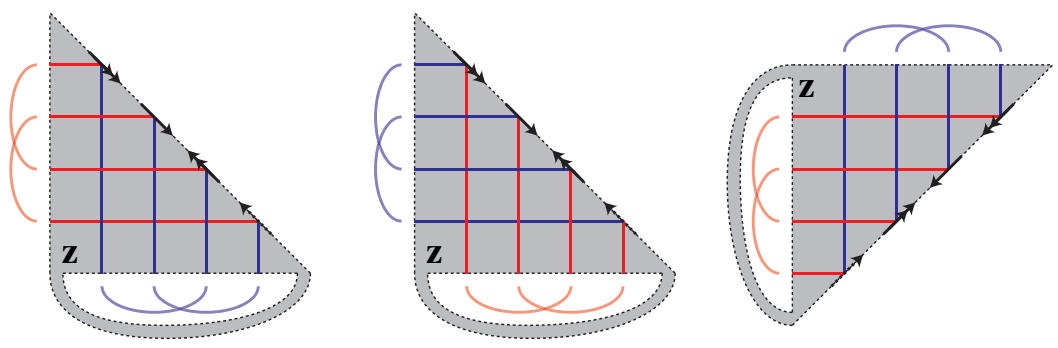

Figure 6. The diagram $\overline{\mathbf{A Z}}(\mathscr{Z})$. Left: the diagram $A Z(Z)$, for the genus 1 pointed matched circle. Center: the result of exchanging the $\alpha$ - and $\beta$-curves in $\operatorname{AZ}(\mathcal{Z})$. Right: the diagram $\overline{\mathrm{AZ}}(\mathrm{Z})$.

The map $K_{\beta, \alpha}$ takes the cores of the 1-handles in $F\left(Z^{\beta}\right)$ to the cores of the 1-handles in $F\left(\mathcal{Z}^{\alpha}\right)$. So, $-\varphi_{L} \circ K_{\beta, \alpha} \circ\left(\varphi_{R}\right)^{-1}=g$ is the map from $D_{\alpha} \cup \bar{\Sigma} \cup D_{\beta}$ to itself exchanging $D_{\alpha}$ and $D_{\beta}$ and taking each $\beta$-arc to the corresponding $\alpha$-arc. Hence, by Lemma 3.7, the map $g$ is a positive half boundary Dehn twist of $D_{\alpha} \cup \bar{\Sigma} \cup D_{\beta}$, and so $K_{\beta, \alpha} \circ \varphi_{\mathrm{AZ}}$ is a positive half boundary Dehn twist of $-F\left(Z^{\alpha}\right)$.

The other cases can be proved by similar commutative diagrams, or alternatively follow from the first case, using the observations that, on the one hand, $K_{\alpha, \beta} \circ \tau_{\partial}^{1 / 2} \circ$ $K_{\beta, \alpha}=\tau_{\partial}^{1 / 2}$ and, on the other hand, switching the left and right sides of $M_{\varphi}$ yields $M_{-\varphi^{-1}}$ and $\left(-\tau_{\partial}^{1 / 2}\right)=\tau_{\partial}^{1 / 2}$. (In both cases, the surface on which we apply $\tau_{\partial}^{1 / 2}$ changes.)

There is another interpolating piece that will be important, a kind of mirror image of $A Z(Z)$. More precisely, we can consider the diagram $\overline{A Z}(\mathcal{Z})$ obtained from $\operatorname{AZ}(\mathcal{Z})$ by switching the $\alpha$ - and $\beta$-curves and rotating the diagram clockwise 90 degrees. The boundary components of $\overline{\mathrm{AZ}}(\mathcal{Z})$ are naturally identified with $Z^{\alpha}$ and $Z^{\beta}$. Alternatively, $\overline{\mathrm{AZ}}(\mathcal{Z})$ is $-\mathrm{AZ}(-\mathcal{Z})$, obtained from $A Z(-Z)$ by reversing the orientation on the diagram (e.g., by reflecting across the $x$-axis). See Figure 6. By default, as with $A Z$, we view $Z^{\alpha}$ as the left boundary of $\overline{A Z}$ and $Z^{\beta}$ as the right boundary of $\overline{A Z}$, but when we want to make this convention or the opposite one explicit we will write ${ }^{\alpha} \overline{\mathrm{AZ}}^{\beta}$ or ${ }^{\beta} \overline{\mathrm{AZ}}^{\alpha}$, respectively.

With no additional work, we get the following algebraic description of the bordered invariants for $\overline{\mathrm{AZ}}(\mathcal{Z})$ :

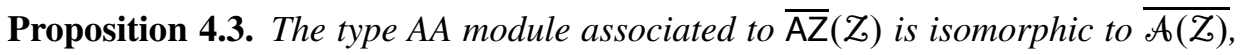
the dual module to $\mathcal{A}(\mathcal{Z})$.

Proof. This follows from Propositions 4.1 and 3.25.

Proposition 4.4. Let $\varphi_{\alpha \overline{\mathrm{AZ}}^{\beta}}:-F\left(\mathcal{Z}^{\alpha}\right) \rightarrow F\left(\mathcal{Z}^{\beta}\right)$ denote the homeomorphism associated (as in Construction 3.21) to the diagram ${ }^{\alpha} \overline{\mathrm{AZ}}^{\beta}$, and $K_{\beta, \alpha}: F\left(\mathcal{Z}^{\beta}\right) \rightarrow-F\left(\mathcal{Z}^{\alpha}\right)$ 
denote the canonical homeomorphism of twins (as in Definition 3.4). Then $K_{\beta, \alpha} \circ$ $\varphi_{\alpha} \overline{\mathrm{AZ}}^{\beta}=\tau_{\partial}^{-1 / 2}:-F\left(\mathcal{Z}^{\alpha}\right) \rightarrow-F\left(Z^{\alpha}\right)$. Likewise, $\varphi_{\alpha} \overline{\mathrm{AZ}}^{\beta} \circ K_{\beta, \alpha}: F\left(Z^{\beta}\right) \rightarrow F\left(\mathcal{Z}^{\beta}\right)$, $K_{\alpha, \beta} \circ \varphi_{\beta} \overline{\mathrm{AZ}}^{\alpha}:-F\left(\mathbb{Z}^{\beta}\right) \rightarrow-F\left(Z^{\beta}\right)$, and $\varphi_{\beta} \overline{\mathrm{AZ}}^{\alpha} \circ K_{\alpha, \beta}: F\left(\mathbb{Z}^{\alpha}\right) \rightarrow F\left(Z^{\alpha}\right)$ all represent negative half Dehn twists on the respective surfaces.

Proof. This follows along the lines of Proposition 4.2: combine the construction of the associated homeomorphism with Lemma 3.7. Alternatively, note that exchanging the $\alpha$-and $\beta$-circles has the effect of reversing the orientation on the three-manifold. This, in turn, exchanges positive and negative Dehn twists, so the result follows from Proposition 4.2.

In Section 5, the following corollary of Propositions 4.2 and 4.4 will be useful, particularly as sanity checks on the signs / presence of boundary Dehn twists.

Corollary 4.5. The diagram ${ }^{\alpha} \mathrm{AZ}(-\mathcal{Z})^{\beta} \cup^{\beta} \mathrm{AZ}(\mathcal{Z})^{\alpha}$ represents $\tau_{\partial}: F\left(\mathcal{Z}^{\alpha}\right) \rightarrow F\left(Z^{\alpha}\right)$. The diagram ${ }^{\alpha} \mathrm{AZ}(-\mathbb{Z})^{\beta} \cup{ }^{\beta} \overline{\mathrm{AZ}}(\mathbb{Z})^{\alpha}$ represents the identity map of $F\left(\mathcal{Z}^{\alpha}\right)$.

See Figure 7. More identities of this kind are given at the end of Appendix A.

Proof. For the first statement, we have

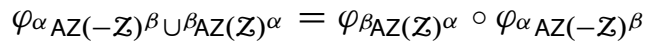

$$
\begin{aligned}
& \left.=\left(\varphi_{\left.\beta_{\mathrm{AZ}(Z)}\right)^{\alpha}} \circ K_{\alpha, \beta}\right) \circ\left(K_{\beta, \alpha} \circ \varphi_{\alpha} \mathrm{AZ}(-Z)\right)^{\beta}\right) \\
& =\left(\tau_{\partial}^{1 / 2}: F\left(Z^{\alpha}\right) \longrightarrow F(\mathcal{Z})^{\alpha}\right) \circ\left(\tau_{\partial}^{1 / 2}: F\left(Z^{\alpha}\right) \longrightarrow F\left(Z^{\alpha}\right)\right) \\
& =\tau_{\partial}: F\left(Z^{\alpha}\right) \longrightarrow F\left(Z^{\alpha}\right) \text {. }
\end{aligned}
$$

The second statement is similar, except the two half boundary twists go in opposite directions and so cancel.
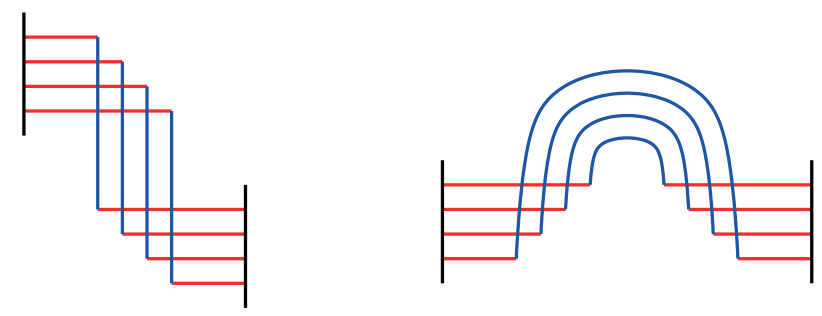

Figure 7. Gluing $\mathbf{A Z}(\mathscr{Z})$ to itself and its inverse. The left picture represents the Heegaard diagram ${ }^{\alpha} \mathrm{AZ}(-\mathcal{Z})^{\beta} \cup{ }^{\beta} \mathrm{AZ}(\mathcal{Z})^{\alpha}$ (which in turn represents $\tau_{\partial}^{-1}$ ), while the right illustrates ${ }^{\alpha} \mathrm{AZ}(-\mathbb{Z})^{\beta} \cup{ }^{\beta} \overline{\mathrm{AZ}}(\mathbb{Z})^{\alpha}$ (which represents the identity). 
Lemma 4.6. Let ${ }^{\alpha} \mathscr{H}^{\alpha}$ be an $\alpha$ - $\alpha$-bordered Heegaard diagram, with $\partial_{L} \mathscr{H}=Z_{L}$ and $\partial_{R} \mathscr{H}=Z_{R}$. Then the two $(\alpha, \beta)$-bordered Heegaard diagrams

$$
{ }^{\alpha} \mathscr{H}^{\alpha}{ }_{\partial_{R}} \cup_{\partial_{L}}{ }^{\alpha} \mathrm{AZ}\left(-\mathcal{Z}_{R}\right)^{\beta} \text { and }{ }^{\alpha} \mathrm{AZ}\left(\mathcal{Z}_{L}\right)^{\beta}{ }_{\partial_{R}} \cup_{\partial_{L}}{ }^{\beta}(-\overline{\mathcal{H}})^{\beta}
$$

represent the same strongly bordered three-manifold.

The intuition here is that the negative half Dehn twist represented by AZ can be "pulled through" from one side of $Y(\mathscr{H})$ to the other, but we have to turn over the Morse function on $Y(\mathscr{H})$ in the process. (Note that $Y(-\overline{\mathscr{H}})$ is orientation-preserving homeomorphic to $Y(\mathscr{H})$.)

Proof. Let $Y$ be a three-manifold with two boundary components. Then $Y$ can be factored as a product of elementary cobordisms, each of which corresponds to attaching a one-handle or two-handle to $\partial_{L} Y$. Moreover, a strongly bordered threemanifold can be factored into the following simple pieces:

- mapping cylinders for homeomorphisms, and

- elementary cobordisms from $\mathbb{Z} \# Z_{1}$ to $\mathbb{Z}$, or from $\mathbb{Z}$ to $\mathbb{Z} \# Z_{1}$, where $\mathbb{Z}_{1}$ denotes the genus 1 pointed matched circle, obtained by attaching a two-handle along the $\infty$-framed curve in $F^{\circ}\left(Z_{1}\right) \subset F\left(Z_{\left.\# Z_{1}\right)}\right.$ (as in Figure 8).

In particular, any Heegaard diagram $\mathscr{H}_{0}$ is equivalent to a diagram $\mathscr{H}$ which can be written as a juxtaposition of pieces of these two forms, and obviously $-\overline{\mathscr{H}_{0}}$ is then equivalent to $-\overline{\mathscr{H}}$. Thus, it suffices to check the result for these two kinds of elementary pieces.

For the first simple piece, let $\mathscr{H}_{\varphi}$ be the standard Heegaard diagram for a mapping class $\varphi: F\left(Z_{1}\right) \rightarrow F\left(Z_{2}\right)$, and let $\mathrm{AZ}\left(\mathcal{Z}_{1}\right)(\boldsymbol{\alpha}, \varphi(\boldsymbol{\beta}))$ (for instance) be the diagram defined like ${ }^{\alpha} \mathrm{A} Z\left(Z_{1}\right)^{\beta}$ but using the image of the $\beta$-curves on $Z_{2}$ under the mapping class $\varphi$, thought of as acting on the complement of $\mathbf{z}$ in the Heegaard surface. (Thus $A Z\left(Z_{1}\right)(\boldsymbol{\alpha}, \boldsymbol{\beta})={ }^{\alpha} A Z\left(Z_{1}\right)^{\beta}$.) Then we have equivalences of Heegaard diagrams

$$
\begin{aligned}
\mathscr{H}_{\varphi} \cup \mathrm{AZ}\left(-\mathrm{Z}_{2}\right)(\boldsymbol{\alpha}, \boldsymbol{\beta}) & \simeq \mathrm{AZ}\left(-Z_{2}\right)(\varphi(\boldsymbol{\alpha}), \boldsymbol{\beta}) \\
& \simeq \mathrm{AZ}\left(-Z_{1}\right)\left(\boldsymbol{\alpha}, \varphi^{-1}(\boldsymbol{\beta})\right) \\
& \simeq \mathrm{AZ}\left(-\mathrm{Z}_{1}\right)(\boldsymbol{\alpha}, \boldsymbol{\beta}) \cup\left(-\overline{\mathscr{H}_{\varphi}}\right),
\end{aligned}
$$

where we are using the fact that gluing mapping cylinders corresponds to twisting the parametrization [10], Lemma 5.30, a homeomorphism of diagrams, and the gluing property again.

For the second simple piece (elementary cobordisms of the specified form), the needed sequence of handleslides and destabilizations is easy to find; see Figure 8, where we have illustrated the case where $Z$ is empty. 

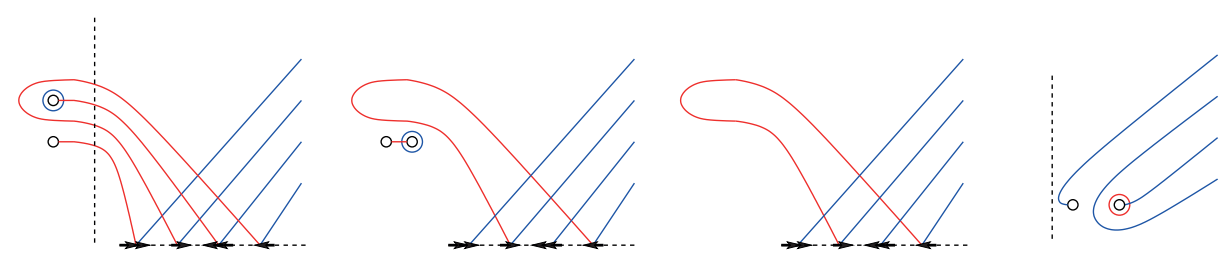

Figure 8. Commuting $\mathbf{A Z}$ past an elementary cobordism. Heegaard moves exhibiting the identification ${ }^{\alpha} \mathscr{H}^{\alpha} \cup \mathrm{AZ}\left(-\mathcal{Z}_{R}\right) \simeq \mathrm{AZ}\left(\mathcal{Z}_{R}\right) \cup{ }^{\beta}(-\overline{\mathscr{H}})^{\beta}$, in the case where $\mathscr{H}$ represents an elementary cobordism from the two-sphere to a genus one surface (so $A Z\left(Z_{L}\right)$ is empty). The first diagram is ${ }^{\alpha} \mathscr{H}^{\alpha} \cup A Z\left(-Z_{R}\right)$, the second is gotten by a sequence of handleslides, the third by a destabilization, and the fourth by an isotopy (and a homeomorphism of diagrams).

\section{Consequences}

5.1. Orientation reversal and the Hom pairing theorem for modules. As a warmup for the proofs of our main theorems, we start with the module case, in which the notation is a little simpler to follow, and the Dehn twists disappear.

Proof of Theorem 2. Fix an $\alpha$-bordered Heegaard diagram $\mathscr{H}^{\alpha}$ for $(Y, \varphi: F(Z) \rightarrow$ $Y$ ). By Lemma 4.6 in the case when one boundary is empty (which is essentially the fact that boundary Dehn twists have no effect on 3-manifolds with just one boundary component), the $\alpha$-bordered Heegaard diagram $\overline{\mathscr{H}}^{\beta}{ }_{\partial} \cup_{\partial_{R}} \mathrm{AZ}(-\mathcal{Z})$ represents $(-Y, \varphi$ : $F(-\mathbb{Z}) \rightarrow-Y)$. We have now that

$$
\begin{aligned}
\widehat{\mathrm{CFA}}(-Y) & \cong \widehat{\mathrm{CFA}}\left(\overline{\mathscr{H}}^{\beta}{ }_{\partial} \cup_{\partial_{R}} \mathrm{AZ}(-\mathcal{Z})\right) \\
& \simeq \widehat{\operatorname{CFD}}\left(\overline{\mathcal{H}}^{\beta}\right) \otimes \widehat{\operatorname{CFAA}}(\mathrm{AZ}(-\mathcal{Z})) \\
& \cong \widehat{\operatorname{CFD}}\left(\overline{\mathscr{H}}^{\beta}\right) \otimes \mathcal{A}(-\mathcal{Z}) \\
& \cong \widehat{\widehat{C F D}\left(\mathscr{H}^{\alpha}\right)} \otimes \mathcal{A}(-\mathcal{Z}) \\
& \simeq \operatorname{Mor}_{\mathcal{A}(-\mathcal{Z})}(\widehat{\operatorname{CFD}}(Y), \mathcal{A}(-\mathcal{Z}))
\end{aligned}
$$

Here, the second line uses the pairing theorem (in a form using bimodules; see [10], Theorem 11), the third uses Proposition 4.1, the fourth uses Proposition 3.14, and the last uses Proposition 2.7. This proves (1.1).

Eq. (1.2) follows from (1.1) and Theorem 2.3. That is,

$$
\begin{aligned}
\widehat{\mathrm{CFA}}(-Y) & \simeq \operatorname{Mor}^{\mathcal{A}(-Z)}\left(\widehat{\mathrm{CFD}}(Y), \widehat{\mathcal{A}(-Z)}[\mathbb{I}]_{\mathcal{A}(-Z)}\right) \\
& \simeq \operatorname{Mor}_{\mathcal{A}(\mathcal{Z})}(\widehat{\mathrm{CFA}}(Y), \widehat{\mathrm{CFAA}}(\mathbb{I})),
\end{aligned}
$$

where the first equivalence is (1.1) and the second follows by tensoring both domain and range with $\widehat{\mathrm{CFAA}}(\mathbb{I})$, which is an equivalence of categories (Theorem 2.3). Tensoring both sides with $\widehat{\mathrm{CFDD}}(\mathbb{I})$ gives (1.2). 
Remark 5.1. In the context of bordered sutured manifolds [22] with upper and lower pieces of the boundary $R^{+}$and $R^{-}$, the operation $\mathscr{H} \leadsto \overline{\mathcal{H}}_{\partial} \cup_{\partial_{R}} \mathrm{AZ}(\mathcal{Z})$ corresponds to a slightly more complicated operation than mere orientation reversal. When one switches the $\alpha$ - and $\beta$-curves on a diagram for a bordered sutured manifold, the roles of $R_{+}$and $R_{-}$are exchanged. Attaching $Y(\mathrm{AZ}(Z))$ then corresponds to introducing a half Dehn twist along the preferred disk in $F(\mathcal{Z})$, as in Proposition 4.2. (As above, Dehn twists around the preferred disk in $F(Z)$ disappear if one instead glues to a 3-manifold with one boundary component.) See Figure 9 for an example where this operation is non-trivial.
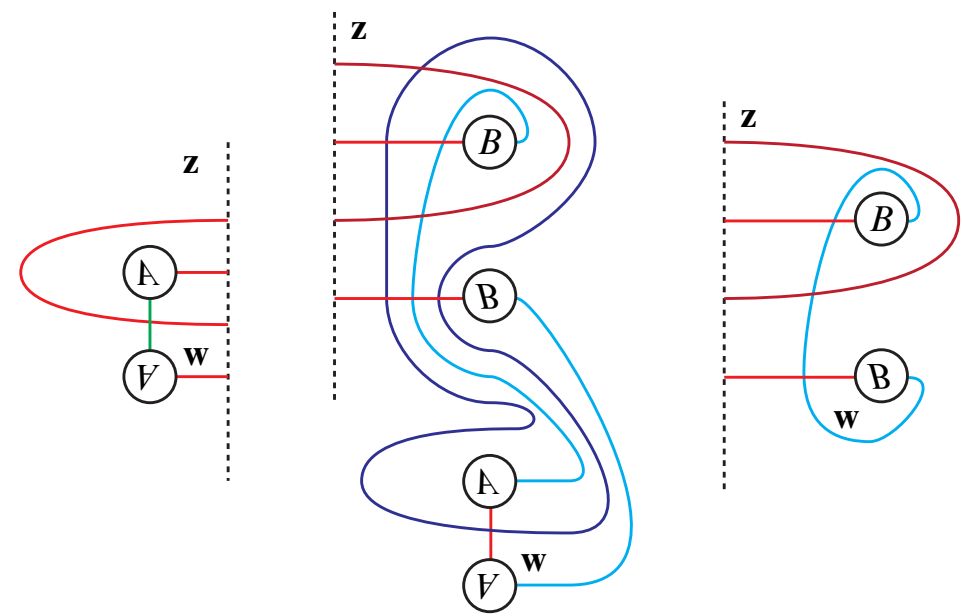

Figure 9. Gluing $\mathbf{A Z}\left(\mathscr{Z}_{\boldsymbol{C}}\right)$ to a doubly-pointed Heegaard diagram. Left: A doubly-pointed $\alpha$-bordered Heegaard diagram $\mathscr{H}$ for the core of a 0 -framed solid torus. Center: the result $\mathrm{AZ}(\mathcal{Z}) \cup_{\partial} \mathscr{H}^{\beta}$ of gluing the interpolating piece to $\mathscr{H}^{\beta}$. Right: a destabilization of $\mathrm{AZ}(\mathcal{Z}) \cup_{\partial}$ $\mathscr{H}^{\beta}$.

Proof of Theorem 1. Using Proposition 2.7, Theorem 2, and the usual version of the pairing theorem [9], Theorem 1.3, in turn gives:

$$
\begin{aligned}
\operatorname{Mor}_{\mathcal{A}(-Z)}\left(\widehat{\mathrm{CFD}}\left(Y_{1}\right), \widehat{\mathrm{CFD}}\left(Y_{2}\right)\right) & \cong \operatorname{Mor}_{\mathcal{A}(-Z)}\left(\widehat{\mathrm{CFD}}\left(Y_{1}\right), \mathbb{I}\right) \otimes \widehat{\mathrm{CFD}}\left(Y_{2}\right) \\
& \simeq \widehat{\mathrm{CFA}}\left(-Y_{1}\right) \otimes \widehat{\operatorname{CFD}}\left(Y_{2}\right) \\
& \simeq \widehat{\mathrm{CF}}\left(-Y_{1} \cup_{\partial} Y_{2}\right) .
\end{aligned}
$$

Taking homology gives the first isomorphism from Theorem 1. The second isomorphism then follows from Corollary 2.4. 


\subsection{Conjugation invariance}

Proposition 5.2. Suppose that ${ }^{\alpha} \mathscr{H}^{\alpha}$ is an $\alpha$ - $\alpha$-bordered Heegaard diagram with boundary components $Z_{1}$ and $Z_{2}$. By the isomorphisms $\mathcal{A}\left(-\left(-Z_{i}\right)\right)^{\mathrm{op}} \cong \mathcal{A}\left(Z_{i}\right)^{\mathrm{op}} \cong$ $\mathcal{A}\left(-Z_{i}\right)$, we can view both $\widehat{\operatorname{CFDD}}\left({ }^{\alpha} \mathscr{H}^{\alpha}\right)$ and $\widehat{\operatorname{CFDD}}\left(-{ }^{\beta} \overline{\mathscr{H}}^{\beta}\right)$ as left-left $\mathcal{A}\left(-Z_{1}\right) \otimes$ $\mathcal{A}\left(-Z_{2}\right)$-modules. Under this identification and similar ones for other modules, we have isomorphisms

$$
\begin{aligned}
& \widehat{\operatorname{CFDD}}(\mathscr{H}, \mathfrak{s}) \cong \widehat{\operatorname{CFDD}}(-\overline{\mathscr{H}}, \overline{\mathfrak{s}}), \\
& \widehat{\operatorname{CFAA}}(\mathscr{H}, \mathfrak{s}) \cong \widehat{\operatorname{CFAA}}(-\overline{\mathscr{H}}, \overline{\mathfrak{s}}), \\
& \widehat{\operatorname{CFDA}}(\mathscr{H}, \mathfrak{s}) \cong \widehat{\operatorname{CFDA}}(-\overline{\mathscr{H}}, \overline{\mathfrak{s}}) .
\end{aligned}
$$

Proof. The identification of the complexes $\widehat{\operatorname{CFDD}}(\mathscr{H})$ with $\widehat{\operatorname{CFDD}}(-\overline{\mathscr{H}})$ is supplied by combining Propositions 3.25 and 3.26. The conjugation on the $\operatorname{spin}^{c}$ structures comes from the observation that, when $\mathbf{x}$ is viewed as a generator for the bordered Floer homology of $\mathscr{H}$, its corresponding vector field points in the opposite direction from that of $\overline{\mathbf{x}}$ when viewed as a generator for the bordered Floer homology of $-\overline{\mathscr{H}}$.

Proof of Theorems 3 and 9. We will prove Theorem 9; Theorem 3 can be viewed as a special case where one of the boundary components is empty (or, if one prefers, $S^{2}$ ), after noting that $\tau_{\partial}$ acts trivially on bordered 3-manifolds with only one boundary component.

To establish (1.16), we must show that

$$
\begin{aligned}
& \left(\mathcal{A}\left(-Z_{1}\right)_{\mathcal{A}}\left(Z_{1}\right), \mathcal{A}\left(-Z_{1}\right) \otimes \mathcal{A}\left(-Z_{2}\right) \mathcal{A}\left(Z_{2}\right), \mathcal{A}\left(-Z_{2}\right)\right) \otimes^{\mathcal{A}\left(-Z_{1}\right), \mathcal{A}\left(-Z_{2}\right)} \widehat{\operatorname{CFDD}}(Y, \mathfrak{s}) \\
& \simeq \widehat{\mathrm{CFAA}}\left(\tau_{\partial}(Y), \overline{\mathfrak{s}}\right)_{\mathcal{A}}\left(Z_{1}\right), \mathcal{A}\left(Z_{2}\right) \text {, }
\end{aligned}
$$

where $\mathcal{A}\left(-Z_{i}\right)_{\mathcal{A}}\left(Z_{i}\right), \mathcal{A}\left(-Z_{i}\right)$ denotes the $\mathcal{A}\left(-Z_{i}\right)$-bimodule $\mathcal{A}\left(-Z_{i}\right)$, viewed as a module with two right actions.

Fix an $\alpha$ - $\alpha$-bordered Heegaard diagram ${ }^{\alpha} \mathscr{H}^{\alpha}$ for $Y$ (with $\partial_{L} \mathscr{H}=\mathcal{Z}_{1}$ and $\partial_{R} \mathscr{H}=$ $Z_{2}$ ), so that $\widehat{\mathrm{CFAA}}(Y, \mathfrak{s})$ is given by the bimodule $\widehat{\mathrm{CFAA}}(\mathcal{H}, \mathfrak{s})$ associated to $\mathscr{H}$ and $\mathfrak{s}$, and also (according to Proposition 5.2$)$ by the bimodule $\widehat{\mathrm{CFAA}}(-\overline{\mathscr{H}}, \overline{\mathfrak{s}})$ associated to the $\beta$ - $\beta$-bordered version ${ }^{\beta}(-\overline{\mathscr{H}})^{\beta}$ and the conjugate $\operatorname{spin}^{c}$-structure $\overline{\mathfrak{s}}$. Now, glue a copy of ${ }^{\alpha} \mathrm{AZ}\left(\mathcal{Z}_{1}\right)^{\beta}$ and a copy of ${ }^{\beta} \mathrm{AZ}\left(\mathcal{Z}_{2}\right)^{\alpha}$ to the $\partial_{L}$ and $\partial_{R}$ boundary components of $-\overline{\mathscr{H}}$ respectively.

Combining Proposition 4.1, the pairing theorem, and Proposition 5.2, we get

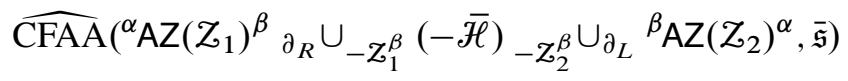

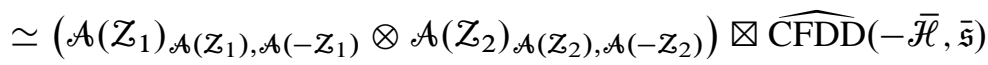

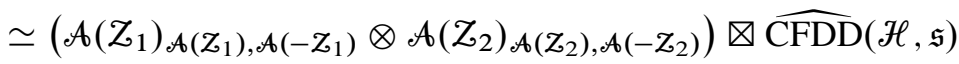

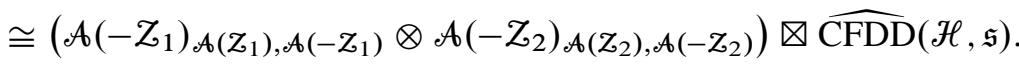


The last isomorphism comes from the tautological identification of bimodules $A_{A, A^{\text {op }}}$ $\cong\left(A^{\mathrm{op}}\right)_{A, A^{o p}}$, together with the usual identification of $\mathcal{A}(-\mathbb{Z}) \cong \mathcal{A}(\mathbb{Z})^{\mathrm{op}}$.

We can alternatively move $A Z\left(Z_{1}\right)$ past $-\overline{\mathscr{H}}$ as in Lemma 4.6. This gives

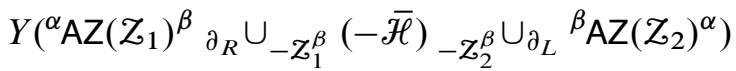

$$
\begin{aligned}
& \simeq Y\left(\mathscr{H}_{\partial_{R}} \cup_{\partial_{L}}{ }^{\alpha} \mathrm{AZ}\left(-\mathcal{Z}_{2}\right)^{\beta}{ }_{\partial_{R}} \cup_{\partial_{L}}{ }^{\beta} \mathrm{AZ}\left(\mathcal{Z}_{2}\right)^{\alpha}\right) \\
& \simeq \tau_{\partial}(Y(\mathscr{H})) \text {. }
\end{aligned}
$$

Here, the equivalence is of strongly bordered 3-manifolds, and the last line follows from Corollary 4.5. Eqs. (5.5) and (5.4) combine to establish (5.3), which is equivalent to $(1.16)$.

Eq. (1.17) is immediate from (1.16): replace $Y$ with $\tau_{\partial}^{-1}(Y), \mathfrak{s}$ with $\overline{\mathfrak{s}}$, and viewing the right actions as left actions.

Eq. (1.18) follows from the first two parts by tensoring (1.16) applied to $Y$ with (1.17) applied to $\mathbb{I}_{\mathcal{Z}_{2}}$ :

$$
\begin{aligned}
& \widehat{\operatorname{CFDD}}(Y, \mathfrak{s})_{\mathcal{A}}\left(Z_{1}\right), \mathcal{A}\left(Z_{2}\right) \widetilde{\otimes}_{\mathcal{A}}\left(Z_{2}\right), \mathcal{A}\left(-Z_{2}\right) \widehat{\operatorname{CFAA}}\left(\mathbb{I}_{Z_{2}}, \mathfrak{t}\right) \\
& \quad \simeq \widehat{\operatorname{CFAA}}\left(\tau_{\partial}(Y), \overline{\mathfrak{s}}\right)_{\mathcal{A}}\left(Z_{1}\right), \mathcal{A}\left(Z_{2}\right) \widetilde{\otimes}_{\mathcal{A}}\left(Z_{2}\right), \mathcal{A}\left(-Z_{2}\right) \widehat{\operatorname{CFDD}}\left(\tau_{\partial}^{-1}, \overline{\mathfrak{t}}\right),
\end{aligned}
$$

which reduces to the desired result.

Proof of Corollary 4. Eqs. (1.4) and (1.5) are immediate from Theorem 3 and the observations that

$$
\begin{aligned}
& \mathcal{A}(Z)_{\mathcal{A}(Z), \mathcal{A}(-Z)} \nabla^{\mathcal{A}(-Z)} \widehat{\mathrm{CFD}}(Y, \mathfrak{s})
\end{aligned}
$$

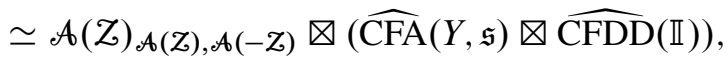

$$
\begin{aligned}
& \widehat{\mathrm{CFA}}(Y, \mathfrak{s})_{\mathcal{A}(\mathcal{L})} \\
& \simeq \widehat{\mathrm{CFAA}}(\mathbb{I})_{\mathcal{A}(Z), \mathcal{A}(-Z)} \otimes^{\mathcal{A}(-Z)} \widehat{\mathrm{CFD}}(Y, \mathfrak{s}) .
\end{aligned}
$$

(both of which follow from the pairing theorem).

\subsection{Orientation reversal and the Hom pairing theorems for bimodules}

Proof of Theorem 5. We start by proving (1.6). By [10], Corollary 2.3.37, the complex of type $D$ morphisms between two type $D$ structures is quasi-isomorphic to the complex of $\mathcal{A}_{\infty}$-morphisms between their modulifications, so (1.6) is equivalent to

$$
\operatorname{Mor}^{\mathcal{A}_{1}^{\prime}}\left(\mathcal{A}_{2}^{\prime} \otimes \mathcal{A}_{1}^{\prime}, \mathcal{A}_{2}^{\prime} \widehat{\operatorname{CFDD}}(Y),{ }^{\mathcal{A}_{1}^{\prime}}[\mathbb{I}]_{\mathcal{A}_{1}^{\prime}}\right) \simeq \widehat{\mathrm{CFAA}}(-Y)_{\mathcal{A}_{1}^{\prime}, \mathcal{A}_{2}^{\prime}} .
$$

Using the definition of Mor in terms of $\nabla$ (Proposition 2.14), we want to show that

$$
\overline{\mathcal{A}_{2}^{\prime} \otimes \mathcal{A}_{1}^{\prime}, \mathcal{A}_{2}^{\prime} \widehat{\mathrm{CFDD}}(Y)} \otimes \mathcal{A}_{1}^{\prime} \simeq \widehat{\mathrm{CFAA}}(-Y)_{\mathcal{A}_{1}^{\prime}, \mathcal{A}_{2}^{\prime}}
$$


Since taking duals respects $\otimes($ Lemma 2.15), this boils down to

$$
\overline{\mathcal{A}_{1}^{\prime}, \mathcal{A}_{2}^{\prime} \widehat{\mathrm{CFDD}}(Y)} \otimes \overline{\mathcal{A}_{2}^{\prime}} \otimes \mathcal{A}_{1}^{\prime} \simeq \widehat{\mathrm{CFAA}}(-Y)_{\mathcal{A}_{1}^{\prime}, \mathcal{A}_{2}^{\prime}} .
$$

Now, fix a Heegaard diagram ${ }^{\alpha} \mathscr{H}^{\alpha}$, with $\partial_{L} \mathscr{H}=\mathcal{Z}_{1}$ and $\partial_{R} \mathscr{H}=\mathcal{Z}_{2}$, for $\left(Y, \varphi_{L}: F\left(\mathcal{Z}_{1}\right) \rightarrow \partial_{L} Y, \varphi_{R}: F\left(\mathcal{Z}_{2}\right) \rightarrow \partial_{R} Y\right)$. According to Lemma $3.24,{ }^{\beta} \overline{\mathcal{H}}^{\beta}$ is a Heegaard diagram for $-Y$ with bordering $\varphi_{L} \circ K_{\beta, \alpha}$ and $\varphi_{R} \circ K_{\beta, \alpha}$. By Propositions 4.2 and 4.4 and Lemma 4.6, ${ }^{\alpha} \mathrm{AZ}\left(-\mathcal{Z}_{1}\right)^{\beta}{ }_{\partial_{R}} \cup_{F\left(\mathcal{Z}_{1}^{\beta}\right)}{ }^{\beta} \overline{\mathscr{H}}^{\beta}{ }_{F\left(\mathcal{Z}_{2}^{\beta}\right)} \cup_{\partial_{L}}{ }^{\beta} \overline{\mathrm{AZ}}\left(-\mathcal{Z}_{2}\right)^{\alpha}$ also represents $-Y$, with the bordering $-\varphi_{L}$ and $-\varphi_{R}$. By the pairing theorem and Propositions 3.25, 4.1 and 4.3,

$$
\begin{aligned}
& \widehat{\mathrm{CFAA}}\left({ }^{\alpha} \mathrm{AZ}\left(-\mathcal{Z}_{1}\right)^{\beta}{ }_{\partial_{R}} \cup_{F\left(\mathcal{Z}_{1}^{\beta}\right)}{ }^{\beta} \overline{\mathscr{H}}^{\beta}{ }_{F\left(Z_{2}^{\beta}\right)} \cup_{\partial_{L}} \beta \overline{\mathrm{AZ}}\left(-Z_{2}\right)^{\alpha}\right) \\
& \simeq \overline{\widehat{\operatorname{CFDD}}\left({ }^{\alpha} \mathscr{H}^{\alpha}\right)} \otimes \overline{\mathcal{A}_{2}^{\prime}} \otimes \mathcal{H}_{1}^{\prime} .
\end{aligned}
$$

This implies (5.7), and hence (5.6).

To prove (1.7), start by tensoring both sides of (5.6) with $\widehat{\operatorname{CFDD}}\left(\mathbb{I}_{Z_{1}}\right)$, to obtain

$$
\operatorname{Mor}^{\mathscr{A}_{1}^{\prime}}\left(\mathscr{A}_{2}^{\prime} \otimes \mathcal{A}_{1}^{\prime}, \mathcal{A}_{2}^{\prime} \widehat{\operatorname{CFDD}}(Y),{ }^{\mathcal{A}_{1}^{\prime}, \mathcal{A}_{1}} \widehat{\operatorname{CFDD}}\left(\mathbb{I}_{Z_{1}}\right)\right) \simeq{ }^{\mathcal{A}_{1}} \widehat{\operatorname{CFDA}}(-Y)_{\mathcal{A}_{2}^{\prime}} .
$$

Since tensoring over $\mathcal{A}_{1}^{\prime}$ with $\widehat{\mathrm{CFAA}}\left(\mathbb{I}_{Z_{1}}\right)$ gives an equivalence of categories which carries type $D$ bordered invariants to type $A$ bordered invariants (a bimodule analogue of Theorem 2.3; see [10], Lemma 9.1), this is the same as

$$
\operatorname{Mor}_{\mathcal{A}_{1}}\left(\mathcal{A}_{2}^{\prime} \otimes \mathcal{A}_{2}^{\prime} \widehat{\operatorname{CFDA}}(Y)_{\mathcal{A}_{1}},,^{\mathcal{A}_{1}}[\mathbb{I}]_{\mathcal{A}_{1}}\right) \simeq{ }^{\mathcal{A}_{1}} \widehat{\mathrm{CFDA}}(-Y)_{\mathscr{A}_{2}^{\prime}} .
$$

After lowering the index $\mathcal{A}_{1}$, this is exactly (1.7).

Eq. (1.8) is immediate from (1.7) and (1.18).

To prove (1.9), observe that, by viewing left actions as right actions by the opposite algebra, (1.6) is equivalent to

$$
\operatorname{Mor}_{\mathcal{A}_{1}}\left(\widehat{\operatorname{CFDD}}(Y)_{\mathcal{A}_{1}, \mathcal{A}_{2}}, \mathcal{A}_{1}\right) \simeq{\mathcal{A}_{1}, \mathcal{A}_{2}}_{2} \widehat{\operatorname{CFAA}}(-Y) .
$$

Applying Theorem 9 to both sides gives

$$
\operatorname{Mor}_{\mathcal{A}_{1}}\left(\widehat{\operatorname{CFAA}}\left(\tau_{\partial}(Y)\right)_{\mathcal{A}_{1}, \mathcal{A}_{2}}, \mathcal{A}_{1}\right) \simeq \mathscr{A}_{1}, \mathcal{A}_{2} \widehat{\operatorname{CFDD}}\left(\tau_{\partial}^{-1}(-Y)\right) .
$$

Recalling that $\tau_{\partial}^{-1}(-Y)=-\left(\tau_{\partial}(Y)\right)$, this is just (1.9) with $Y$ replaced by $\tau_{\partial}(Y)$.

Proof of Theorem 6. Eq. (1.10) is equivalent to the statement that

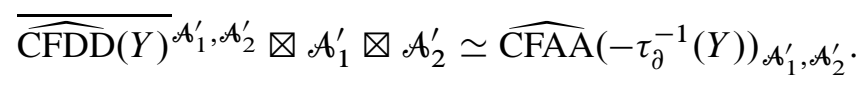

Fix a Heegaard diagram ${ }^{\alpha} \mathscr{H}^{\alpha}$ for $Y$ with $\partial_{L} \mathscr{H}=Z_{1}$ and $\partial_{R} \mathscr{H}=Z_{2}$. The pairing theorem and Propositions 3.14 and 4.1 identify the left hand side of (5.9) 
with $\widehat{\operatorname{CFAA}}\left({ }^{\alpha} \mathrm{AZ}\left(-\mathcal{Z}_{1}\right)^{\beta}{ }_{\partial_{R}} \cup_{\partial_{L}}{ }^{\beta} \overline{\mathcal{H}}^{\beta}{ }_{\partial_{R}} \cup_{\partial_{L}}{ }^{\beta} \mathrm{AZ}\left(-\mathcal{Z}_{2}\right)^{\alpha}\right)$. On the other hand, by Lemma 4.6 and Corollary 4.5, ${ }^{\alpha} \mathrm{AZ}\left(-\mathcal{Z}_{1}\right)^{\beta}{ }_{\partial_{R}} \cup_{\partial_{L}}{ }^{\beta} \overline{\mathcal{H}}^{\beta}{ }_{\partial_{R}} \cup_{\partial_{L}}{ }^{\beta} \mathrm{AZ}\left(-Z_{2}\right)^{\alpha}$ is a bordered Heegaard diagram for $\tau_{\partial}(-Y)=-\tau_{\partial}^{-1}(Y)$. This proves (5.9).

For (1.11), tensor both sides of (1.10) with $\widehat{\mathrm{CFDD}}\left(\mathbb{I}_{Z_{1}}\right) \otimes \widehat{\mathrm{CFDD}}\left(\mathbb{I}_{Z_{2}}\right)$ to get

$$
\begin{aligned}
& \operatorname{Mor}^{\mathscr{A}_{1}^{\prime} \otimes \mathcal{A}_{2}^{\prime}}\left(\mathcal{A}_{1}^{\prime}, \mathcal{A}_{2}^{\prime} \widehat{\operatorname{CFDD}}(Y),{ }^{\mathcal{A}_{1}^{\prime} \otimes \mathcal{A}_{2}^{\prime}, \mathcal{A}_{1} \otimes \mathcal{A}_{2}}\left(\widehat{\operatorname{CFDD}}\left(\mathbb{I}_{Z_{1}}\right) \otimes \widehat{\operatorname{CFDD}}\left(\mathbb{I}_{Z_{2}}\right)\right)\right) \\
& \simeq \widehat{\operatorname{CFDD}}\left(-\tau_{\partial}^{-1}(Y)\right) .
\end{aligned}
$$

Since $\cdot \bigotimes_{\mathcal{A}_{1}^{\prime} \otimes \mathcal{A}_{2}^{\prime}} \widehat{\mathrm{CFAA}}\left(\mathbb{I}_{\mathcal{Z}_{1}}\right) \otimes \widehat{\mathrm{CFAA}}\left(\mathbb{I}_{\mathcal{Z}_{2}}\right)$ is an equivalence of categories, we have

$$
\operatorname{Mor}_{\mathscr{A}_{1} \otimes \mathcal{A}_{2}}\left(\widehat{\mathrm{CFAA}}(Y)_{\mathcal{A}_{1}, \mathcal{A}_{2}}, \mathfrak{A}_{1}^{\prime} \otimes \mathcal{A}_{2}^{\prime}[\mathbb{I}]_{\mathcal{A}_{1} \otimes \mathcal{A}_{2}}\right) \simeq \mathscr{A}_{1}^{\prime} \otimes \mathcal{A}_{2}^{\prime} \widehat{\operatorname{CFDD}}\left(-\tau_{\partial}^{-1}(Y)\right) \text {. }
$$

Lowering indices gives (1.11).

Alternatively, we can apply Theorem 9 to both sides of (1.10). Specifically, viewing right actions by $A$ as left actions by $A^{\text {op }}$, rewrite (1.10) as

$$
\operatorname{Mor}_{\mathcal{A}_{1} \otimes \mathcal{A}_{2}}\left(\widehat{\operatorname{CFDD}}(Y, \mathfrak{s})_{\mathcal{A}_{1}, \mathcal{A}_{2}}, \mathcal{A}_{1} \otimes \mathcal{A}_{2}\right) \simeq \mathscr{A}_{1}, \mathcal{A}_{2} \widehat{\operatorname{CFAA}}\left(-\tau_{\partial}^{-1}(Y),-\mathfrak{s}\right) .
$$

(For extra precision, we have added the $\operatorname{spin}^{c}$ structure to the equation.) Now, by Theorem 9, the left hand side is

$$
\operatorname{Mor}_{\mathcal{A}_{1} \otimes \mathcal{A}_{2}}\left(\widehat{\operatorname{CFAA}}\left(\tau_{\partial}(Y)_{\mathcal{A}_{1}, \mathcal{A}_{2}}, \overline{\mathfrak{s}}\right), \mathcal{A}_{1} \otimes \mathcal{A}_{2}\right),
$$

while the right hand side is

$$
\mathcal{A}_{1}, \mathcal{A}_{2} \widehat{\operatorname{CFDD}}\left(\tau_{\partial}^{-1}\left(-\tau_{\partial}^{-1}(Y)\right),-\overline{\mathfrak{s}}\right)={ }_{A_{1}, \mathcal{A}_{2}} \widehat{\operatorname{CFDD}}(-Y,-\overline{\mathfrak{s}}) .
$$

Replacing $Y$ by $\tau_{\partial}^{-1}(Y)$ and conjugating the spin ${ }^{c}$ structure gives (1.11).

Proof of Corollaries 7 and 8. Eq. (1.12) follows from (1.6) of Theorem 5 by taking $Y=Y_{1}$, tensoring both sides with $\widehat{\operatorname{CFDD}}\left(Y_{2}\right)$ over $\mathcal{A}_{1}^{\prime}$, and applying the pairing theorem. Eq. (1.13) is obtained by applying (1.10) of Theorem 6 with $Y=F\left(Z_{1}\right) \times$ $[0,1]$ and then tensoring over $\mathcal{A}_{1} \otimes \mathcal{A}_{1}^{\prime}$ with $\widehat{\operatorname{CFDD}}\left(-Y_{1}\right) \otimes \widehat{\operatorname{CFDD}}\left(Y_{2}\right)$.

Eq. (1.14) of Corollary 8 can be viewed as a special case of the first part of Corollary 7 , in which one boundary component is empty. Eq. (1.15) follows by taking $Y_{1}=M_{\psi^{-1}}=-M_{\psi}$ for the first equation and taking $Y_{1}=M_{\psi}$ and reversing the orientation on $Y_{2}$ for the second equation, in view of the fact that the action of $\psi$ on a bordered manifold $Y$ is realized by gluing $M_{\psi}$ to $Y$, see [10], Lemma 5.30.

5.4. Dualizing bimodules. So far, we have used the type $A A$ module associated to $\mathrm{AZ}(\mathcal{Z})$. We next observe that the type $D D$ module associated to $\overline{\mathrm{AZ}}(-\mathcal{Z})$ gives a finite-dimensional model for the bar resolution of $\mathcal{A}(\mathbb{Z})$.

Specifically, endow $\overline{\mathcal{A}}=\operatorname{Hom}_{\mathbb{F}_{2}}\left(\mathcal{A}, \mathbb{F}_{2}\right)$ with the structure of a type $D D$ bimodule, as follows. Let Chord (Z) denote the set of chords in Z, i.e. $\operatorname{arcs}$ in $Z \backslash\{z\}$ connecting 
points in a. Recall that to each chord $\xi \in \operatorname{Chord}(\mathcal{Z})$ there is an associated algebra element $a(\xi) \in \mathcal{A}=\mathcal{A}(\mathcal{Z})$. The map

$$
\delta^{1}: \bar{A} \longrightarrow \mathcal{A} \otimes \bar{A} \otimes \mathcal{A}
$$

is defined by

$$
\begin{aligned}
\delta^{1}(\varphi)=1 \otimes \bar{d}(\varphi) \otimes 1 & +\sum_{\xi \in \operatorname{Chord}(\mathcal{Z})} a(\xi) \otimes(a(\xi) \cdot \varphi) \otimes 1 \\
& +\sum_{\xi \in \operatorname{Chord}(\mathcal{Z})} 1 \otimes(\varphi \cdot a(\xi)) \otimes a(\xi) .
\end{aligned}
$$

Here, $\bar{d}$ denotes the differential on $\overline{\mathcal{A}}$ (the dual type $A A$ structure to the bimodule ${ }_{\mathcal{A}} \mathcal{A}_{\mathcal{A}}$, see Definition 2.12) and $a \cdot \varphi$ and $\varphi \cdot a$ denote the left and right actions of $\mathcal{A}$ on $\bar{A}$. We denote this type $D D$ bimodule ${ }^{\mathcal{A}} \mathrm{bar}^{\mathcal{A}}$. (We leave it to the reader to check that this satisfies the structure equations for a type $D D$ bimodule.)

Proposition 5.11. The DD bimodule ${ }^{\mathcal{A}} \mathrm{bar}^{\mathcal{A}}$ is isomorphic to $\widehat{\operatorname{CFDD}}(\overline{\mathrm{AZ}}(-\mathrm{Z}))$.

Proof. The Heegaard diagram $\overline{\mathrm{AZ}}(-\mathcal{Z})$ is a nice diagram (see [20]). As in Proposition 4.3 , the rectangles supported in $\overline{A Z}(-\mathcal{Z})$ correspond to differentials in $\overline{\mathcal{A}(\mathcal{Z})}$. These give the terms of the form $1 \otimes \bar{d} \varphi \otimes 1$ as in (5.10).

We must consider also rectangles which go out to the boundary. Those which go out to the $\alpha$-boundary give the terms of the form $\sum_{\xi \in \operatorname{Chord}(\mathcal{Z})} a(\xi) \otimes(a(\xi) \cdot \varphi) \otimes 1$, while those which go out to the $\beta$-boundary give the terms of the form $\sum_{\xi \in \operatorname{Chord}(\mathcal{Z})} 1 \otimes$ $(\varphi \cdot a(\xi)) \otimes a(\xi)$.

Corollary 5.12. The type DD structure ${ }^{\mathcal{A}} \mathrm{bar}^{\mathcal{A}}$ is bounded.

Proof. This follows immediately from the fact that $\overline{\mathrm{AZ}}(-\mathbf{Z})$ is an admissible diagram. (Alternately, it is not hard to give a purely algebraic argument.)

This gives a finite-dimensional model for the bar complex:

Proposition 5.13. Let $\mathcal{A}=\mathcal{A}(\mathbb{Z})$. There are homotopy equivalences

$$
\begin{aligned}
& { }^{\mathcal{A}} \mathrm{bar}^{\mathcal{A}} \simeq{ }^{\mathcal{A}} \operatorname{Bar}_{r}(\mathcal{A})^{\mathcal{A}}, \\
& { }^{\mathcal{A}} \mathrm{bar}^{\mathcal{A}} \nabla_{\mathcal{A}} \mathcal{A}_{\mathcal{A}} \simeq{ }^{\mathcal{A}}[\mathbb{I}]_{\mathcal{A}}, \\
& { }^{A} \operatorname{bar}^{\mathcal{A}} \otimes_{\mathcal{A}} \overline{\mathcal{A}}_{\mathcal{A}} \simeq \widehat{\mathrm{CFDA}}\left(\tau_{\partial}^{-1}: F(\mathcal{Z}) \longrightarrow F(\mathcal{Z})\right) \text {, } \\
& { }^{A} \overline{\operatorname{bar}}^{\mathcal{A}} \otimes_{\mathcal{A}} \mathcal{A}_{\mathcal{A}} \simeq \widehat{\operatorname{CFDA}}\left(\tau_{\partial}: F(Z) \longrightarrow F(Z)\right) \text {. }
\end{aligned}
$$

Proof. To prove (5.15) observe that

$$
\left.{ }^{A} \mathrm{bar}^{\mathcal{A}} \otimes \mathcal{A}=\widehat{\mathrm{CFDD}}\left({ }^{\alpha} \overline{\mathrm{AZ}}(-Z)\right)^{\beta}\right) \otimes \widehat{\operatorname{CFAA}}\left({ }^{\beta} \mathrm{AZ}(Z)^{\alpha}\right)
$$




$$
\begin{aligned}
& \simeq \widehat{\mathrm{CFDA}}\left({ }^{\alpha} \overline{\mathrm{AZ}}(-\mathcal{Z})^{\beta} \cup{ }^{\beta} \mathrm{AZ}(\mathcal{Z})^{\alpha}\right) \\
& \simeq \widehat{\mathrm{CFDA}}(\mathbb{I}) \\
& ={ }^{\mathcal{A}}[\mathbb{I}]_{\mathcal{A}} .
\end{aligned}
$$

The first equation follows from Propositions 5.11 and 4.1, the second follows from the pairing theorem, the third is a consequence of Propositions 4.2 and 4.4, and the last is Theorem 12, proved below (also proved in [10], Theorem 4). (Note the proof of Theorem 12 does not rely on the current proposition.)

Tensoring both sides of (5.15) with ${ }^{\mathcal{A}} \operatorname{Bar}_{r}(\mathcal{A})^{\mathcal{A}}$, and using the fact that ${ }_{\mathcal{A}} \mathcal{A}_{\mathcal{A}} \otimes$ ${ }^{\mathcal{A}} \operatorname{Bar}_{r}(\mathcal{A})^{\mathcal{A}} \simeq{ }_{\mathcal{A}}[\mathbb{I}]^{\mathcal{A}}$ (essentially the statement that the algebra is quasi-isomorphic to its bar resolution), we obtain (5.14).

Eq. (5.16) follows from the pairing theorem and Propositions 4.3, 5.11, and 4.4:

$$
\begin{aligned}
& { }^{\mathcal{A}} \mathrm{bar}^{\mathcal{A}} \nabla_{\mathcal{A}} \overline{\mathcal{A}}_{\mathcal{A}}={ }^{\mathcal{A}}\left(\widehat{\mathrm{CFDD}}\left({ }^{\alpha} \overline{\mathrm{AZ}}(-\mathcal{Z})^{\beta}\right)^{\mathcal{A}} \otimes_{\mathcal{A}} \widehat{\operatorname{CFAA}}\left({ }^{\beta} \overline{\mathrm{AZZ}}(\mathcal{Z})^{\alpha}\right)_{\mathcal{A}}\right. \\
& \simeq \widehat{\mathrm{CFDA}}\left({ }^{\alpha} \overline{\mathrm{AZ}}(-\mathcal{Z})^{\beta} \cup{ }^{\beta} \overline{\mathrm{AZ}}(\mathcal{Z})^{\alpha}\right) \\
& \simeq \widehat{\operatorname{CFDA}}\left(\tau_{\partial}^{-1}: F(Z) \longrightarrow F(Z)\right) \text {. }
\end{aligned}
$$

To prove (5.17), first observe that ${ }^{\mathcal{A}} \overline{\mathrm{bar}^{\mathcal{A}}}={ }^{\mathcal{A}} \widehat{\mathrm{CFDD}}\left({ }^{\alpha} \mathrm{AZ}(-\mathbf{Z})^{\beta}\right)^{\mathcal{A}}$; this follows from Propositions 3.26 and 5.11 (or a direct calculation). So,

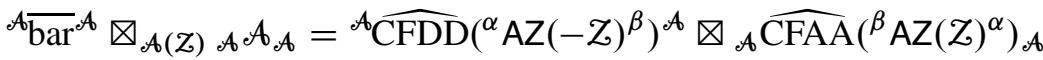

$$
\begin{aligned}
& \simeq \widehat{\mathrm{CFDA}}\left(\tau_{\partial}: F(\mathcal{Z}) \longrightarrow F(\mathcal{Z})\right) \text {. }
\end{aligned}
$$

In a similar spirit, we can use the geometry of these pieces to determine the explicit form for $\widehat{\mathrm{CFDD}}(\mathbb{I})$. See also [11] for a different argument.

Consider the type $D D$ bimodule ${ }^{\mathcal{A}(\mathcal{Z})} K^{\mathcal{A}(\mathcal{Z})}$, defined as follows. Let $\mathbf{s}$ be a subset of $\mathbf{p} / M$, and $\mathbf{t}$ denote its complement. The sets $\mathbf{s}$ and $\mathbf{t}$ have associated idempotents $I(\mathbf{s})$ and $I(\mathbf{t})$. We call such pairs $(I(\mathbf{s}), I(\mathbf{t}))$ complementary idempotents. Our type $D D$ bimodule ${ }^{\mathcal{A}(\mathcal{Z})} K^{\mathcal{A}(\mathcal{Z})}=\bigoplus_{i=-k}^{k}{ }^{\mathcal{A}(\mathcal{Z}, i)} K^{\mathcal{A}(\mathcal{Z},-i)}$ has one generator for each complementary pair of idempotents. Let

$$
\mathbf{1}=\sum_{(I, J)} I \otimes_{\mathbb{F}_{2}} J .
$$

Then the differential on ${ }^{\mathcal{A}(\mathcal{Z})} K^{\mathcal{A}(\mathcal{Z})}$ is given by

$$
\delta^{1} \mathbf{1}=\sum_{\xi \in \operatorname{Chord}(\mathcal{Z})} a(\xi) \otimes \mathbf{1} \otimes a(\xi)=\sum_{\substack{\xi \in \operatorname{Chord}(\mathcal{Z}) \\(I, J) \text { complementary }}} a(\xi) I \otimes\left(I \otimes_{\mathbb{F}_{2}} J\right) \otimes J a(\xi)
$$

As before, $a(\xi)$ denotes the algebra element in $\mathcal{A}(\mathcal{Z})$ associated to the chord $\xi$. 
Theorem 5.19 ([11], Theorem 1). The bimodule ${ }^{\mathcal{A}(\mathcal{Z})} K^{\mathcal{A}(\mathcal{Z})}$ is isomorphic to the bimodule $\widehat{\mathrm{CFDD}}\left(\mathbb{I}_{\mathcal{Z}}\right)$, with the left action of $\mathcal{A}(-\mathcal{Z})$ viewed as a right action by $\mathcal{A}(\mathcal{Z})$.

Proof. The type $D D$ identity bimodule can be represented by an $\alpha-\alpha$ bordered Heegaard diagram $\mathscr{H}$, as explained in [10], Section 5.3. Instead of directly computing this type $D D$ structure, we will compute its modulification. To this end, form $-\overline{\mathscr{H}}$, attach $\mathrm{AZ}(\mathbb{Z})$ and $\mathrm{AZ}(-\mathbf{Z})$ to its boundaries, and then destabilize the $k \alpha$-circles in $-\overline{\mathscr{H}}$. (This is the diagram illustrated on the left in Figure 7.) The type $A A$ module associated to this diagram is exactly $\widehat{\operatorname{CFDD}}(\mathbb{I})_{\mathcal{A}}(\mathcal{Z}), \mathcal{A}(-\boldsymbol{Z})$.

Observe that the simplified diagram ${ }^{\alpha} \mathrm{AZ}(\mathcal{Z})^{\beta} \cup^{\beta} \mathrm{AZ}(-\mathbb{Z})^{\alpha}$ is a nice diagram, so the associated $\widehat{\mathrm{CFAA}}$ is a bimodule with no higher action. Moreover, the bimodule structure on $\widehat{\mathrm{CFAA}}\left({ }^{\alpha} \mathrm{AZ}(\mathbb{Z})^{\beta} \cup{ }^{\beta} \mathrm{AZ}(-\mathbb{Z})^{\alpha}\right)$ (viewed as having one left and one right action) is isomorphic to the bimodule structure on ${ }_{\mathcal{A}} \mathcal{A}_{\mathcal{A}} \nabla^{\mathcal{A}^{A}} K^{\mathcal{A}} \nabla_{\mathcal{A}} \mathcal{A}_{\mathcal{A}}$ as in Proposition 4.1. So, it only remains to determine the differential on $\widehat{\operatorname{CFAA}}\left({ }^{\alpha} A Z(Z)\right)^{\beta} \cup$ $\left.{ }^{\beta} \mathrm{AZ}(-\mathcal{Z})^{\alpha}\right)$, which in turn is given by embedded rectangles. There are rectangles supported in $\left.{ }^{\alpha} A Z(Z)\right)^{\beta}$, which correspond to differentials in $\mathcal{A}(\mathcal{Z})$ (Proposition 4.2), those supported in ${ }^{\beta} \mathrm{AZ}(-\mathbb{Z})^{\alpha}$, which correspond to differentials in $\mathcal{A}(-\mathbb{Z})$, and rectangles which go between the two, which correspond to the differential $\delta^{1}$ from (5.18).

Remark 5.20. The alert reader may notice some redundancy in the proofs in this paper. In fact, it is a consequence of Lemma 8.7 that for a Koszul algebra, with dualizing bimodule ${ }^{A} K^{B}$ (which, in the present context, is $\widehat{{ }^{A}} \widehat{\mathrm{CDD}}(\mathbb{I})^{A}$, and in particular $A=B$ ), the bar resolution is homotopy equivalent to ${ }^{B} \bar{K}^{A} \otimes_{A} \bar{A}_{A} \otimes^{A} K^{B}$. But this latter bimodule is precisely the model ${ }^{\mathcal{A}} \operatorname{bar}^{\mathcal{A}}(Z)$ described in (5.10). Thus, Proposition 5.13 can be viewed as a consequence of Lemma 8.7 and Theorem 5.19.

5.5. Algebraic consequences. We start with a lemma regarding Serre functors which is probably well-known in certain circles.

Lemma 5.21. For an augmented dg algebra $\mathcal{A}$ and finite-dimensional right $\mathcal{A}_{\infty}$-modules $M_{\mathcal{A}}$ and $N_{\mathcal{A}}$ over $\mathcal{A}$,

$$
\operatorname{Mor}_{\mathcal{A}}\left(M_{\mathcal{A}}, N_{\mathcal{A}}\right)^{*} \simeq \operatorname{Mor}_{\mathcal{A}}\left(N_{\mathcal{A}}, M_{\mathcal{A}} \nabla^{\mathcal{A}} \operatorname{Bar}_{r}{ }^{\mathcal{A}} \nabla_{\mathcal{A}} \overline{\mathcal{A}}_{\mathcal{A}}\right)
$$

in a natural way. That is, the Serre functor on $\operatorname{Mod}_{\mathcal{A}}$ is given by tensoring on the right with $\operatorname{Bar}_{r}(\mathcal{A}) \otimes \overline{\mathcal{A}}$.

Proof. The left hand side of (5.22) is given by

$$
\begin{aligned}
\operatorname{Mor}_{\mathcal{A}}\left(M_{\mathcal{A}}, N_{\mathcal{A}}\right)^{*} & \cong\left(N_{\mathcal{A}} \otimes^{\mathcal{A}}{\overline{\operatorname{Bar}_{r}}}^{\mathcal{A}} \otimes_{\mathcal{A}} \bar{M}\right)^{*} \\
& \cong M_{\mathcal{A}} \otimes^{\mathcal{A}} \operatorname{Bar}_{r}{ }^{\mathcal{A}} \otimes_{\mathcal{A}} \bar{N} \\
& \simeq M_{\mathcal{A}} \otimes^{\mathcal{A}} \operatorname{Bar}_{r}{ }^{\mathcal{A}} \otimes_{\mathcal{A}} \overline{\mathcal{A}}_{\mathcal{A}} \otimes^{\mathcal{A}}{\overline{\operatorname{Bar}_{r}}}^{\mathcal{A}} \otimes_{\mathcal{A}} \bar{N}
\end{aligned}
$$




$$
\cong \operatorname{Mor}_{\mathcal{A}}\left(N_{\mathcal{A}}, M_{\mathcal{A}} \nabla^{\mathscr{A}} \operatorname{Bar}_{r}{ }^{\mathcal{A}} \nabla_{\mathcal{A}} \overline{\mathcal{A}}_{\mathcal{A}}\right),
$$

where we have used Proposition 2.10 (twice) and a dualized version of (2.11), $\bar{A} \nabla^{\mathcal{A}}{\overline{\mathrm{Bar}_{r}}}^{\mathcal{A}} \simeq{ }_{\mathcal{A}}[\mathbb{I}]^{\mathcal{A}}$.

Proof of Theorem 10. This is Lemma 5.21 plus the observation from Proposition 5.13 that

$$
\begin{aligned}
& { }^{\mathcal{A}} \mathrm{Bar}_{r}{ }^{\mathcal{A}} \nabla_{\mathcal{A}} \overline{\mathcal{A}}_{\mathcal{A}} \simeq{ }^{\mathcal{A}} \mathrm{bar}^{\mathcal{A}} \nabla_{\mathcal{A}} \overline{\mathcal{A}}_{\mathcal{A}} \\
& \simeq \widehat{\mathrm{CFDA}}\left(\tau_{\partial}^{-1}: F(\mathcal{Z}) \longrightarrow F(\mathcal{Z})\right) \text {. }
\end{aligned}
$$

Given two bimodules, we can also consider the complex of $\mathcal{A}_{\infty}$-bimodule morphisms between them. The homology of this complex, $H_{*}\left(\operatorname{Mor}_{\mathcal{A}, \mathcal{A}}\left(\mathcal{A}_{\mathcal{A}} M_{\mathcal{A}},{ }_{\mathcal{A}} N_{\mathcal{A}}\right)\right.$ ), is also called the Hochschild cohomology of $M$ with $N$ and denoted $\mathrm{HH}^{*}(M, N)$. The special case that $M=\mathcal{A}$ gives the Hochschild cohomology of $N$, the derived functor associated to the functor of invariants in $N$. With these observations, we are now ready to prove Corollary 11 .

Proof of Corollary 11. The Hochschild cohomology in question is the homology of the complex

$$
\begin{aligned}
& \operatorname{Mor}_{\mathcal{A}, \mathcal{A}^{\prime}}(\mathcal{A}, \mathcal{A} \otimes \widehat{\operatorname{CFDA}}(Y)) \\
& =\left({ }^{\mathcal{A}} \overline{\mathrm{Bar}}^{\mathcal{A}} \otimes \overline{\mathcal{A}} \otimes{ }^{\mathcal{A}} \overline{\mathrm{Bar}}^{\mathcal{A}}\right) \bigotimes_{\mathcal{A} \otimes \mathcal{A}^{\prime}}(\mathcal{A} \otimes \widehat{\mathrm{CFDA}}(Y)) \\
& \simeq\left({ }^{\mathcal{A}} \mathrm{Bar}_{r}{ }^{\mathcal{A}} \otimes \mathcal{A} \otimes{ }^{\mathcal{A}} \overline{\mathrm{Bar}}_{r}{ }^{\mathcal{A}}\right) \otimes_{\mathcal{A} \otimes \mathcal{A}^{\prime}}(\mathcal{A} \otimes \widehat{\mathrm{CFDA}}(Y)) \text {, }
\end{aligned}
$$

using Proposition 2.10, a dual version of (2.11), and (2.11) itself. Rearranging the tensor products, we obtain

$$
\begin{aligned}
& \operatorname{Mor}_{\mathcal{A}, \mathcal{A}^{\prime}}(\mathcal{A}, \mathcal{A} \otimes \widehat{\operatorname{CFDA}}(Y)) \\
& \simeq\left({ }^{\mathcal{A}} \mathrm{Bar}_{r}{ }^{\mathcal{A}}\right) \bigotimes_{\mathcal{A} \otimes \mathcal{A}^{\prime}}\left(\mathcal{A} \otimes{ }^{\mathcal{A}}{\overline{\mathrm{Bar}_{r}}}^{\mathcal{A}} \otimes \mathcal{A} \otimes \widehat{\mathrm{CFDA}}(Y)\right) \\
& =\mathrm{HC}_{*}\left(\mathcal{A} \otimes{ }^{\mathcal{A}} \overline{\mathrm{Bar}}_{r}{ }^{\mathcal{A}} \otimes \mathcal{A} \otimes \widehat{\mathrm{CFDA}}(Y)\right) \\
& \simeq \mathrm{HC}_{*}\left(\mathcal{A} \otimes \widehat{\operatorname{CFDA}}\left(\tau_{\partial}\right) \otimes \widehat{\mathrm{CFDA}}(Y)\right) \\
& \simeq \mathrm{HC}_{*}\left(\mathcal{A} \otimes \widehat{\mathrm{CFDA}}\left(\tau_{\partial}(Y)\right)\right) \\
& \simeq \widehat{\mathrm{CFK}}\left(\left(\tau_{\partial}(Y)\right)^{\circ}, K\right) \text {. }
\end{aligned}
$$

Here, $\mathrm{HC}_{*}$ denotes the Hochschild chain complex (whose homology is Hochschild homology). The second line is the definition of $\mathrm{HC}_{*}$, the third uses Proposition 5.13, the fourth uses the pairing theorem, and the last line uses [10], Theorem 14. Taking homology gives the result.

Finally, we give a simple proof that $\widehat{\mathrm{CFDA}}(\mathbb{I}) \simeq{ }^{\mathcal{A}}[\mathbb{I}]_{\mathcal{A}}$. 
Proof of Theorem 12. After raising the left index, we want to show $\widehat{\mathrm{CFDA}}\left(\mathbb{I}_{\mathcal{Z}}\right)=$ ${ }_{\mathcal{A}(\mathcal{Z})}[\mathbb{I}]_{\mathcal{A}(\mathcal{Z})}$. By the pairing theorem,

$$
\left.\widehat{\operatorname{CFAA}}\left({ }^{\beta} \mathrm{AZ}(Z)^{\alpha}\right) \otimes \widehat{\operatorname{CFDA}}\left(\mathbb{I}_{Z}\right) \simeq \widehat{\operatorname{CFAA}}\left({ }^{\beta} \mathrm{AZ}(Z)\right)^{\alpha}\right) .
$$

By Proposition 4.1, this says that

$$
\mathcal{A}(Z) \otimes \widehat{\operatorname{CFDA}}\left(\mathbb{I}_{Z}\right) \simeq \mathcal{A}(Z) .
$$

But this implies $\widehat{\mathrm{CFDA}}\left(\mathbb{I}_{\mathcal{Z}}\right) \simeq{ }^{\mathcal{A}(Z)}[\mathbb{I}]_{\mathcal{A}(Z)}$, as desired.

Remark 5.23. For the proof of Theorem 12 , it is irrelevant what $A Z(Z)$ represents geometrically. All we need to know is that $\mathcal{A}(\mathcal{Z})$ is the bordered invariant of some Heegaard diagram. We also did not use any of the other theorems in this paper, many of which depend on the invertibility of $\widehat{C F D D}(\mathbb{I})$, which itself is equivalent, via the pairing theorem, to Theorem 12 .

\section{Gradings}

Bordered Heegaard Floer homology can be equipped with gradings, and the pairing theorems described here are compatible with these gradings in a natural way. We review these notions briefly. For more details, see [9], Chapter 10 and [10], Section 2.5.

Given a pointed matched circle $\mathcal{Z}$, there is a certain Heisenberg group $G(\mathcal{Z})$ equipped with a distinguished central element $\lambda$, which has the property that $\mathcal{A}(\mathcal{Z})$ is graded by $G=G(\mathcal{Z})$. It makes sense to talk about the category of differential graded modules over this algebra. Objects in this category consist of pairs $(S, M)$, where $S$ is a $G$-set, and $M$ is a module graded by $S$ in a way which is compatible with the $G$-grading on $\mathcal{A}$.

Given $G$-sets $S$ and $T$, we can form the space $\operatorname{Hom}(S, T)$, which is orbit space of $S \times T$, divided out by its diagonal $G$ action. (Note that this is not the same as the collection of $G$-set maps $S \rightarrow T$.) Now, given differential graded modules $(S, M)$ and $(T, N)$, the morphism complex $\operatorname{Mor}((S, M),(T, N))$ is a $\mathbb{Z}$-set graded chain complex, where the grading set is $\operatorname{Hom}(S, T)$, and the underlying chain complex is as described earlier. Note that $\operatorname{Hom}(S, T)$ still admits an action by $\mathbb{Z}$ (generated by the action of $\lambda$ on $T$ or $\lambda^{-1}$ on $S$ ). In particular, the homology of the morphism space is also graded by $\operatorname{Hom}(S, T)$. (For generalities on these matters, see [10], Section 2.5.3.)

Bordered Heegaard Floer homology modules are graded in the above sense. For example, given a Z-bordered three-manifold $Y_{1}$ and a compatible bordered Heegaard diagram $\mathscr{H}$, there is a grading set $S=S(\mathscr{H})$ with the property that $\widehat{\mathrm{CFD}}\left(Y_{1}\right)$ and $\widehat{\mathrm{CFA}}\left(Y_{1}\right)$ are $S$-graded.

A graded version of Theorem 1 (for $\widehat{\mathrm{CFD}}$ ) reads as follows. 
Theorem 14. Let $Y_{1}$ and $Y_{2}$ be bordered 3-manifolds with $\partial Y_{1}=\partial Y_{2}=F(\mathcal{Z})$. Let $S_{1}$ and $S_{2}$ denote the grading sets for $Y_{1}$ and $Y_{2}$ respectively. Then, there is an identification of the grading set for $\widehat{\mathrm{CF}}\left(-Y_{1} \cup_{\partial} Y_{2}\right)$ with the $\mathbb{Z}$-set $\operatorname{Hom}\left(S_{1}, S_{2}\right)$, in such a manner that there is a graded isomorphism

$$
\widehat{\mathrm{HF}}\left(-Y_{1} \cup_{\partial} Y_{2}\right) \cong \operatorname{Ext}_{\mathcal{A}(-Z)}\left(\widehat{\mathrm{CFD}}\left(Y_{1}\right), \widehat{\mathrm{CFD}}\left(Y_{2}\right)\right)
$$

which respects the identification of grading sets.

Theorem 14 follows from the proof of Theorem 1, with two additional observations. The first is that the grading set for $\operatorname{AZ}(\mathcal{Z})$ is naturally identified with $G$ in such a manner that Proposition 4.1 holds in its graded form (i.e. $\widehat{\operatorname{CFAA}}(\operatorname{AZ}(\mathcal{Z}))$ is isomorphic to $\mathcal{A}(Z)$ as a $G(Z)$-graded bimodule); and the second observation is that the traditional pairing theorem [10], Theorem 11, used in establishing Theorem 2 also holds in a graded form, see [10], Theorem 13).

If we keep track of $\operatorname{spin}^{c}$-structures, the isomorphism in Theorem 14 is given by

$$
\bigoplus_{\substack{\mathfrak{s} \in \operatorname{spin}^{C}\left(-Y_{1} \cup_{\partial} Y_{2}\right) \\ \mathfrak{s} \mid-Y_{1}=-\mathfrak{s}_{1}, \mathfrak{s}_{Y_{2}}=\mathfrak{s}_{2}}} \widehat{\mathrm{HF}}\left(-Y_{1} \cup_{\partial} Y_{2}, \mathfrak{s}\right) \cong \operatorname{Ext}_{\mathcal{A}(-\mathcal{Z})}\left(\widehat{\operatorname{CFD}}\left(Y_{1}, \mathfrak{s}_{1}\right), \widehat{\operatorname{CFD}}\left(Y_{2}, \mathfrak{s}_{2}\right)\right) .
$$

Similarly, a version of Theorem 2 keeping track of $\operatorname{spin}^{c}$-structures is:

$$
\begin{aligned}
& \operatorname{Mor}_{\mathcal{A}(-\mathcal{Z})}(\widehat{A}(-Z) \widehat{\mathrm{CFD}}(Y, \mathfrak{s}), \mathcal{A}(-Z)) \simeq \widehat{\mathrm{CFA}}(-Y,-\mathfrak{s})_{\mathcal{A}(-Z)}, \\
& \operatorname{Mor}_{\mathcal{A}(\mathcal{Z})}\left(\widehat{\mathrm{CFA}}(Y, \mathfrak{s})_{\mathcal{A}(\mathcal{Z})}, \mathcal{A}(\mathcal{Z})\right) \simeq \mathcal{A}_{(\mathcal{Z})} \widehat{\mathrm{CFD}}(-Y,-\mathfrak{s}) .
\end{aligned}
$$

(Compare Proposition 3.14.)

Gradings can also be added in a straightforward way for Theorem 1 for $\widehat{\mathrm{CFA}}$, and to the rest of the theorems from the introduction. In particular, the gradings in Corollary 11 are obtained from a straightforward adaptation of [10], Theorem 14.

\section{Examples}

In this section, we compute a few simple examples with the Hom pairing theorem, and compare them with the results of the original, tensor product pairing theorem.

7.1. Review of the torus algebra. For simplicity, all of our examples will have torus boundary, and we will work in the central $\operatorname{spin}^{c}$-structure, so we start by reviewing the algebra $\mathcal{A}\left(T^{2}\right)=\mathcal{A}\left(T^{2}, 0\right)$ associated to the (unique) genus 1 pointed matched circle. The algebra $\mathcal{A}\left(T^{2}\right)$ has an $\mathbb{F}_{2}$-basis with 8 elements $\iota_{0}, \iota_{1}, \rho_{1}, \rho_{2}, \rho_{3}, \rho_{12}, \rho_{23}$ and $\rho_{123}$. The elements $\iota_{0}$ and $\iota_{1}$ are orthogonal idempotents. The other relations on 
the algebra are:

$$
\begin{aligned}
& \iota_{0} \rho_{1} \iota_{1}=\rho_{1}, \quad \iota_{1} \rho_{2} \iota_{0}=\rho_{2}, \quad \iota_{0} \rho_{3} \iota_{1}=\rho_{3}, \\
& \rho_{1} \rho_{2}=\rho_{12}, \quad \rho_{2} \rho_{3}=\rho_{23}, \quad \rho_{1} \rho_{23}=\rho_{123} \text {, } \\
& \rho_{12} \rho_{3}=\rho_{123}, \quad \rho_{3} \rho_{2}=0, \quad \rho_{2} \rho_{1}=0 .
\end{aligned}
$$

(See also [9], Section 11.1.)

7.2. Hom pairing theorem for some solid tori. We start by gluing together some solid tori. Consider the standard diagrams $\mathscr{H}_{\infty}$ and $\mathscr{H}_{0}$ for the $\infty$ - and 0 -framed solid tori, shown in Figure 10 (compare [9], Section 11.2).
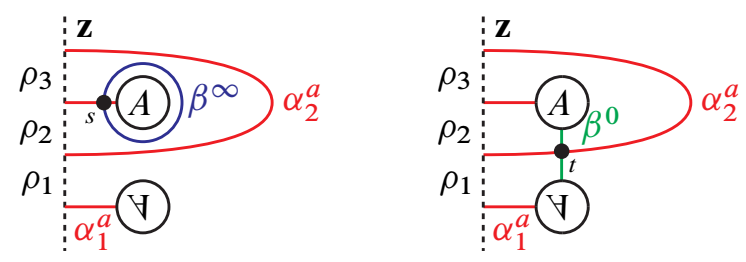

Figure 10. Standard bordered Heegaard diagrams for the $\infty$ - and 0 -framed solid tori. Left: the diagram $\mathscr{H}_{\infty}$ for the $\infty$-framed solid torus. Right: the diagram $\mathscr{H}_{0}$ for the 0 -framed solid torus.

The module $\widehat{\operatorname{CFD}}\left(\mathscr{H}_{\infty}\right)$ has a single generator $s$ with $\iota_{1} s=s$ and differential

$$
\partial(s)=\rho_{23} s .
$$

(We have used module notation for $\widehat{\mathrm{CFD}}\left(\mathscr{H}_{\infty}\right)$, so that this is another way of saying $\delta^{1}(s)=\rho_{23} \otimes s$.) The module $\widehat{\mathrm{CFD}}\left(\mathscr{H}_{0}\right)$ has a single generator $t$ with $\iota_{0} t=t$ and differential

$$
\partial(t)=\rho_{12} t .
$$

So, for instance, the chain complex $\operatorname{Mor}\left(\widehat{\mathrm{CFD}}\left(\mathscr{H}_{\infty}\right), \widehat{\mathrm{CFD}}\left(\mathscr{H}_{\infty}\right)\right)$ is generated by elements $f$ and $g$ where

$$
\begin{aligned}
& f(s)=s, \\
& g(s)=\rho_{23} s .
\end{aligned}
$$

The differential of $f$ is

$$
\begin{aligned}
(\partial f)(s) & =\partial(f(s))+f(\partial s) \\
& =\partial s+f\left(\rho_{23} s\right) \\
& =\rho_{23} s+\rho_{23} s \\
& =g(s)+g(s) \\
& =0 .
\end{aligned}
$$


Similarly, $\partial g=0$. So, $\operatorname{Ext}\left(\widehat{\operatorname{CFD}}\left(\mathscr{H}_{\infty}\right), \widehat{\operatorname{CFD}}\left(\mathscr{H}_{\infty}\right)\right)$ is two-dimensional. This is consistent with Theorem 1 , since $-\mathscr{H}_{\infty} \cup_{\partial} \mathscr{H}_{\infty}$ represents $S^{1} \times S^{2}$ and $\widehat{\mathrm{HF}}\left(S^{1} \times S^{2}\right) \cong$ $\mathbb{F}_{2} \oplus \mathbb{F}_{2}$.

It is clear from Equation (7.1) that $\operatorname{gr}(g)$ is 1 lower than $\operatorname{gr}(f)$. To illustrate the behavior of the gradings in the Hom pairing theorem, we compute this directly. We use the notation from [9], Section 11.1, for the grading groups of the torus, and the grading refinement there, taking values in a somewhat larger group $G \supset G\left(T^{2}\right)$. We have

$$
S_{D}^{\prime}\left(\mathscr{H}_{\infty}\right)=G^{\prime}\left(T^{2}\right) /\langle(-1 / 2 ; 0,-1,-1)\rangle, \quad S_{D}\left(\mathscr{H}_{\infty}\right)=G /\langle(-1 / 2 ; 0,-1)\rangle .
$$

Declaring arbitrarily that $\operatorname{gr}(s)=[(0 ; 0,0)]$, it follows that

$$
\operatorname{gr}\left(\rho_{23} s\right)=\operatorname{gr}\left(\rho_{23}\right) \operatorname{gr}(s)=[(-1 / 2 ; 0,1)] .
$$

So, inside $\left(S_{D}\left(\mathscr{H}_{\infty}\right) \times S_{D}\left(\mathscr{H}_{\infty}\right)\right) / G$,

$$
\begin{aligned}
\operatorname{gr}(f) & =[(0 ; 0,0)] \times[(0 ; 0,0)] \\
\operatorname{gr}(g) & =[(0 ; 0,0)] \times[(-1 / 2 ; 0,1)]=[(0 ; 0,0)] \times[(-1 ; 0,0)] .
\end{aligned}
$$

In particular, $\operatorname{gr}(g)=\lambda^{-1} \operatorname{gr}(f)$, as claimed.

As another simple example, $\operatorname{Mor}\left(\widehat{\mathrm{CFD}}\left(\mathscr{H}_{0}\right), \widehat{\mathrm{CFD}}\left(\mathscr{H}_{\infty}\right)\right)$ is generated by the three maps $t \mapsto \rho_{1} s, t \mapsto \rho_{3} s$, and $t \mapsto \rho_{123} s$ with differentials

$$
\begin{aligned}
& \partial\left(t \longmapsto \rho_{1} s\right)=\left(t \longmapsto \rho_{123} s\right), \\
& \partial\left(t \longmapsto \rho_{3} s\right)=\left(t \longmapsto \rho_{123} s\right) .
\end{aligned}
$$

Thus $\operatorname{Ext}\left(\widehat{\mathrm{CFD}}\left(\mathscr{H}_{0}\right), \widehat{\mathrm{CFD}}\left(\mathcal{H}_{\infty}\right)\right)$ is 1-dimensional, agreeing with $Y\left(-\mathscr{H}_{0} \cup_{\partial} \mathscr{H}_{\infty}\right)=$ $S^{3}$.

Similar computations show:

$$
\begin{aligned}
& \operatorname{Ext}\left(\widehat{\operatorname{CFD}}\left(\mathscr{H}_{0}\right), \widehat{\operatorname{CFD}}\left(\mathscr{H}_{0}\right)\right) \cong \mathbb{F}_{2} \oplus \mathbb{F}_{2}, \\
& \operatorname{Ext}\left(\widehat{\operatorname{CFD}}\left(\mathscr{H}_{\infty}\right), \widehat{\operatorname{CFD}}\left(\mathscr{H}_{0}\right)\right) \cong \mathbb{F}_{2} .
\end{aligned}
$$

7.3. $\widehat{\text { CFA }}$ is $\widehat{\text { CFD: }}$ an example. Next, we illustrate Theorem 3 for the 0 -framed solid torus $\mathscr{H}_{0}$ discussed above. The module ${ }_{\mathcal{A}\left(T^{2}\right)} \widehat{\mathrm{CFD}}\left(\mathscr{H}_{0}\right)$ has elements $t, \rho_{2} t$ and $\rho_{12} t$. The differential of $t$ is $\rho_{12} t$, so $H_{*}\left(\widehat{\operatorname{CFD}}\left(\mathcal{H}_{0}\right)\right)=\mathbb{F}_{2}\left\langle\rho_{2} t\right\rangle$. This agrees with the rank of $\widehat{\mathrm{CFA}}\left(\mathscr{H}_{0}\right)$ (on which the differential is trivial).

Moreover, we can reconstruct the $\mathcal{A}_{\infty}$-module structure on $H_{*}\left(\widehat{\mathrm{CFD}}\left(\mathscr{H}_{0}\right)\right)$. We record the $d g$ module structure on $\widehat{\mathrm{CFD}}\left(\mathscr{H}_{0}\right)$ as

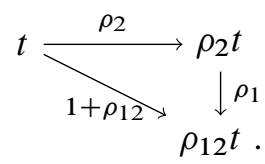


Let $x=\rho_{2} t$. Cancelling the differential from $t$ to $\rho_{12} t$ gives us $\mathcal{A}_{\infty}$-structure on $\mathbb{F}_{2}\langle x\rangle$ given by the expansion of $\rho_{2},\left(1+\rho_{12}\right)^{-1}, \rho_{1}$. That is, in $H_{*}\left(\widehat{\mathrm{CFD}}\left(\mathscr{H}_{0}\right)\right)$, $m_{3}\left(\rho_{2}, \rho_{1}, x\right)=x, m_{4}\left(\rho_{2}, \rho_{12}, \rho_{1}, x\right)=x, m_{5}\left(\rho_{2}, \rho_{12}, \rho_{12}, \rho_{1}, x\right)=x$, and so on. By contrast, the (right) $\mathscr{A}_{\infty}$-module structure on CFA $\left(\mathscr{H}_{0}\right)$ is given by $m_{3}\left(x, \rho_{3}, \rho_{2}\right)=x, m_{4}\left(x, \rho_{3}, \rho_{23}, \rho_{2}\right)=x$, and so on. Under the identification of left modules over $\mathcal{A}\left(T^{2}\right)$ with right modules over $\mathcal{A}\left(-T^{2}\right)$, these two $\mathcal{A}_{\infty}$-structures agree.

7.4. Hochschild cohomology of $\mathcal{A}\left(T^{2}\right)$. In this section, we compute the Hochschild cohomology of $\mathcal{A}\left(T^{2}\right)$; in light of Corollary 11, this is the same as computing $\widehat{\mathrm{HFK}}$ of -1 surgery on the Borromean knot.

Let II denote the identity map of the torus. Since tensoring with $\widehat{\mathrm{CFDD}}(\mathbb{I})$ gives an equivalence of categories, it is equivalent to compute

$$
\mathrm{HH}^{*}(\widehat{\mathrm{CFDD}}(\mathbb{I}), \widehat{\operatorname{CFDD}}(\mathbb{I}))=H_{*}\left(\operatorname{Mor}_{\mathcal{A}\left(T^{2}\right), \mathcal{A}^{\prime}\left(-T^{2}\right)}(\widehat{\mathrm{CFDD}}(\mathbb{I}), \widehat{\mathrm{CFDD}}(\mathbb{I}))\right),
$$

and this is what we will do.

Recall from Theorem 5.19 that ${ }^{\mathcal{A}(Z, i)} \widehat{\mathrm{CFDD}}(\mathbb{I})^{\mathcal{A}(Z,-i)}$ is generated by pairs of complementary idempotents. In the case under consideration, $Z=T^{2}$ and $i=0$, so there are two pairs of complementary idempotents $\iota_{0} \otimes \iota_{1}$ and $\iota_{1} \otimes \iota_{0}$. The differential on $\widehat{\mathrm{CFDD}}(\mathbb{I})$ is given by

$$
\begin{gathered}
\partial\left(\iota_{0} \otimes \iota_{1}\right)=\rho_{1} \otimes\left(\iota_{1} \otimes \iota_{0}\right) \otimes \rho_{1}+\rho_{3} \otimes\left(\iota_{1} \otimes \iota_{0}\right) \otimes \rho_{3} \\
+\rho_{123} \otimes\left(\iota_{1} \otimes \iota_{0}\right) \otimes \rho_{123}, \\
\partial\left(\iota_{1} \otimes \iota_{0}\right)=\rho_{2} \otimes\left(\iota_{0} \otimes \iota_{1}\right) \otimes \rho_{2} .
\end{gathered}
$$

A basis of $\operatorname{Mor}_{\mathcal{A}\left(T^{2}\right), \mathcal{A}^{\prime}\left(-T^{2}\right)}(\widehat{\mathrm{CFDD}}(\mathbb{I}), \widehat{\mathrm{CFDD}}(\mathbb{I}))$ is given by the maps $f$ sending $\iota_{i} \otimes \iota_{1-i}$ to $\rho \otimes\left(\iota_{j} \otimes \iota_{1-j}\right) \otimes \sigma$ and $\iota_{1-i} \otimes \iota_{i}$ to zero. Here $\rho$ and $\sigma$ are chords in $\mathcal{A}\left(T^{2}\right)$ respectively, with $\iota_{i} \rho \iota_{j}=\rho$ and $\iota_{1-j} \sigma \iota_{1-i}=\sigma$. Without loss of information, we will denote the map $f$ as $\langle\rho \otimes \sigma\rangle$. Then the generators of $\operatorname{Mor}_{\mathcal{A}\left(T^{2}\right), \mathcal{A}^{\prime}\left(-T^{2}\right)}(\widehat{\mathrm{CFDD}}(\mathbb{I}), \widehat{\mathrm{CFDD}}(\mathbb{I}))$ are

$$
\begin{array}{ccccc}
\left\langle\iota_{1} \otimes \iota_{0}\right\rangle, & \left\langle\rho_{23} \otimes \iota_{0}\right\rangle, & \left\langle\iota_{1} \otimes \rho_{12}\right\rangle, & \left\langle\rho_{2} \otimes \rho_{2}\right\rangle, & \left\langle\rho_{23} \otimes \rho_{12}\right\rangle, \\
\left\langle\iota_{0} \otimes \iota_{1}\right\rangle, & \left\langle\rho_{12} \otimes \iota_{1}\right\rangle, & \left\langle\iota_{0} \otimes \rho_{23}\right\rangle, & \left\langle\rho_{1} \otimes \rho_{3}\right\rangle, & \left\langle\rho_{12} \otimes \rho_{23}\right\rangle, \\
\left\langle\rho_{1} \otimes \rho_{1}\right\rangle, & \left\langle\rho_{1} \otimes \rho_{123}\right\rangle, & \left\langle\rho_{123} \otimes \rho_{1}\right\rangle, & \left\langle\rho_{123} \otimes \rho_{123}\right\rangle, & \left\langle\rho_{123} \otimes \rho_{3}\right\rangle, \\
\left\langle\rho_{3} \otimes \rho_{1}\right\rangle, & \left\langle\rho_{3} \otimes \rho_{123}\right\rangle, & \left\langle\rho_{3} \otimes \rho_{3}\right\rangle . & &
\end{array}
$$

(The maps in the first row send $\iota_{1} \otimes \iota_{0}$ to the specified element; the maps in the remaining rows send $\iota_{0} \otimes \iota_{1}$ to the specified element.)

The nontrivial differentials are given by

$$
\partial\left\langle\iota_{1} \otimes \iota_{0}\right\rangle=\left\langle\rho_{2} \otimes \rho_{2}\right\rangle+\left\langle\rho_{1} \otimes \rho_{1}\right\rangle+\left\langle\rho_{123} \otimes \rho_{123}\right\rangle+\left\langle\rho_{3} \otimes \rho_{3}\right\rangle,
$$




$$
\begin{aligned}
\partial\left\langle\rho_{23} \otimes \iota_{0}\right\rangle & =\left\langle\rho_{123} \otimes \rho_{1}\right\rangle, \\
\partial\left\langle\iota_{1} \otimes \rho_{12}\right\rangle & =\left\langle\rho_{3} \otimes \rho_{123}\right\rangle, \\
\partial\left\langle\iota_{0} \otimes \iota_{1}\right\rangle & =\left\langle\rho_{2} \otimes \rho_{2}\right\rangle+\left\langle\rho_{1} \otimes \rho_{1}\right\rangle+\left\langle\rho_{123} \otimes \rho_{123}\right\rangle+\left\langle\rho_{3} \otimes \rho_{3}\right\rangle, \\
\partial\left\langle\rho_{12} \otimes \iota_{1}\right\rangle & =\left\langle\rho_{123} \otimes \rho_{3}\right\rangle, \\
\partial\left\langle\iota_{0} \otimes \rho_{23}\right\rangle & =\left\langle\rho_{1} \otimes \rho_{123}\right\rangle, \\
\partial\left\langle\rho_{1} \otimes \rho_{3}\right\rangle & =\left\langle\rho_{12} \otimes \rho_{23}\right\rangle .
\end{aligned}
$$

A straightforward computation shows that the homology is 4-dimensional, generated by

$$
\begin{gathered}
1=\left\langle\iota_{1} \otimes \iota_{0}\right\rangle+\left\langle\iota_{0} \otimes \iota_{1}\right\rangle, \\
w=\left\langle\rho_{2} \otimes \rho_{2}\right\rangle, \quad x=\left\langle\rho_{1} \otimes \rho_{1}\right\rangle, \quad y=\left\langle\rho_{3} \otimes \rho_{3}\right\rangle, \quad z=\left\langle\rho_{123} \otimes \rho_{123}\right\rangle,
\end{gathered}
$$

with the relation

$$
w+x+y+z=0 .
$$

The element $\left\langle\iota_{1} \otimes \iota_{0}\right\rangle+\left\langle\iota_{0} \otimes \iota_{1}\right\rangle$ is the unit for the multiplication on $\mathrm{HH}^{*}(\widehat{\mathrm{CFDD}}(\mathbb{I})$, $\widehat{\mathrm{CFDD}}(\mathbb{I}))$ and all other products vanish. The grading of $\mathbf{1}$ is one lower than the grading of $w, x, y$ and $z$ (with the convention that the grading on $\mathrm{HH}^{*}$ is of cohomological type).

Remark 7.2. Recall [3] that the Hochschild cohomology of an algebra also inherits a Lie bracket, called the "Gerstenhaber bracket". Whereas the algebra structure on the Hochschild cohomology is convenient to describe in terms of automorphisms of $\widehat{\mathrm{CFDD}}(\mathbb{I})$ as above, the Gerstenhaber bracket is not transparent from this perspective. Nonetheless, with a little more work, one can identify the generators of the homology of the standard Hochschild cochain complex as

$$
\begin{aligned}
& \iota_{0}[]+\iota_{1}[], \\
& \rho_{1}\left[\rho_{1}^{*}\right]+\rho_{123}\left[\rho_{123}^{*}\right]+\rho_{12}\left[\rho_{12}^{*}\right], \\
& \rho_{3}\left[\rho_{3}^{*}\right]+\rho_{123}\left[\rho_{123}^{*}\right]+\rho_{23}\left[\rho_{23}^{*}\right], \\
& \rho_{123}\left[\rho_{3}^{*}\left|\rho_{2}^{*}\right| \rho_{1}^{*}\right] .
\end{aligned}
$$

From this, it is straightforward to verify that the Gerstenhaber bracket vanishes.

7.5. $\widehat{\text { CFAA }}$ is $\widehat{\text { CFDD }}$ with a negative boundary Dehn twist: an example. We illustrate Theorem 9 by verifying that for the standard Heegaard diagram for $\mathbb{I}_{T^{2}}$, the rank of $H_{*}\left(\widehat{\mathrm{CFDD}}\left(\mathbb{I}_{T^{2}}\right)\right)$ agrees with the rank of $H_{*}\left(\widehat{\mathrm{CFAA}}\left(\tau_{\partial}\right)\right)$. Of course, the 
theorem asserts much more than this: the bimodule structures agree. Even in this simple case, computing the $\mathcal{A}_{\infty}$-bimodule structure on the homology is somewhat tedious, and we will not record the details here.

From Theorem 5.19, or alternatively [10], Section 10.1, or [11], as a type $D D$

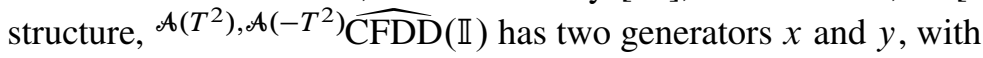

$$
\begin{aligned}
& \partial x=\left(\rho_{1} \sigma_{3}+\rho_{3} \sigma_{1}+\rho_{123} \sigma_{123}\right) \otimes y, \\
& \partial y=\left(\rho_{2} \sigma_{2}\right) \otimes x .
\end{aligned}
$$

(Here, we use $\rho$ 's to denote elements of $\mathcal{A}\left(T^{2}\right)$ and $\sigma$ 's to denote elements of $\mathcal{A}\left(-T^{2}\right)$.) Expanding this as a bimodule, $\mathcal{A}\left(T^{2}\right), \mathcal{A}\left(-T^{2}\right) \widehat{\mathrm{CFDD}}(\mathbb{I})$ has 34 generators, with differentials as shown in Figure 11. The homology is 16-dimensional.

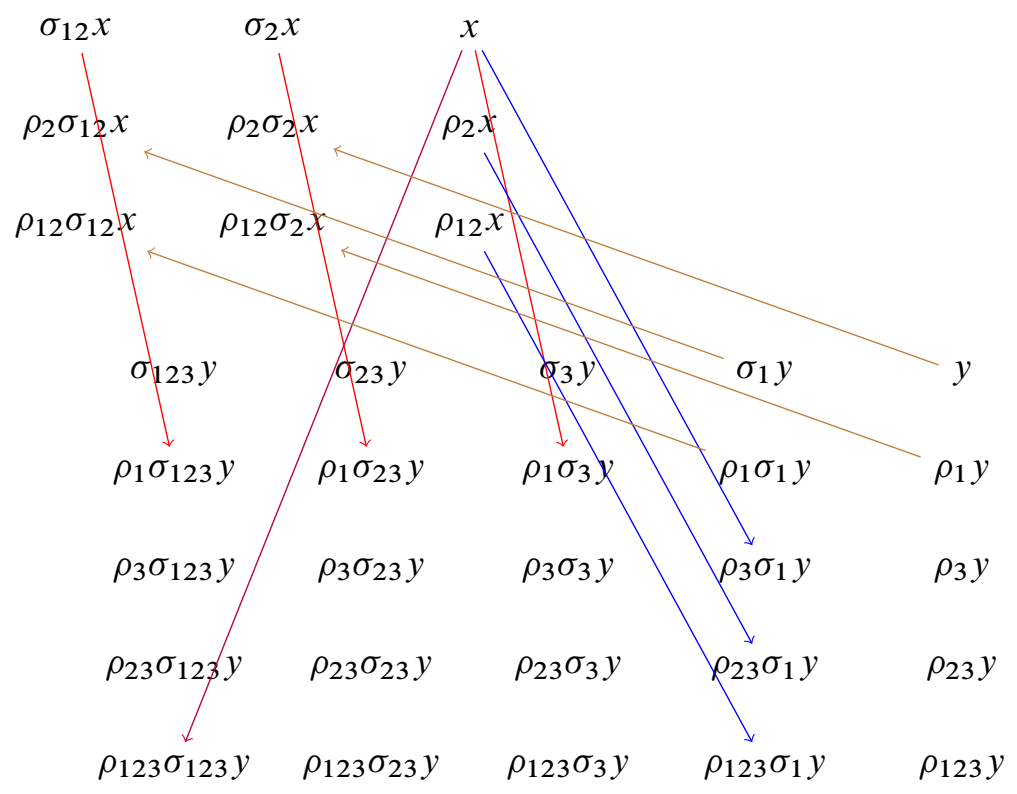

Figure 11. The $\boldsymbol{d} \boldsymbol{g}$ bimodule for $\widehat{\mathbf{C F D D}}(\mathbb{I})$. Arrows coming from the same term in $\partial x$ or $\partial y$ are parallel.

Figure 12 shows part of the standard Heegaard diagram for the negative boundary Dehn twist. Inspecting the diagram, we see that there are 16 generators in the middle $\operatorname{spin}^{c}$ structure and no provincial domains, hence no differential on $\widehat{\mathrm{CFAA}}$. Thus, the rank of $\widehat{\mathrm{CFAA}}\left(\tau_{\partial}\right)$ agrees with the rank of $H_{*}(\widehat{\mathrm{CFDD}}(\mathbb{I}))$, as claimed. 

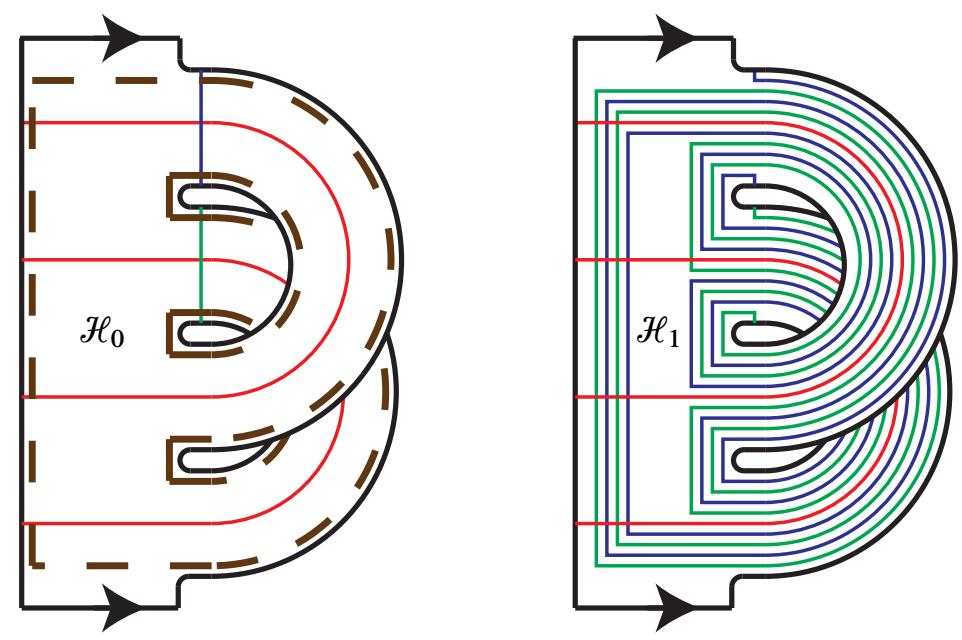

Figure 12. Standard Heegaard diagram for the negative boundary Dehn twist. On the left is half of a Heegaard diagram for the identity homeomorphism; if this surface is $\mathscr{H}_{0}$, then the result $\mathscr{H}_{0} \partial_{R} \cup_{\partial_{L}}-\mathscr{H}_{0}$ of gluing $\mathscr{H}_{0}$ to its mirror image (along $\partial_{R}$ and $\partial_{L}$, respectively) is a Heegaard diagram for the identity map. Applying a negative Dehn twist to the $\beta$ curves around the dashed curve (and isotoping away a bigon) gives the diagram $\mathscr{H}_{1}$ on the right. The result $\mathscr{H}_{1} \partial_{R} \cup_{\partial_{L}}-\mathscr{H}_{0}$ of gluing $\mathscr{H}_{1}$ to the mirror of $\mathscr{H}_{0}$ gives the standard Heegaard diagram for the positive boundary Dehn twist.

7.6. A surgery on the trefoil. We conclude by computing $\widehat{\mathrm{HF}}$ of the three-manifold $Y$ obtained as -2 Dehn filling of the left-handed trefoil complement, using Theorem 1. It follows from [9], Theorem A.11, that the type $D$ structure $\widehat{\mathrm{CFD}}(T)$ associated to the -2-framed trefoil complement is:

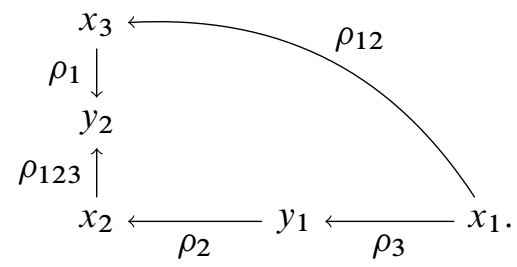

(compare [9], Figure 11.15). Here, the $x_{i}$ are generators with $\iota_{0} x_{i}=x_{i}$ and the $y_{i}$ are generators with $\iota_{1} y_{i}=y_{i}$. The arrow from $x_{1}$ to $y_{1}$, say, denotes the fact that $\rho_{3} y_{1}$ occurs in $\partial\left(x_{1}\right)$.

We compute $\operatorname{Mor}\left(\widehat{\mathrm{CFD}}\left(\mathscr{H}_{0}\right), \widehat{\mathrm{CFD}}(T)\right)$. Each $x_{i}$ is replaced by the maps $t \mapsto x_{i}$ and $t \mapsto \rho_{12} x_{i}$. Each $y_{i}$ is replaced by the three maps $t \mapsto \rho_{1} y_{i}, t \mapsto \rho_{3} y_{i}$, and 
$t \mapsto \rho_{123} y_{i}$. The differentials are:

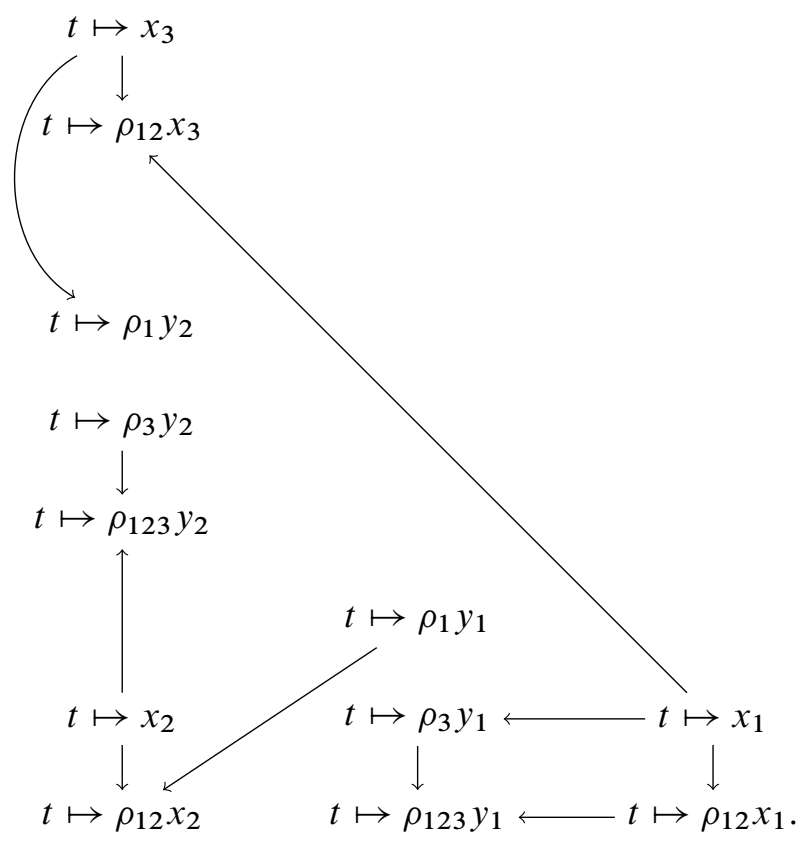

The homology $\operatorname{Ext}\left(\widehat{\mathrm{CFD}}\left(\mathcal{H}_{0}\right), \widehat{\mathrm{CFD}}(T)\right)$ is 2-dimensional, generated by $t \mapsto \rho_{1} y_{2}$ and $t \mapsto\left(\rho_{1} y_{1}+x_{2}+\rho_{3} y_{2}\right)$.

In this example, after making a choice, the grading set is identified with a double coset space of $G=G(T)$. Projecting onto the homological component of $G$, the double coset space maps to $H_{1}(Y) \cong \mathbb{Z} / 2 \mathbb{Z}$. The gradings of our two generators project to the two different elements, showing that $\widehat{\mathrm{HF}}(Y ; \mathfrak{s})$ is $\mathbb{Z} / 2 \mathbb{Z}$ for each of the two $\operatorname{spin}^{c}$ structures $\mathfrak{s}$.

This result can be compared with results from [14] or [18], which give alternate methods for computing the Heegaard Floer homology groups of -2-surgery on the left-handed trefoil.

The reader might find it disappointing that these calculations do not distinguish -2 surgery on the trefoil from that on the unknot: we gave an example which is an $L$-space. It is easy to distinguish these two three-manifolds, however, via the $\mathbb{Q}$ grading on Heegaard Floer homology from [13]; see [8] for a discussion on how to extract this information using bordered Floer homology.

\section{Relation to Koszul duality}

We will now justify the reference to Koszul duality in the $D D$ bimodule described in Equation (5.18). We also use Koszul duality to explain a symmetry in the homology 
of the algebra of $\mathcal{A}(\mathcal{Z})$ which was observed in [10]. We start with some review.

8.1. Formalities on Koszul duality. Fix a ground ring $\mathbb{k}=\bigoplus \mathbb{F}$, where $\mathbb{F}$ is a field of characteristic 2 (to avoid sign issues). In our applications, $\mathbb{F}$ is $\mathbb{F}_{2}=\mathbb{Z} / 2 \mathbb{Z}$.

Definition 8.1. A quadratic algebra $A$ over $\mathbb{k}$ is a graded algebra generated by a finitely generated $\mathbb{k}$-module $V$ of elements of degree 1, with relations $R \subset V \otimes V$ living in degree 2. Its quadratic dual algebra $A^{!}$is the algebra generated by $V^{*}$ with relations $R^{\perp} \subset(V \otimes V)^{*} \cong V^{*} \otimes V^{*}$.

Here the last isomorphism switches the tensor factors, i.e. $(V \otimes W)^{*} \cong W^{*} \otimes V^{*}$.

Definition 8.2. For augmented $d g$ algebras $A, B$ over $\mathbb{k}$, an $A$ - $B$ bimodule $M$ of any type ( $D D, A A, D A$, or $A D)$ is rank one if it is isomorphic to $\mathbb{k}$ as a $\mathbb{k}-\mathbb{k}$ bimodule.

For any quadratic algebra $A$, there is a rank 1 type $D D$ bimodule ${ }^{A} K(A)^{A^{!}}$, defined by

$$
\delta^{1}(\mathbf{1})=\sum_{i} v_{i} \otimes \mathbf{1} \otimes\left(v_{i}^{*}\right),
$$

where $\mathbf{1}$ is the generator of $K(A),\left\{v_{i}\right\}$ is a basis for $V$, and $\left\{v_{i}^{*}\right\}$ is the dual basis for $V^{*}$. The fact that $A^{!}$has relations given by $R^{\perp}$ is exactly what is necessary to make this a $D D$ bimodule.

Definition 8.4. A quadratic algebra is $K o s z u l$ if ${ }^{A} K(A)^{A^{!}}$is quasi-invertible. In this case we say that $A^{!}$is the Koszul dual of $A$.

(Bimodules $K$ and $L$ over $A$ and $B$ are quasi-inverses if $K \otimes L \simeq{ }^{A}[\mathbb{I}]_{A}$ and $L \otimes K \simeq{ }^{B}[\mathbb{I}]_{B}$; if a quasi-inverse to $K$ exists, we say $K$ is quasi-invertible.)

We compare the Definition 8.4 with the standard definition of Koszul duality for quadratic algebras in Proposition 8.13 below.

Equation (8.3) is reminiscent of Equation (5.18), suggesting that we think of $\mathcal{A}(\mathcal{Z})$ as something like a "quadratic" algebra where the "linear" elements are $a(\xi)$ for $\xi \in \operatorname{Chord}(\mathcal{Z})$. Since $\mathcal{A}(\mathcal{Z})$ has both linear terms in the relations (like $a\left(\rho_{1}\right) a\left(\rho_{2}\right)=$ $a\left(\rho_{1} \uplus \rho_{2}\right)$ when $\rho_{1}$ and $\rho_{2}$ abut) and a differential (like $\left.\partial a(\rho)=a\left(\rho_{2}\right) a\left(\rho_{1}\right)+\ldots\right)$, the notion of quadratic dual has to be extended. This can be done; in particular, the dual of a linear-quadratic algebra (where the relations have linear and quadratic terms) is a quadratic-differential algebra (with quadratic relations, but a differential); see, e.g., [19], Chapter 5. The case of linear-quadratic-differential algebras, with both types of terms, does not seem to be standard, but is a fairly straightforward generalization. Unfortunately, with the easiest definitions $\mathcal{A}(\mathcal{Z}, i)$ is not quite quadratic dual to $\mathcal{A}(\mathcal{Z},-i)$, but instead to an algebra that is only homotopy equivalent to $\mathcal{A}(\mathcal{Z},-i)$. Instead of pursuing this discussion we make the following definitions. 
Definition 8.5. Let $A$ and $B$ be augmented $d g$ algebras over $\mathbb{k}$. A Koszul dualizing bimodule between $A$ and $B$ is a type $D D$ structure ${ }^{A} K^{B}$ over $A$ and $B$ which is

- quasi-invertible,

- rank 1 , and

- such that the image of $\delta^{1}$ lies in $A_{+} \otimes K \otimes B_{+}$.

Two $d g$ algebras $A$ and $B$ over $\mathbb{k}$ are Koszul dual if there is Koszul dualizing bimodule between them.

Observe that if it is quasi-invertible then the type $D D$ bimodule ${ }^{A} K^{A^{!}}$associated to a quadratic algebra satisfies the conditions of Definition 8.5. Note also that Definition 8.5 is symmetric between $A$ and $B$, by replacing ${ }^{A} K^{B}$ by its dual ${ }^{B} \bar{K}^{A}$.

Recall that a rank-one type $D A$ bimodule ${ }^{B} M_{A}$ with $\delta_{1}^{1}=0$ is the bimodule ${ }^{B}[f]_{A}$ associated to an $\mathcal{A}_{\infty}$-map $f: A \rightarrow B$, as in [10], Definition 2.2.48. The choice of $f$ is determined by a choice of generator for $M$ as a $\mathbb{k}$-module, and changes by conjugation by a unit in $\mathbb{k}$ if we change the generator. (If $\mathbb{k}=\bigoplus \mathbb{Z} / 2 \mathbb{Z}$, as is the case in bordered Floer theory, then 1 is the unique unit in $\mathbb{k}$.)

Lemma 8.6. If $f, g: A \rightarrow B$ are $\mathcal{A}_{\infty}$-homomorphisms so that ${ }^{B}[f]_{A}$ and ${ }^{B}[g]_{A}$ are homotopy equivalent type DA structures, then $f$ and $g$ induce conjugate maps on homology, i.e. there is a unit $[u] \in H_{*}(B)$ so that for all $[a] \in H_{*}(A)$,

$$
[f(a)]=[u][g(a)][u]^{-1} .
$$

Proof. By hypothesis there are maps $\varphi:{ }^{B}[f]_{A} \rightarrow{ }^{B}[g]_{A}$ and $\psi:{ }^{B}[g]_{A} \rightarrow{ }^{B}[f]_{A}$, as well as homotopies $F:{ }^{B}[f]_{A} \rightarrow{ }^{B}[f]_{A}$ from $\psi \circ \varphi$ to $\mathbb{I}_{[f]}$ and $G:{ }^{B}[g]_{A} \rightarrow{ }^{B}[g]_{A}$ from $\varphi \circ \psi$ to $\mathbb{I}_{[g]}$. The $\mathcal{A}_{\infty}$-relations give

$$
\begin{aligned}
\partial f_{1}(a) & =f_{1}(\partial a), \\
\partial g_{1}(a) & =g_{1}(\partial a), \\
\partial \varphi_{1} & =0, \\
\partial \psi_{1} & =0, \\
f_{1}(a) \varphi_{1}+\varphi_{1} g_{1}(a) & =\partial \varphi_{2}(a)+\varphi_{2}(\partial a), \\
g_{1}(a) \psi_{1}+\psi_{1} f_{1}(a) & =\partial \psi_{2}(a)+\psi_{2}(\partial a), \\
\partial F_{1} & =\varphi_{1} \psi_{1}+1, \\
\partial G_{1} & =\psi_{1} \varphi_{1}+1 .
\end{aligned}
$$

(We are abusing notation: the map $\varphi_{1}: \mathbb{k} \rightarrow B \otimes \mathbb{k}$ corresponds to an element $\varphi_{1} \in B$, and similarly for $\psi_{1}, \varphi_{2}$, and so on.) The equations on the last line imply that $\left[\psi_{1}\right]$ 
and $\left[\varphi_{1}\right]$ are inverses in $H_{*}(B)$. So, either equation on the third line implies that, if we take $a$ to be a cycle, then there is the following equation in homology:

$$
\left[f_{1}(a)\right]\left[\varphi_{1}\right]=\left[\varphi_{1}\right]\left[g_{1}(a)\right] .
$$

That is, the maps on homology induced by $f_{1}$ and $g_{1}$ differ by conjugation by $\left[\varphi_{1}\right]$.

Lemma 8.7. Let ${ }^{A} K^{B}$ be a quasi-invertible type DD bimodule. Then its quasiinverse is given by ${ }_{B} \bar{B}_{B} \otimes^{B} \bar{K}^{A} \otimes_{A} A_{A}$.

(Here ${ }^{B} \bar{K}^{A}$ denotes the dual of ${ }^{A} K^{B}$ in the sense of Definition 2.5.)

Proof. This is essentially [10], Proposition 9.2. We repeat the proof here for the reader's convenience. Let ${ }_{B} L_{A}$ be the quasi-inverse of ${ }^{A} K^{B}$. Then

$$
\begin{aligned}
{ }_{B} L_{A} & \simeq \operatorname{Mor}_{B}\left({ }_{B} B_{B},{ }_{B} L_{A}\right) \\
& \simeq \operatorname{Mor}^{A}\left({ }^{A} K^{B} \otimes_{B} B_{B},{ }^{A} K^{B} \otimes_{B} L_{A}\right) \\
& \simeq \operatorname{Mor}^{A}\left({ }^{A} K^{B} \otimes_{B} B_{B},{ }^{A} \mathbb{I}_{A}\right) \\
& \simeq{ }^{A} K^{B} \bar{\otimes}{ }_{B} B_{B} \otimes_{A} A_{A} \otimes^{A} \mathbb{I}_{A} \\
& \simeq{ }_{B} \bar{B}_{B} \otimes^{B} \bar{K}^{A} \otimes_{A} A_{A},
\end{aligned}
$$

where here the first step follows from the fact that any module is quasi-isomorphic to its cobar resolution, the second from the fact that ${ }^{A} K^{B}$ is quasi-invertible (hence ${ }^{A} K^{B} \otimes \cdot$ induces an equivalence of homotopy categories), the third from the fact that $K$ and $L$ are quasi-inverses, the fourth follows from Proposition 2.7, and the fifth is straightforward.

Lemma 8.8. Let ${ }^{A} K^{B}$ be a Koszul dualizing bimodule between $A$ and $B$. Then ${ }^{A} K^{B}$ has a rank-one quasi-inverse. Moreover the left A-module ${ }_{A} A_{A} \otimes{ }^{A} K^{B} \otimes_{B} \bar{B}$ is a projective resolution of $\mathbb{k}$, thought of as a left A-module via the augmentation $\varepsilon$.

Proof. Lemma 8.7 gives ${ }^{A} K^{B} \otimes_{B} \bar{B}_{B} \otimes^{B} \bar{K}^{A} \otimes_{A} A_{A} \simeq{ }^{A} \mathbb{I}_{A}$, which in turn ensures that ${ }^{A} K^{B} \otimes_{B} \bar{B}_{B} \otimes^{B} \bar{K}^{A} \simeq{ }^{A} \operatorname{Bar}(A)^{A}$. From this it follows that ${ }_{A} A_{A} \otimes{ }^{A} K^{B} \otimes$ ${ }_{B} \bar{B}_{B} \otimes^{B} \bar{K}^{A} \otimes_{A} \mathbb{k}$ is a projective resolution of ${ }_{A} \mathbb{k}$. Because the image of $\delta_{\bar{K}}^{1}$ lies in $B_{+} \otimes \mathbb{k} \otimes A_{+}$, and $A_{+}$acts by zero on $A_{A} \mathbb{k}$, the terms in the above differential coming from ${ }^{B} \bar{K}^{A} \otimes_{A} \mathbb{k}$ are trivial; i.e. ${ }_{A} A_{A} \otimes^{A} K^{B} \otimes_{B} \bar{B}$ is a projective resolution of ${ }_{A} \mathbb{k}$. In particular, its homology has rank one.

Similarly, another application of Lemma 8.7 gives ${ }_{B} \bar{B}_{B} \otimes^{B} \bar{K}^{A} \otimes_{A} A_{A} \otimes^{A} K^{B} \otimes$ ${ }_{B} \mathbb{k} \simeq \mathbb{k}$. Because the image of $\delta_{K}^{1}$ lies in $A_{+} \otimes \mathbb{k} \otimes B_{+}$, and $B_{+}$acts by zero on ${ }_{B} \mathbb{k}$, it follows that ${ }_{B} \bar{B}_{B} \otimes{ }^{B} \bar{K}^{A} \otimes_{A} A$ is quasi-isomorphic to ${ }_{B} \mathbb{k}$. Thus, the homology of ${ }_{B} \bar{B}_{B} \otimes^{B} \bar{K}^{A} \otimes_{A} A_{A}$ is one-dimensional, and is the desired rank one quasi-inverse to ${ }^{A} K^{B}$.

We will also use the following version of the homological perturbation lemma. 
Lemma 8.9. Let $\mathcal{A}_{\text {and }} \mathfrak{B}$ be $\mathcal{A}_{\infty}$-algebras, ${ }_{\mathcal{A}} M_{\mathcal{B}}$ an $\mathcal{A}_{\infty}$-bimodule over $\mathcal{A}_{\text {and }} \mathcal{B}$ and ${ }_{\mathcal{A}} N$ a left $\mathcal{A}_{\infty}$-module over $\mathcal{A}$. Assume that $\mathcal{A}$ and $\mathscr{B}$ are defined over ground rings $\mathbb{k}$ and $\mathbb{\square}$ respectively, which are either $\mathbb{F}_{2}$ or finite direct sums of copies of $\mathbb{F}_{2}$,

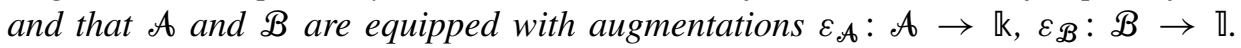
Let $f:{ }_{A} N \rightarrow{ }_{A} M$ be a quasi-isomorphism of left $\mathcal{A}_{\infty}$-modules. Then there is an $\mathcal{A}_{\infty}$-bimodule structure ${ }_{\mathcal{A}} N_{\mathcal{B}}$ on $N$, extending the given left $\mathcal{A}_{\infty}$-module structure, so that $f$ can be extended to an $\mathcal{A}_{\infty}$-bimodule quasi-isomorphism $F:{ }_{A} N_{\mathcal{B}} \rightarrow{ }_{\mathcal{A}} M_{\mathcal{B}}$.

Proof. The proof is a simple extension of standard techniques (see, for instance, [5], Section 3.3, and the references therein). In fact, we will see that the result essentially follows from the corresponding result for modules, as formulated in [11], Lemma 8.6, say.

Since is $\mathcal{A}$ defined over $\mathbb{k}$ which is a direct sum of copies of $\mathbb{F}_{2}$, any $\mathscr{A}_{\infty}$-quasiisomorphism of $\mathcal{A}_{\infty} \mathcal{A}$ modules is an $\mathcal{A}_{\infty}$-homotopy equivalence (see [10], Proposition 2.4.1, say). So, let $g:{ }_{A} M \rightarrow{ }_{A} N$ be a homotopy inverse to $f$ and let $T:{ }_{\mathcal{A}} M \rightarrow{ }_{\mathcal{A}} M$ be a homotopy between $f \circ g$ and $\mathbb{I}_{M}$.

An $\mathcal{A}_{\infty}$-bimodule structure on $N$ is a map $m^{N}: \mathcal{T}^{*} A_{+} \otimes N \otimes \mathcal{T}^{*} B_{+} \rightarrow N$ satisfying a compatibility condition. Similarly, the maps $F$ are given by a map $F: \mathcal{T}^{*}\left(A_{+}\right) \otimes N \otimes \mathcal{T}^{*}\left(B_{+}\right) \rightarrow M$ satisfying a compatibility condition. The maps $m^{N}$ and $F$ are defined by the top and bottom of Figure 13, respectively. (The map $\Delta: \mathcal{T}^{*}\left(A_{+}\right) \rightarrow \mathcal{T}_{*}\left(A_{+}\right)^{\otimes n}$ is the iterate of the obvious comultiplication on $\mathcal{T}^{*}$.)

It remains to check that the operations $m^{N}$ satisfy the $\mathcal{A}_{\infty}$-bimodule relations and that the maps $F$ satisfy the $\mathcal{A}_{\infty}$-bimodule homomorphism relations. Rather than doing this directly, we will use the bar construction to reduce to the case of modules verified in [11]. Recall that the (reduced) bar resolution of an $\mathcal{A}_{\infty}$-module ${ }_{\mathcal{A}} M$ is given by $\mathcal{T}^{*} A_{+} \otimes M$ with differential

$$
\begin{aligned}
\partial\left(a_{1} \otimes \cdots \otimes a_{n} \otimes \mathbf{x}\right) \\
\quad=\sum a_{1} \otimes \cdots \otimes \mu_{k}\left(a_{i}, \ldots, a_{i+k-1}\right) \otimes \cdots \otimes a_{n} \otimes \mathbf{x} \\
\quad+\sum a_{1} \otimes \cdots \otimes m_{k}\left(a_{n-k+2}, \ldots, a_{n}, \mathbf{x}\right) .
\end{aligned}
$$

The $\mathcal{A}_{\infty}$-relation for an $\mathcal{A}_{\infty}$-module ${ }_{\mathcal{A}} M$ is the same as the relation $\partial^{2}=0$ on the bar resolution $\mathcal{T}^{*} A_{+} \otimes M$.

Similarly, the left bar resolution of an $\mathcal{A}_{\infty}$ bimodule ${ }_{\mathcal{A}} M_{\mathcal{B}}$ is the right $\mathcal{A}_{\infty}$-module given by $\mathcal{T}_{*} A_{+} \otimes M$ with $m_{1}$ given by the formula in Equation (8.10) and higher $\mathcal{A}_{\infty}$-operations given by

$$
\begin{aligned}
& m_{1+\ell}\left(\left(a_{1} \otimes \cdots \otimes a_{n} \otimes \mathbf{x}\right), b_{1}, \ldots, b_{\ell}\right) \\
& \quad=\sum_{k=0}^{\infty} a_{1} \otimes \cdots \otimes m_{k, 1, \ell}\left(a_{n-k+1}, \ldots, a_{n}, \mathbf{x}, b_{1}, \ldots, b_{\ell}\right) .
\end{aligned}
$$

The $\mathcal{A}_{\infty}$-bimodule relations for ${ }_{\mathcal{A}} M_{\mathcal{B}}$ are equivalent to the $\mathcal{A}_{\infty}$-module relations for its left bar resolution $\mathcal{T}_{*} A_{+} \otimes M$. 

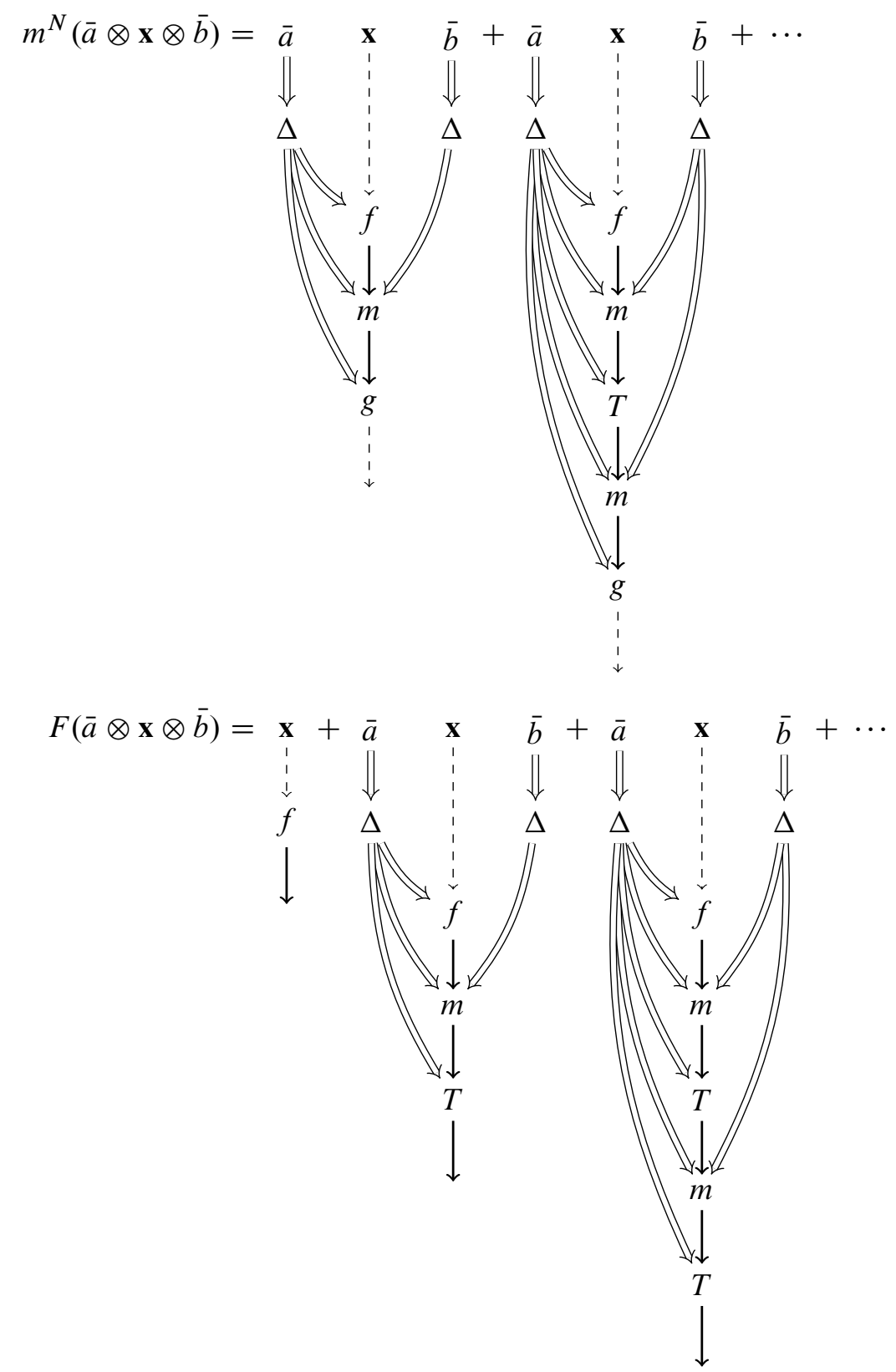

Figure 13. The induced $\mathcal{A}_{\infty}$-bimodule structure and quasi-isomorphism for Lemma 8.9. 
Turning to the case at hand, inspecting the top of Figure 13 and [11], eq. (8.7), the following two constructions give the same right $\mathcal{A}_{\infty}$-operations.

- Constructing ${ }_{\mathscr{A}} N_{\mathscr{B}}$ by the top of Figure 13 and then taking the left bar resolution.

- Taking the left bar resolution of ${ }_{\mathcal{A}} M_{\mathcal{B}}$ and the bar resolution of ${ }_{\mathcal{A}} N$ and then applying [11], eq. (8.7), to construct a right module structure on the result.

So, it follows from [11], Lemma 8.6, that the left bar resolution of ${ }_{\mathscr{A}} N_{\mathscr{B}}$ satisfies the $\mathcal{A}_{\infty}$-module relations. This proves that ${ }_{\mathcal{A}} N_{\mathscr{B}}$ satisfies the $\mathcal{A}_{\infty}$-bimodule relations.

A similar argument, comparing the bottom of Figure 13 to [11], eq. (8.9), shows that the map $F$ is an $\mathcal{A}_{\infty}$-bimodule homomorphism.

Proposition 8.11. If $A$ is any augmented $d g$ algebra and $B$ and $C$ are both Koszul dual to $A$, then $B$ and $C$ are quasi-isomorphic.

Proof. Let ${ }^{B} K^{A}$ and ${ }^{C} K^{\prime A}$ be the dualizing bimodules, and let ${ }_{A} L_{C}$ and ${ }_{A} L^{\prime}{ }_{C}$ be their respective quasi-inverses. Since ${ }^{B} K^{A}$ is right bounded, we can form the $D A$ bimodule ${ }^{B} K^{A} \otimes_{A} L^{\prime}{ }_{C}$. According to Lemma 8.8, we can use a model for ${ }_{A} L^{\prime}{ }_{C}$ which has rank one. Further, since ${ }_{A} L^{\prime} \simeq{ }_{A} \mathrm{k}$, by Lemma 8.9, ${ }_{A} L_{C}^{\prime}$ is isomorphic to a rank 1 bimodule ${ }_{A} L_{C}^{\prime \prime}$ such that $m_{i+1}\left(a_{1}, \ldots, a_{i}, l\right)=0$ for any $a_{1}, \ldots, a_{i} \in A_{+}$ and $l \in L^{\prime \prime}$. From this and the fact that the image of $\delta_{K}^{1}$ lies in $B_{+} \otimes \mathbb{k} \otimes A_{+}$, it follows that $\delta_{1}^{1}=0$ on the rank one type $D A$ bimodule ${ }^{B} K^{A} \otimes_{A} L_{C}^{\prime \prime}$.

Thus, ${ }^{B} K^{A} \otimes_{A} L_{C}^{\prime \prime}$ is the bimodule ${ }^{B}[f]_{C}$ associated to an $\mathcal{A}_{\infty}$-map $f: C \rightarrow B$. Similarly, ${ }^{C} K^{\prime A} \otimes_{A} L_{B}$ (for an appropriate model for ${ }_{A} L_{B}$ ) is the bimodule ${ }^{C}[g]_{B}$ of an $\mathcal{A}_{\infty}$-map $g: B \rightarrow C$. From the fact that $K$ and $L$ (respectively $K^{\prime}$ and $\left.L^{\prime \prime}\right)$ are quasi-inverses, it follows that ${ }^{B}[f]_{C}$ and ${ }^{C}[g]_{B}$ are quasi-inverses to each other, which in turn implies (by Lemma 8.6) that $f$ and $g$ induce isomorphisms on homology, as desired.

Recall that for an augmented $\mathcal{A}_{\infty}$-algebra $\mathcal{A}$, the cobar resolution $\operatorname{Cob}(\mathcal{A})$ is $T^{*}\left(\mathcal{A}_{+}[1]^{*}\right)$, the tensor algebra on the (shifted) dual to $\mathcal{A}_{+} \cdot \operatorname{Cob}(\mathcal{A})$ is itself a $d g$ algebra, with a product that is the tensor product in the tensor algebra and a differential that is dual to $\bar{D}^{\mathcal{A}}: T^{*}(\mathcal{A}[1]) \rightarrow T^{*}(\mathcal{A}[1])$, the operation that encodes the multiplications $\mu_{i}$ on $\mathcal{A}[10]$, Section 2.1.1.

Proposition 8.12. Any augmented dg algebra $A$ is $\operatorname{Koszul}$ dual to $\operatorname{Cob}(A)$.

Proof. The dualizing bimodule ${ }^{A} K^{\operatorname{Cob}(A)}$ is defined by

$$
\delta^{1}(\mathbf{1})=\sum_{i} a_{i} \otimes \mathbf{1} \otimes a_{i}^{*}[1],
$$

where $a_{i}$ runs over a basis of $A_{+}$and $a_{i}^{*}$ is the dual basis of $A^{*}$. Its quasi-inverse is the bimodule $\operatorname{Cob(A)} L_{A}$ defined by

$$
m_{1,1, n}\left(\left\langle b_{1}^{*}\left|b_{2}^{*}\right| \cdots \mid b_{n}^{*}\right\rangle, \mathbf{1}, a_{1}, \ldots, a_{n-1}, a_{n}\right)=b_{1}^{*}\left(a_{n}\right) b_{2}^{*}\left(a_{n-1}\right) \cdots b_{n}^{*}\left(a_{1}\right) \mathbf{1}
$$


(with all other products 0 ), where $b_{i}^{*}\left(a_{n-i}\right)$ is the canonical pairing between $b_{i}^{*} \in$ $A_{+}[1]^{*}$ and $a_{n-i} \in A$.

A Koszul dual to $A$ is, by these definitions, just a $d g$ algebra that is quasiisomorphic to $\operatorname{Cob}(A)$; compare [12]. In particular, for any augmented $d g$ algebra, $\operatorname{Cob}(\operatorname{Cob}(A))$ is quasi-isomorphic to $A$. With luck (as in the classical case of quadratic Koszul duality of Definition 8.4), there is a Koszul dual that is much smaller than $\operatorname{Cob}(A)$.

We now compare our definition of Koszul duality for quadratic algebras with the more familiar one, see for example [19], Definition 2.1.1.

Proposition 8.13. If $A$ is a Koszul, quadratic algebra in the sense of Definition 8.4, then the module ${ }_{A} A_{A} \otimes^{A} K(A)^{A^{!}} \otimes_{A} ! \overline{A^{!}}$is a graded projective resolution of $\mathbb{k}$ whose generators in homological degree $i$ are also in algebraic degree $i$.

Proof. Lemma 8.8 guarantees that ${ }_{A} A_{A} \otimes^{A} K(A)^{A^{!}} \otimes_{A^{!}} \overline{A^{!}}$is a projective resolution of $\mathrm{k}$, so we only need to check the grading property. The quadratic algebra $A$ is automatically graded, with the generators $V$ living in degree 1 . In order to extend this to a bigrading on ${ }_{A} A_{A} \otimes^{A} K(A)^{A^{!}} \otimes_{A} ! \overline{A^{!}}$it is natural to think of $A$ as bigraded, so that $V$ lives in grading $(1,0)$. (Note that the second component of this bigrading is trivial on $A$.) In order for Equation (8.3) to give a differential on ${ }^{A} K(A){ }^{A^{!}}$which changes the bigrading by $(0,-1), V^{*} \subset A^{!}$must lie in grading $(-1,-1)$. It follows that $A^{!}$lies in gradings $(-i,-i)$ for $i \geq 0$ and $\overline{A^{!}}$lies in gradings $(i, i)$, again with $i \geq 0$. This implies the proposition.

Similarly, since $\operatorname{Cob}(A) \simeq A^{!}$and $A^{!}$lies in gradings $(-i,-i)$, it follows that $\operatorname{Ext}^{i j}(\mathbb{k}, \mathbb{k})=0$ if $i \neq j$.

Remark 8.14. Definition 8.5 and Propositions 8.11 and 8.12 can be extended to the case of $\mathcal{A}_{\infty}$-algebras. The only difficulty is defining the notion of a $D D$ bimodule over two $\mathcal{A}_{\infty}$-algebras; see [10], Remark 2.2.58.

Remark 8.15. Definition 8.5 is quite similar to those considered by Lefévre-Hasegawa [6] and Keller [4], except that they work with an algebra and a coalgebra rather than two algebras. More precisely, for $C$ a coalgebra that is finite-dimensional in each grading, let $C^{*}$ be the graded dual, which is an algebra. Then a twisting cochain $\tau: C \rightarrow A$ (see, e.g., [4], Section 2.3) is the same data as a rank $1 D D$ bimodule ${ }^{A} K^{C^{*}}$ and Definition 8.5 is close to the definition of a Koszul-Moore triple in loc. cit., with some difference in the technical conditions. 
Remark 8.16. Tensoring with the dualizing bimodule ${ }^{A} K^{B}$ does not give an equivalence of categories between derived categories of modules over $A$ and over $B$, but rather between ${ }_{B}$ Mod, $\mathcal{A}_{\infty}$-modules over $B$, and ${ }_{u}^{A}$ Mod, the homotopy category of unbounded type $D$ structures. This is presumably related to the fact that the full derived categories of modules over Koszul dual algebras are not equivalent in general (see [2], Section 2.12, and [4], Section 1). In the case of the algebras considered in this paper, however, the identity bimodule ${ }^{\mathcal{A}}[\mathbb{I}]_{\mathcal{A}}$ is homotopy equivalent to a bounded module (coming from, for instance, an admissible diagram for the identity map), so the categories of bounded and unbounded type $D$ structures are quasi-equivalent, ${ }_{u}^{A} \mathrm{Mod} \simeq{ }_{b}^{A} \mathrm{Mod} \simeq{ }_{\mathcal{A}}$ Mod.

8.2. Koszul duality in bordered Floer homology. The formulation of Koszul duality in terms of bimodules is well-suited to bordered Floer homology: it allows us to use the combinatorics of Heegaard diagrams to prove the desired Koszul duality for our algebras.

Proposition 8.17. The algebra $\mathcal{A}(\mathcal{Z}, i)$ is Koszul dual to $\mathcal{A}(\mathbb{Z},-i)$.

Proof. The bimodules ${ }^{\mathcal{A}(Z, i)} \widehat{\mathrm{CFDD}}(\mathbb{I})^{\mathcal{A}(\mathcal{Z},-i)}$ and ${ }_{\mathcal{A}(\mathcal{Z},-i)} \widehat{\mathrm{CFAA}}(\mathbb{I})_{\mathcal{A}(\mathcal{Z}, i)}$, computed with respect to the standard Heegaard diagram for the identity map, obviously satisfy the conditions of Definition 8.5, so $\mathcal{A}(\mathcal{Z}, i)$ and $\mathcal{A}(\mathcal{Z},-i)$ are Koszul dual.

For the other duality, we need to consider another diagram.

Construction 8.18. Given an $\alpha$-pointed matched circle $\mathcal{Z}^{\alpha}$, the half-identity diagram $\mathcal{E}\left(Z^{\alpha}\right)$ is the $\alpha$ - $\beta$-bordered Heegaard diagram obtained as follows. Let $\Sigma_{\mathrm{dr}}$ be the disk with one-handles attached to its boundary as specified by $-\mathcal{Z}$. Let $\boldsymbol{\alpha}^{a}$ denote curves running through the one-handles, meeting the boundary along the pointed matched circle $\mathcal{Z}$. Let $\boldsymbol{\beta}^{a}$ be a collection of dual arcs: there is one in each one-handle, and $\beta_{i}^{a}$ meets only $\alpha_{i}^{a}$, transversely, and in a single point. Finally, attach another one-handle to $\Sigma_{\mathrm{dr}}$ so as to separate the $\alpha$-endpoints from the $\beta$-endpoints, to obtain a Heegaard surface $\Sigma$. The resulting $\alpha$ - $\beta$-bordered is $\mathscr{Y}\left(Z^{\alpha}\right)$.

The diagram $\mathcal{E}\left(\mathcal{Z}^{\alpha}\right)$ has two boundary components, one of which is $\mathcal{Z}^{\alpha}$ and the other of which is called the dual pointed matched circle and denoted $Z_{*}^{\beta}$. (The pointed matched circle $\mathcal{Z}_{*}^{\beta}$ naturally corresponds to turning the Morse function specifying $\mathcal{Z}$ upside-down.) Let $\mathcal{Z}_{*}^{\alpha}$, or just $\mathcal{Z}_{*}$, denote the $\alpha$-pointed matched circle twin to $\mathcal{Z}_{*}^{\beta}$.

Sometimes, we write $\mathcal{E}\left(\mathfrak{Z}^{\alpha}, \mathcal{Z}_{*}^{\beta}\right)$ to indicate both boundaries. $\mathscr{E}\left(\mathcal{Z}_{*}^{\beta}, \mathfrak{Z}^{\alpha}\right)$ is the same diagram with the roles of $\partial_{L}$ and $\partial_{R}$ switched.

For a picture of the Heegaard diagram for $\mathscr{E}\left(\mathcal{Z}^{\alpha}\right)$ for the torus, see Figure 14. The standard identity diagram is $-\mathscr{E}\left(\mathcal{Z}^{\alpha}, \mathcal{Z}_{*}^{\beta}\right){ }_{\partial_{R}} \cup_{\partial_{L}} \mathscr{E}\left(-\mathcal{Z}_{*}^{\beta},-\mathcal{Z}^{\alpha}\right)$.

Proof of Theorem 13. The first part is Proposition 8.17. 


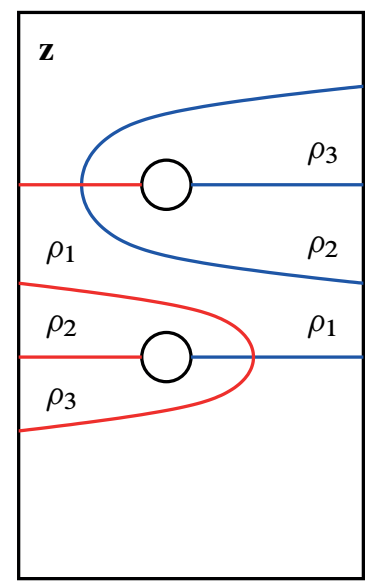

Figure 14. Heegaard diagram for $\mathcal{E}$ for a genus one surface. This is the Heegaard diagram $\mathcal{E}\left(\mathbb{Z}^{\alpha}\right)$ for the case where the genus is one, so $\mathbb{Z}=\mathbb{Z}_{*}$, as described in Construction 8.18.

The bimodules ${ }^{\mathcal{A}(\mathcal{Z}, i)} \widehat{\mathrm{CFDD}}(\mathcal{E}(\mathcal{Z}))^{\mathcal{A}\left(\mathcal{Z}_{*}, i\right)}$ and ${ }_{\mathcal{A}\left(\mathcal{Z}_{*}, i\right)} \widehat{\mathrm{CFAA}}(-\mathcal{E}(\mathcal{Z}))_{\mathcal{A}(\mathcal{Z}, i)}$ also satisfy the conditions of Definition 8.5 , so $\mathcal{A}(\mathcal{Z}, i)$ and $\mathcal{A}\left(\mathcal{Z}_{*}, i\right)$ are Koszul dual.

Proposition 8.11 now implies that $\mathcal{A}(\mathcal{Z},-i)$ is quasi-isomorphic to $\mathcal{A}\left(Z_{*}, i\right)$.

It is interesting to note that in the case of the pointed matched circle $\mathcal{Z}$ for the torus algebra with $i=0$, both bimodules $\widehat{\operatorname{CFDD}}(\mathscr{E}(\mathcal{Z}), 0)$ and $\widehat{\operatorname{CFDD}}(\mathbb{I})$ give Koszul self-dualities of the torus algebra $\mathcal{A}(\mathcal{Z}, 0)$. However, the two bimodules are different; the bimodule $\widehat{\operatorname{CFDD}}(\mathcal{E}(\mathcal{Z}), 0)$ is given by

$$
\delta(\mathbf{1})=\rho_{1} \otimes \mathbf{1} \otimes \rho_{1}+\rho_{2} \otimes \mathbf{1} \otimes \rho_{2}+\rho_{3} \otimes \mathbf{1} \otimes \rho_{3}
$$

(a fact which can be verifying by enumerating holomorphic curves in Figure 14). Contrast this with $\widehat{\mathrm{CFDD}}(\mathbb{I}, 0)$, which by Equation (7.3) is given by

$$
\delta(\mathbf{1})=\rho_{1} \otimes \mathbf{1} \otimes \rho_{1}+\rho_{2} \otimes \mathbf{1} \otimes \rho_{2}+\rho_{3} \otimes \mathbf{1} \otimes \rho_{3}+\rho_{123} \otimes \mathbf{1} \otimes \rho_{123} .
$$

If we tensor one of these bimodules with the inverse of the other, we get a non-trivial $\mathcal{A}_{\infty}$-automorphism $f: \mathcal{A}\left(T^{2}\right) \rightarrow \mathcal{A}\left(T^{2}\right)$, given by

$$
\begin{aligned}
f_{1}(x) & =x, \\
f_{3}\left(\rho_{3}, \rho_{2}, \rho_{1}\right) & =\rho_{123},
\end{aligned}
$$

with all other terms being 0 . (This automorphism can also be computed by counting holomorphic curves in Figure 14 as a $D A$ bimodule.)

In a different direction, the symmetry $H_{*}(\mathcal{A}(\mathcal{Z}, i)) \cong H_{*}\left(\mathcal{A}\left(\mathcal{Z}_{*},-i\right)\right)$ explains some numerical coincidences apparent in the homology calculations from [10].

For $\mathbb{Z}$ any pointed matched circle of genus $k>0, \mathcal{A}(\mathbb{Z},-k) \cong \mathbb{F}_{2}$ and $\mathcal{A}(\mathbb{Z}$, $-k+1$ ) have no differential, so 


$$
\begin{aligned}
\operatorname{dim}\left(H_{*}(\mathcal{A}(\mathcal{Z},-k))\right) & =\operatorname{dim}(\mathcal{A}(\mathcal{Z},-k))=1, \\
\operatorname{dim}\left(H_{*}(\mathcal{A}(\mathcal{Z},-k+1))\right) & =\operatorname{dim}(\mathcal{A}(\mathcal{Z},-k+1))=8 k^{2},
\end{aligned}
$$

both of which depend only on $k$, not the pointed matched circle. It now follows from Theorem 13 that

$$
\begin{gathered}
H_{*}(\mathcal{A}(\mathcal{Z}, k-1))=8 k^{2}, \\
H_{*}(\mathcal{A}(\mathcal{Z}, k))=1,
\end{gathered}
$$

for any genus $k$ pointed matched circle $\mathcal{Z}$, as well.

In [10], we computed that if $\mathcal{Z}$ is the split pointed matched circle of genus 2 then

$$
\sum_{i} \operatorname{dim}\left(H_{*}(\mathcal{A}(\mathcal{Z}, i))\right)=T^{-2}+32 T^{-1}+98+32 T+T^{2},
$$

while if $\mathcal{Z}$ denotes the antipodal pointed matched circle of genus 2 then

$$
\sum_{i} \operatorname{dim}\left(H_{*}(\mathcal{A}(\mathcal{Z}, i))\right)=T^{-2}+32 T^{-1}+70+32 T+T^{2} .
$$

The coincidences in the Poincare polynomials in all but the middle-most term (and their symmetry) is now explained by Koszul duality.

In general, $H_{*}(\mathcal{A}(\mathcal{Z}), i)$ is not necessarily isomorphic to $H_{*}(\mathcal{A}(\mathcal{Z}),-i)$. For instance, for the genus 3 pointed matched circle $Z_{1}$ with matched points

$$
(1,7), \quad(2,9), \quad(3,5), \quad(4,6), \quad(8,11), \quad(10,12),
$$

computer computation gives

$$
\begin{aligned}
& \sum_{i} \operatorname{dim}\left(H_{*}\left(\mathcal{A}\left(Z_{1}, i\right)\right)\right) \cdot T^{i} \\
& \quad=T^{-3}+72 \cdot T^{-2}+600 \cdot T^{-1}+1224+616 \cdot T+72 \cdot T^{2}+T^{3},
\end{aligned}
$$

and in particular $\operatorname{dim}\left(H_{*}\left(\mathcal{A}\left(\mathcal{Z}_{1}, 1\right)\right)\right) \neq \operatorname{dim}\left(H_{*}\left(\mathcal{A}\left(\mathcal{Z}_{1},-1\right)\right)\right)$. Similarly, for the dual pointed matched circle $Z_{2}=Z_{1}^{*}$, with matched points

$$
(1,10), \quad(2,4), \quad(3,12), \quad(5,11), \quad(6,8), \quad(7,9),
$$

computer computation gives

$$
\begin{aligned}
& \sum_{i} \operatorname{dim}\left(H_{*}\left(\mathcal{A}\left(\mathcal{Z}_{2}, i\right)\right)\right) \cdot T^{i} \\
& \quad=T^{-3}+72 \cdot T^{-2}+616 \cdot T^{-1}+1224+600 \cdot T+72 \cdot T^{2}+T^{3},
\end{aligned}
$$

which is consistent with Theorem 13. 
Remark 8.19. In light of Auroux's reinterpretation of bordered Floer theory [1], the referee points out that it is interesting to compare the results of this section with [21], Section (5k).

\section{A. User's guide to orientation conventions}

Type $D$ structures (Definition 2.2) are written with the algebra as a superscript, and modules with the algebra as a subscript. Examples:

${ }^{\mathcal{A}} M \quad$ Left type $D$ structure over $\mathcal{A}$.

$M_{\mathcal{A}} \quad$ Right $\mathcal{A}_{\infty}$-module over $\mathcal{A}$.

${ }^{\mathcal{A}} M_{\mathscr{B}} \quad$ Type $D A$ bimodule; left type $D$ over $\mathcal{A}$, right type $A$ over $\mathcal{B}$.

The algebras $\mathcal{A}(\mathbb{Z})$ and $\mathcal{A}(-\mathbb{Z})$ are opposites:

$$
\mathcal{A}(\mathcal{Z})^{\mathrm{op}}=\mathcal{A}(-\mathcal{Z}) \text {. }
$$

So, there are identifications $\operatorname{Mod}_{\mathcal{A}(\mathcal{Z})} \equiv_{\mathcal{A}(-\mathcal{Z})} \operatorname{Mod}$ and $\operatorname{Mod}^{\mathcal{A}(\mathcal{Z})} \equiv \mathcal{A}(-\mathcal{Z})$ Mod. With respect to these identifications,

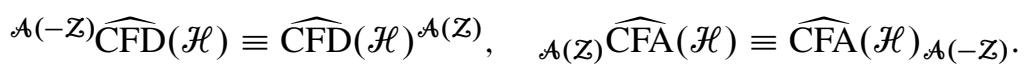

The following modules are associated to $\alpha$ - or $\beta$-bordered Heegaard diagrams with boundary $\mathcal{Z}^{\alpha}$ or $\mathcal{Z}^{\beta}$, respectively:

\begin{tabular}{lcc}
\hline Diagram & Type $D$ structures & Type $A$ structures \\
\hline $\mathscr{H}^{\alpha}$ & $\mathcal{A}(-\mathcal{Z}) \widehat{\mathrm{CFD}}(\mathscr{H}) \equiv \widehat{\mathrm{CFD}}(\mathscr{H})^{\mathcal{A}(Z)}$ & $\mathcal{A}(-\mathcal{Z}) \widehat{\mathrm{CFA}}(\mathscr{H}) \equiv \widehat{\mathrm{CFA}}(\mathscr{H})_{\mathcal{A}(Z)}$ \\
$\mathscr{H}^{\beta}$ & $\mathcal{A}(\mathcal{Z}) \widehat{\mathrm{CFD}}(\mathscr{H}) \equiv \widehat{\mathrm{CFD}}(\mathscr{H})^{\mathcal{A}(-Z)}$ & $\mathcal{A}(\mathcal{Z}) \widehat{\mathrm{CFA}}(\mathscr{H}) \equiv \widehat{\mathrm{CFA}}(\mathscr{H})_{\mathcal{A}(-Z)}$ \\
\hline
\end{tabular}

The surface associated to an orientation-reversed pointed matched circle is the orientation reverse of the surface associated to the pointed matched circle: $F(-Z)=$ $-F(Z)$.

If $\mathscr{H}^{\alpha}$ is an $\alpha$-bordered Heegaard diagram there is a corresponding $\beta$-bordered Heegaard diagram $\overline{\mathscr{H}}^{\beta}$ (Definition 3.11). The corresponding invariants are dual (Proposition 3.14):

$$
\overline{\mathcal{A}(-Z) \widehat{\mathrm{CFD}}\left(\mathscr{H}^{\alpha}\right)}=\widehat{\mathrm{CFD}}\left(\overline{\mathscr{H}}^{\beta}\right)^{\mathcal{A}(-Z)}, \quad \widehat{\widehat{\mathrm{CFA}}\left(\mathscr{H}^{\alpha}\right)_{\mathcal{A}(Z)}}={ }_{\mathcal{A}(\mathcal{Z})} \widehat{\mathrm{CFA}}\left(\overline{\mathscr{H}}^{\beta}\right) .
$$

One can also reverse the orientation of $\mathscr{H}^{\alpha}$, giving a new $\alpha$-bordered Heegaard diagram $-\mathscr{H}^{\alpha}$. Again, the invariants are dual (Proposition 3.15):

$$
\widehat{\mathcal{A}(-Z) \widehat{\mathrm{CFD}}\left(\mathscr{H}^{\alpha}\right)}=\widehat{\mathrm{CFD}}\left(-\mathscr{H}^{\alpha}\right)^{\mathcal{A}(-Z)}, \quad \widehat{\widehat{\mathrm{CFA}}\left(\mathscr{H}^{\alpha}\right)_{\mathcal{A}(Z)}}={ }_{\mathcal{A}(Z)} \widehat{\mathrm{CFA}}\left(-\mathscr{H}^{\alpha}\right) .
$$


Analogous statements hold for bimodules associated to bordered Heegaard diagrams with two boundary components (Propositions 3.25 and 3.26).

Given a strongly based mapping class $\varphi: F\left(Z_{1}\right) \rightarrow F\left(Z_{2}\right)$ there is an associated mapping cylinder $M_{\varphi}$, with $\partial_{L} M_{\varphi}=-F\left(\mathcal{Z}_{1}\right)$ and $\partial_{R} M_{\varphi}=F\left(Z_{2}\right)$; see Construction 3.21 and also [10], Section 5.3. To emphasize: maps go from (minus) the left boundary to the right boundary. There is an action of the mapping class groupoid on the set of bordered 3-manifolds, by

$$
\varphi\left(Y, \psi: F\left(\mathcal{Z}_{1}\right) \rightarrow \partial Y\right)=\left(Y, \psi \circ \varphi^{-1}: F\left(\mathcal{Z}_{2}\right) \longrightarrow \partial Y\right),
$$

where $\varphi: F\left(\mathcal{Z}_{1}\right) \rightarrow F\left(\mathcal{Z}_{2}\right)$; see Definition 3.22. Equivalently, $\varphi(Y)=Y \cup_{F\left(\mathcal{Z}_{1}\right)}$ $M_{\varphi}$.

Note that if we reverse the roles of the left and right boundary on the mapping cylinder $M_{\varphi}$, where $\varphi:-\partial_{L} M_{\varphi} \rightarrow \partial_{R} M_{\varphi}$, we get the mapping cylinder of $-\varphi^{-1}$ : $-\partial_{R} M_{\varphi} \rightarrow \partial_{L} M_{\varphi}$. In particular, switching the two sides of the mapping cylinder of a positive Dehn twist gives the mapping cylinder of another positive Dehn twist, as both taking the inverse of $\varphi$ and reversing the orientation of the surfaces switch positive and negative Dehn twists.

The strongly based mapping class $\tau_{\partial}$ plays a special role. The map $\tau_{\partial}: F(\mathcal{Z}) \rightarrow$ $F(\mathcal{Z})$ is a positive Dehn twist around the boundary of the preferred disk $D_{\alpha} \cup s \cup D_{\beta}$ in $F(Z)$. The effect of gluing $\tau_{\partial}$ to a strongly bordered 3-manifold with two boundary components is to decrease the framing on the arc by 1 .

Of particular importance in this paper is the bordered diagram $\operatorname{AZ}(\mathbb{Z})$ and its mirror $\overline{A Z}(Z)$ (Section 4). These are defined so that

$$
\partial \mathrm{AZ}(\mathcal{Z})=\mathcal{Z}^{\alpha} \amalg \mathcal{Z}^{\beta}=\partial \overline{\mathrm{AZ}}(\mathbb{Z}) .
$$

To make it clear which boundary is being glued, we often write ${ }^{\alpha} A Z(Z){ }^{\beta}$ or ${ }^{\beta} \mathrm{AZ}(\mathcal{Z})^{\alpha}$, to indicate whether we think of the $\alpha$ - of $\beta$-boundary of $\mathrm{AZ}(\mathcal{Z})$ as on the left. These are two ways of writing the same diagram. In particular,

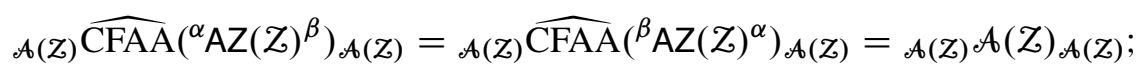

and in both cases, the $\alpha$-boundary corresponds to the right action. (The second equality uses Proposition 4.1.) If the $\alpha$ boundary corresponds to the left action then the bimodules are

$$
\begin{aligned}
& \left.\left.{ }_{\mathcal{A}(-Z)} \widehat{\mathrm{CFAA}}\left({ }^{\alpha} \mathrm{AZ}(Z)\right)^{\beta}\right)_{\mathcal{A}(-Z)}={ }_{\mathcal{A}(-Z)} \mathcal{A}(-Z)\right)_{\mathcal{A}(-Z)} \\
& ={ }_{\mathcal{A}(-Z)} \widehat{\mathrm{CFAA}}\left({ }^{\beta} \mathrm{AZ}(Z)^{\alpha}\right)_{\mathcal{A}(-Z)} \text {. }
\end{aligned}
$$

Similarly,

$$
\widehat{A}(Z) \widehat{\operatorname{CFAA}}\left({ }^{\alpha} \overline{A Z}(Z){ }^{\beta}\right)_{\mathcal{A}(Z)}={ }_{\mathcal{A}(Z)} \overline{\mathcal{A}(Z)}_{\mathcal{A}(Z)} .
$$

The following is a representative sample of the valid gluings of $A Z$ and $\overline{A Z}$ pieces, and what they represent, as maps from (minus) the left boundary to the right boundary: 


$$
\begin{aligned}
& { }^{\alpha} \mathrm{AZ}(-\mathbb{Z})^{\beta} \cup{ }^{\beta} \mathrm{AZ}(\mathbb{Z})^{\alpha}, \quad \tau_{\partial}: F\left(\mathcal{Z}^{\alpha}\right) \longrightarrow F\left(\mathbb{Z}^{\alpha}\right), \\
& { }^{\alpha} \mathrm{AZ}(\mathbb{Z})^{\beta} \cup{ }^{\beta} \mathrm{AZ}(-\mathcal{Z})^{\alpha}, \quad \tau_{\partial}: F\left(-Z^{\alpha}\right) \longrightarrow F\left(-Z^{\alpha}\right), \\
& { }^{\beta} \mathrm{AZ}(-\mathcal{Z})^{\alpha} \cup{ }^{\alpha} \mathrm{AZ}(\mathcal{Z})^{\beta}, \quad \tau_{\partial}: F\left(Z^{\beta}\right) \longrightarrow F\left(Z^{\beta}\right), \\
& { }^{\alpha} \overline{\mathrm{AZ}}(-\mathbb{Z})^{\beta} \cup{ }^{\beta} \mathrm{AZ}(\mathbb{Z})^{\alpha}, \quad \mathbb{I}: F(\mathbb{Z}) \longrightarrow F(\mathbb{Z}), \\
& { }^{\alpha} \mathrm{AZ}(-\mathbb{Z})^{\beta} \cup{ }^{\beta} \overline{\mathrm{AZ}}(\mathbb{Z})^{\alpha}, \quad \mathbb{I}: F(\mathbb{Z}) \longrightarrow F(\mathbb{Z}), \\
& { }^{\alpha} \overline{\mathrm{AZ}}(-\mathcal{Z})^{\beta} \cup{ }^{\beta} \overline{\mathrm{AZ}}(\mathbb{Z})^{\alpha}, \quad \tau_{\partial}^{-1}: F(\mathbb{Z}) \longrightarrow F(\mathcal{Z}) \text {. }
\end{aligned}
$$

See Propositions 4.2 and 4.4 and Corollary 4.5 .

\section{References}

[1] D. Auroux, Fukaya categories of symmetric products and bordered Heegaard-Floer homology. J. Gökova Geom. Topol. GGT 4 (2010), 1-54. MR 2755992

Preprint 2001. arXiv:1001.4323

[2] A. Beilinson, V. Ginzburg, and W. Soergel, Koszul duality patterns in representation theory. J. Amer. Math. Soc. 9 (1996), 473-527. Zbl 0864.17006 MR 1322847

[3] M. Gerstenhaber, The cohomology structure of an associative ring. Ann. of Math. (2) 78 (1963), 267-288. Zbl 0131.27302 MR 0161898

[4] B. Keller, Koszul duality and coderived categories (after K. Lefèvre). http://people.math.jussieu.fr/ keller/publ/kdc.dvi.

[5] B. Keller, Introduction to $A$-infinity algebras and modules. Homology Homotopy Appl. 3 (2001), 1-35. Zbl 0989.18009 MR 1854636 Preprint 1999. arXiv:math.RA/9910179

[6] K. Lefèvre-Hasegawa, Sur les $A_{\infty}$-catégories. Ph.D. thesis. Université Denis DiderotParis 7, 2003. arXiv:math.CT/0310337

[7] R. Lipshitz, P. S. Ozsváth, and D. P. Thurston, Computing cobordism maps with bordered Floer homology. In preparation.

[8] R. Lipshitz, P. S. Ozsváth, and D. P. Thurston, Relative $\mathbb{Q}$-gradings from bordered Floer theory. In preparation.

[9] R. Lipshitz, P. S. Ozsváth, and D. P. Thurston, Bordered Heegaard Floer homology: Invariance and pairing. Preprint 2008. arXiv:0810.0687v4

[10] R. Lipshitz, P. S. Ozsváth, and D. P. Thurston, Bimodules in bordered Heegaard Floer homology. Preprint 2010. arXiv:1003.0598v3

[11] R. Lipshitz, P. S. Ozsváth, and D. P. Thurston, Computing $\widehat{\mathrm{HF}}$ by factoring mapping classes. Preprint 2010. arXiv:1010.2550v3

[12] D. M. Lu, J. H. Palmieri, Q.-S. Wu, and J. J. Zhang, Koszul equivalences in $A_{\infty}$-algebras. New York J. Math. 14 (2008), 325-378. MR 2430869 Zbl 1191.16011

Preprint 2001. arXiv:0710.5492v1 
[13] P. S. Ozsváth and Z. Szabó, Absolutely graded Floer homologies and intersection forms for four-manifolds with boundary. Adv. Math. 173 (2003), 179-261. MR 1957829 Zbl 1025.57016 Preprint 2001. arXiv:math.SG/0110170

[14] P. S. Ozsváth and Z. Szabó, On the Floer homology of plumbed three-manifolds. Geom. Topol. 7 (2003), 185-224. MR 1988284 Zbl 1130.57302

Preprint 2002. arXiv:math.SG/0203265

[15] P. S. Ozsváth and Z. Szabó, Holomorphic disks and topological invariants for closed threemanifolds. Ann. of Math. (2) 159 (2004), 1027-1158. MR 2113019 Zbl 1073.57009 Preprint 2001. arXiv:math.SG/0105202

[16] P. S. Ozsváth and Z. Szabó, Holomorphic disks and three-manifold invariants: properties and applications. Ann. of Math. (2) 159 (2004), 1159-1245. MR 2113020Zbl 1081.57013 Preprint 2001. arXiv:math.SG/0101206

[17] P. S. Ozsváth and Z. Szabó, Holomorphic triangles and invariants for smooth fourmanifolds. Adv. Math. 202 (2006), 326-400. MR 2222356 Zbl 1099.53058

Preprint 2001. arXiv:math.SG/0110169

[18] P. S. Ozsváth and Z. Szabó, Knot Floer homology and integer surgeries. Algebr. Geom. Topol. 8 (2008), 101-153. MR 2377279 Zbl 1181.57018

[19] A. Polishchuk and L. Positselski, Quadratic Algebras. Amer. Math. Soc., Providence (RI), 2005. MR 2177131 Zbl 1145.16009

[20] S. Sarkar and J. Wang, An algorithm for computing some Heegaard Floer homologies. Ann. of Math. (2) 171 (2010), 1213-1236. MR 2630063 Zbl 05712751

Preprint 2006. arXiv:math/0607777

[21] P. Seidel, Fukaya categories and Picard-Lefschetz theory. European Mathematical Society, Zürich 2008. MR 2441780 Zbl 1159.53001

[22] R. Zarev, Bordered Floer homology for sutured manifolds.

Preprint 2009. arXiv:0908.1106

[23] R. Zarev, Joining and gluing sutured Floer homology. Preprint 2010. arXiv:1010.3496

Received May 24, 2010

Department of Mathematics, Columbia University, 2990 Broadway, New York, NY 10027, U.S.A

E-mail: lipshitz@math.columbia.edu

Department of Mathematics, Columbia University, 2990 Broadway, New York, NY 10027, U.S.A.

E-mail: petero@math.columbia.edu

Department of Mathematics, Barnard College, Columbia University, 3009 Broadway,

New York, NY 10027-6598, U.S.A.

E-mail: dthurston@barnard.edu 